\title{
Intrinsic First and Higher-Order Topological Superconductivity in a Doped Topological Insulator
}

Harley Scammell ( $\square$ h.scammell@unsw.edu.au )

The University of New South Wales

Julian Ingham

Boston University

Max Geier

Freie Universitat Berlin https://orcid.org/0000-0002-7552-5763

\section{Tommy Li}

Freie Universitat Berlin

\section{Article}

Keywords: topological, superconductivity, diagonalisation, heterostructure

Posted Date: September 27th, 2021

DOl: https://doi.org/10.21203/rs.3.rs-899707/v1

License: (c) (i) This work is licensed under a Creative Commons Attribution 4.0 International License. Read Full License 


\title{
Intrinsic first and higher-order topological superconductivity in a doped topological insulator
}

\author{
Harley D. Scammell, ${ }^{1,2, *}$ Julian Ingham, ${ }^{3}$ Max Geier, ${ }^{4,5}$ and Tommy $\mathrm{Li}^{4}$ \\ ${ }^{1}$ School of Physics, University of New South Wales, Sydney 2052, Australia \\ ${ }^{2}$ Australian Research Council Centre of Excellence in Future Low-Energy \\ Electronics Technologies, University of New South Wales, Sydney 2052, Australia \\ ${ }^{3}$ Physics Department, Boston University, Commonwealth Avenue, Boston, MA 02215, USA \\ ${ }^{4}$ Dahlem Center for Complex Quantum Systems and Fachbereich Physik, \\ Freie Universität Berlin, Arnimallee 14, 14195 Berlin, Germany \\ ${ }^{5}$ Center for Quantum Devices, Niels Bohr Institute, University of Copenhagen, DK-2100 Copenhagen, Denmark
}

(Dated: September 12, 2021)

\begin{abstract}
We explore higher-order topological superconductivity in an artificial Dirac material with intrinsic spin-orbit coupling. A mechanism for superconductivity due to repulsive interactions - pseudospin pairing - has recently been shown to result in higher-order topology in Dirac systems past a minimum chemical potential [1]. Here we apply this theory through microscopic modelling of a superlattice potential imposed on an inversion symmetric hole-doped semiconductor heterostructure, and extend previous work to include the effects of spin-orbit coupling. We find spin-orbit coupling enhances interaction effects, providing an experimental handle to increase the efficiency of the superconducting mechanism. We find that the phase diagram, as a function of chemical potential and interaction strength, contains three superconducting states - a first-order topological $p+i p$ state, a second-order topological spatially modulated $p+i \tau p$ state, and a second-order topological extended $s$-wave state, $s_{\tau}$. We calculate the symmetry-based indicators for the $p+i \tau p$ and $s_{\tau}$ states, which prove these states possess second-order topology. Exact diagonalisation results are presented which illustrate the interplay between the boundary physics and spin orbit interaction. We argue that this class of systems offer an experimental platform to engineer and explore first and higher-order topological superconducting states.
\end{abstract}

I. Introduction

II. Single particle effective Hamiltonian
A. Spin-orbit coupled honeycomb superlattice

B. Effective Dirac Hamiltonian

\section{Coulomb Matrix Elements}

IV. Screening

V. Superconducting instabilities
A. Interactions in the Cooper channel
B. Gap equation
C. Explicit solution and phase diagram

\footnotetext{
*h.scammell@unsw.edu.au
}

22 VI. Competing Instabilities

2 2з VII. Topological properties of the superconducting phases

$24 \quad$ A. Symmetry-based indicators for $p+i \tau p$ and $s_{\tau}$ phases 12

3

3

$4 \quad 26$ VIII. Discussion 
42 Higher-order topological superconductors are superconducting 43 phases which exhibit gapless corner (hinge) modes in two (three) ${ }_{44}$ dimensions protected by spatial symmetries and the bulk gap, and 45 have recently attracted immense interest [2-29]. It was recently pro${ }_{46}$ posed that Dirac materials, with purely repulsive interactions and ${ }_{47}$ sufficiently localised orbitals, intrinsically give rise to higher-order ${ }_{48}$ topological superconductivity [1]. We will refer to this as mecha49 nism as pseudospin pairing.

${ }_{50}$ Superlattices are a promising platform for this mechanism [30], ${ }_{51}$ since they allow the experimental study of materials with tunable ${ }_{52}$ lattice constants, atomic orbitals and interactions [31], and have been ${ }_{53}$ extensively explored in the context of optical lattices [32-35] and van ${ }_{54}$ der Waals heterostructures [36-42]. Recently, significant experimen${ }_{55}$ tal progress has also been made in forming honeycomb superlattices ${ }_{56}$ in patterned semiconductor heterostructures [43-48]. Motivated by ${ }_{57}$ these developments, in this paper we discuss a $p$-type quantum well ${ }_{58}$ overlaid with a periodic potential with honeycomb symmetry (see ${ }_{59}$ e.g. Refs [49-53]) as an explicit realisation of the pseudospin pairing 60 mechanism. Here we extend the theory to include the influence of ${ }_{61}$ intrinsic spin-orbit coupling. The superlattice potential gives rise to ${ }_{62}$ Dirac band crossings at the $K, K^{\prime}$ points; accounting for the intrinsic ${ }_{63}$ spin-orbit coupling gives rise to a spin-dependent mass for the Dirac ${ }_{64}$ fermions, opening up a $\mathbb{Z}_{2}$ topological bandgap. The low energy 65 effective theory is equivalent to the Kane-Mele model for a topolog${ }_{66}$ ical insulator [52-54], with an effective Dirac velocity controlled by ${ }_{67}$ the strength of spin-orbit coupling. We find that spin-orbit coupling ${ }_{68}$ enhances the superconducting instability and provides an additional ${ }_{69}$ handle to manipulate the topological superconducting phases.

70 We present results specifically for a model of an artificial honey71 comb lattice based on a nanopatterned hole-doped semiconductor ${ }_{72}$ quantum well, having in mind the fact that in this situation there ${ }_{11}$ 73 is a high degree of experimental control over the electron-electron 1 ${ }_{74}$ interaction as well as the band structure. However, our field theory
2675 treatment is generic and we anticipate our the results are relevant to 76 a number of other Dirac materials, in which similar spin-orbit physics 77 is present alongside localised orbitals. Unconventional superconduc78 tivity has recently been observed in twisted transition metal dichalo79 genides (TMDs) [55], which are Dirac systems where spin-orbit cou80 pling plays an important role. Theoretical studies of twisted TMDs, ${ }_{81}$ e.g. Ref. [56], have suggested effective models for the superlattice 82 potential similar to the one we examine in the present paper. Su${ }_{83}$ perconductivity has also been seen in the intrinsic heterostructure ${ }_{84} \mathrm{Ba}_{6} \mathrm{Nb}_{11} \mathrm{~S}_{28}$, a material which can be modelled as a stack of decou${ }_{85}$ pled $\mathrm{NbS}_{2}$ layers subjected to a superlattice potential arising from ${ }_{86}$ the $\mathrm{Ba}_{3} \mathrm{NbS}_{5}$ spacer layers [57]. Other than superlattice systems, ${ }_{87}$ superconductivity is seen in spin-orbit coupled topological materials ${ }_{88}$ including $\mathrm{Pb}_{1 / 3} \mathrm{TaS}_{2}$ [58], few-layer stanene [59], monolayer TMDs 89 [60-64], doped topological insulators [65-73], and recently discovered 90 vanadium-based kagome metals [74-93].

${ }_{91}$ We determine the phase diagram of the system as a function of ${ }_{92}$ chemical potential and interaction strength. Employing physically ${ }_{93}$ realistic parameters, we find three adjacent superconducting phases 94 - one first-order topological $p+i p$ intervalley, and two higher-order 95 topological: $s_{\tau}$ intervalley, and $p+i \tau p$ intravalley - with spin-orbit ${ }_{96}$ coupling entangling the valley and spin polarisation of the Cooper ${ }_{97}$ pairs. The $s_{\tau}$ state is similar to the $s_{ \pm}$state discussed in the context 98 of iron-based superconductors, which consists of $s$-wave pairing but 99 with a gap that has opposite signs at the hole and electron pockets 100 [94-99]; here, the valley structure imposes that $s$-wave state changes 101 sign under exchange of the valleys.

The $p+i \tau p$ and $s_{\tau}$ pairing instabilities satisfy a simple criterion for ${ }_{03}$ second-order topology derived from symmetry-based indicators [5104 7]: by counting the inversion eigenvalues of the valence and conduc105 tion bands in the normal state, we prove that if a superconducting 106 instability with odd inversion parity opens a full excitation gap in a 107 hole-doped Kane-Mele honeycomb system, then the resulting super108 conducting state must have second-order topology, hosting Kramers 109 pairs of Majorana corner modes. This conclusion holds for weak 10 spin-orbit coupling much smaller than the bandwidth, and for the 11 onset of superconductivity where the superconducting order param112 eter is the smallest energy scale. The second-order topological phase ${ }_{113}$ persists as long as increasing the superconducting order parameter 114 does not close the bulk excitation gap.

In Section II, we will outline how the effective Dirac theory arises 16 from the superlattice imposed on the 2D hole gas. In Section III, we 117 will discuss the form of the symmetry-allowed interactions for the 

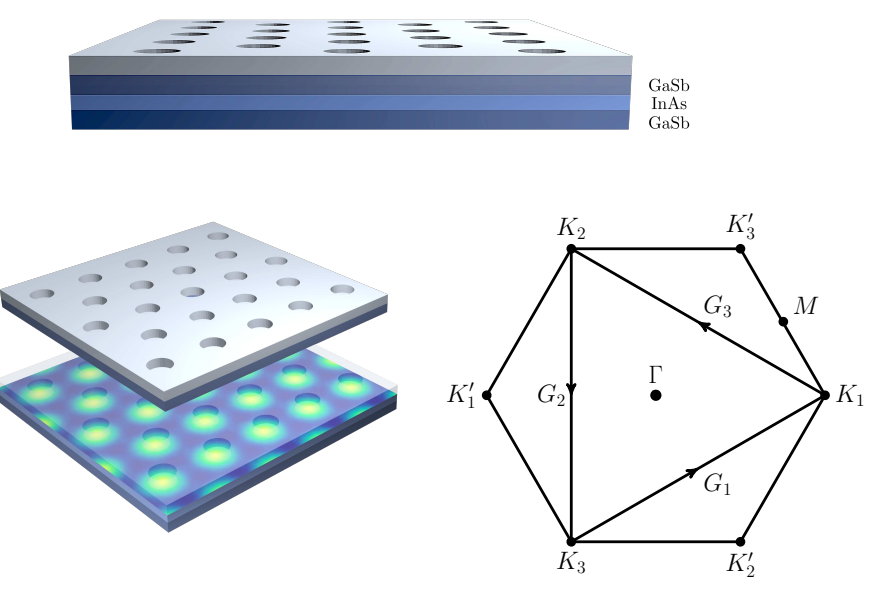

FIG. 1: Schematic view of the honeycomb superlattice patterned on the heterostructure $2 \mathrm{DHG}$ - a patterned dielectric or gate is placed on a quantum well e.g. GaSb-InAs-GaSb. Superlattice Brillouin zone: the reciprocal lattice vectors $\boldsymbol{G}_{i}$ connect zone corners corresponding to $\boldsymbol{K}_{j}$, and connect corners corresponding to $\boldsymbol{K}_{j}^{\prime}$, the parity reflections of $\boldsymbol{K}_{j}$.
${ }_{140}$ We consider a $p$-type quantum well, having in mind for e.g. a ${ }_{141}$ GaSb-InAs-GaSb heterojunction (see Fig. 1). The hole gas expe142 riences a potential well, arising from the band-bending along the ${ }_{143}$ growth direction of the heterojunction, confining the holes along the ${ }_{144} z$-direction leaving a two dimensional hole gas (2DHG) unconfined in 145 the $x y$ plane. The hole states are formed from $p_{\frac{3}{2}}$ orbitals and can be 146 described by the Luttinger Hamiltonian involving spin- $\frac{3}{2}$ operators ${ }_{147} \boldsymbol{S}$ in the axial approximation, i.e. $U(1)$ symmetry in-plane [100]. 148 Ignoring the cubic anisotropy of the zincblende lattice, which has a 149 weak effect for the carrier densities we consider, the Hamiltonian is

$$
H_{2 D H G}=\frac{1}{2 m_{e}}\left[\left(\gamma_{1}+\frac{5}{2} \gamma_{2}\right) \boldsymbol{p}^{2}-2 \gamma_{2}(\boldsymbol{p} \cdot \boldsymbol{S})^{2}\right]+W_{c}(z)
$$

${ }_{150}$ The $\gamma_{i}$ are the Luttinger parameters; in what follows we shall use ${ }_{151}$ parameters for InAs, presented in Table I. In this work we model the 152 confinement as a rectangular infinite well of width $d$,

$$
W_{c}(z)= \begin{cases}0, & z \in(-d / 2, d / 2) \\ \infty, & \text { otherwise }\end{cases}
$$

118 effective Dirac system. Particularly important are the pseudospin- 153 The Hamiltonian (1) satisfies time-reversal and inversion symmetry, 119 dependent Hubbard interactions; we present numerical results for ${ }_{154}$ so each 2D subband is twofold degenerate. We consider densities for 120 these parameters based on explicit calculations. In Section IV, we 155 which only the lowest pair of subbands is occupied, and introduce 121 will analyse the screening properties of this system - screening plays 156 an effective spin- $\frac{1}{2}$ degree of freedom with Pauli matrices $s_{\mu}$.

122 a crucial role for superconducting pairing mechanism, as discussed ${ }_{157}$ Next, we consider the influence of a periodic electrostatic poten123 in the earlier work (for electrons, without spin-orbit coupling [30]). 158 tial, with honeycomb symmetry, on the 2DHG, i.e. the superlattice. 124 It was shown that the pseudospin-dependent Hubbard interactions ${ }_{159}$ Experimentally, this may be implemented by etching the pattern 125 are antiscreened (enhanced) by many-body effects; we analyse this 160 onto a metal plate or dielectric on top of the 2DHG. A minimal 126 phenomenon in the presence of spin-orbit coupling. In Section V, 161 model of the superlattice is given by [49],

127 we present the solution to the BCS gap equation using the screened 128 form of the interactions, and present a phase diagram of possible 129 superconducting states. In Section VII, we discuss the phenomenol${ }_{130}$ ogy of the possible superconducting phases, and present numerical 131 results describing the edge physics as well as symmetry indicators ${ }_{132}$ which confirm the higher topology of the $p+i \tau p$ and $s_{\tau}$ states.

\section{SINGLE PARTICLE EFFECTIVE HAMILTONIAN}

134 In this section, we will present the effective Dirac theory that arises 135 for a particular honeycomb superlattice system - $p$-type artificial 136 graphene - though aspects of the model apply generally. We briefly ${ }_{137}$ outline the schematics of artificial graphene, and in doing so establish ${ }_{138}$ the key parameters which may be tuned in experiment.

$$
W(\boldsymbol{r})=2 W_{0} \sum_{i} \cos \left(\boldsymbol{G}_{i} \cdot \boldsymbol{r}\right),
$$

162 where $\boldsymbol{G}_{1}=\boldsymbol{K}_{2}-\boldsymbol{K}_{1}, \boldsymbol{G}_{2}=\boldsymbol{K}_{3}-\boldsymbol{K}_{2}, \boldsymbol{G}_{3}=\boldsymbol{K}_{1}-\boldsymbol{K}_{3} ; \boldsymbol{K}_{1}=$ ${ }_{163} \frac{4 \pi}{3 L}(1,0), \boldsymbol{K}_{2}=\frac{4 \pi}{3 L} \frac{1}{2}(-1, \sqrt{3}), \boldsymbol{K}_{3}=\frac{4 \pi}{3 L} \frac{1}{2}(-1,-\sqrt{3})$, with (super)${ }_{164}$ lattice constant $L$, and magnitude of the electrostatic potential $W_{0}$.
TABLE I: Physical parameters for InAs.

\begin{tabular}{|ccc|}
\hline \hline Parameter & Details & Value \\
\hline$\gamma_{1}$ & Luttinger parameter & 20.4 \\
$\gamma_{2}$ & Luttinger parameter & 8.3 \\
$\gamma_{3}$ & Luttinger parameter & 9.1 \\
$m_{H}$ & Effective mass: $m_{e}\left(\gamma_{1}+\gamma_{2}\right)^{-1}$ & 0.0348 \\
$\epsilon_{r}$ & Dielectric constant & 14.6 \\
\hline
\end{tabular}


(a)

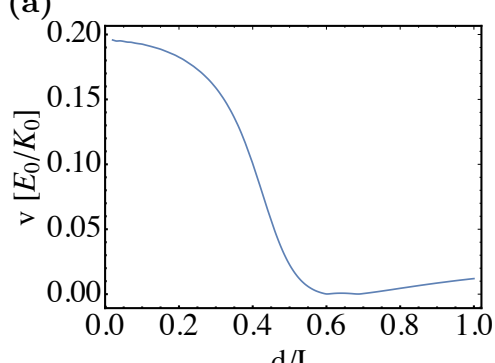

$\mathrm{d} / \mathrm{L}$ (b)

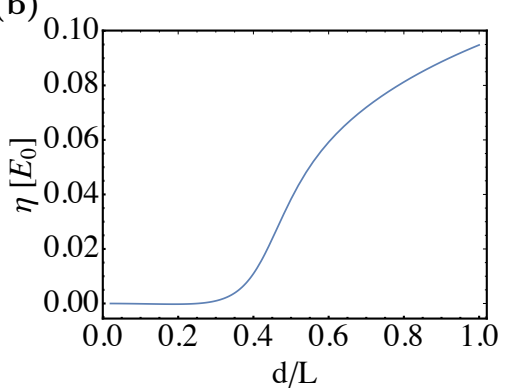

FIG. 2: Parameters of the effective Dirac Hamiltonian (4). (a) Dirac velocity $v$, in units of $E_{0} / K_{0}$. (b) spin-orbit gap $\eta$, in units of $E_{0}$.

165 The separation along the $z$-axis of the superlattice top gate from ${ }_{166}$ the $2 \mathrm{DHG}$ is $z_{0}$. Although $z_{0}$ plays a role [49], we will fix its value 167 and not consider it further. Moreover, we employ a minimal three ${ }_{168} K$-point grid for numerical diagonalisation of $H_{2 D H G}+W(\boldsymbol{r})$, which 169 is used to estimate the couplings entering the effective Dirac Hamil170 tonian (4). In this scheme, $W_{0}$ scales out, and so will not explicitly 171 appear as a free parameter in our analysis. The shortcomings of this 172 approximation are discussed further in Section V C in relation to the 173 phase diagram.

\section{B. Effective Dirac Hamiltonian}

175 Since the periodic potential $W(\boldsymbol{r})$ has the same symmetries as 176 the atomic potential in graphene, the bandstructure of the hole gas 177 with superlattice, i.e. $H_{2 D H G}+W(\boldsymbol{r})$, features Dirac cones at the 200 178 high symmetry points $\boldsymbol{K}_{i}$. Performing this diagonalisation explicitly 179 (see Appendix A), and expanding the resulting Hamiltonian about 180 the Dirac points, we arrive at the effective Dirac Hamiltonian with

${ }_{181}$ Kane-Mele mass term [54]

$$
H_{0}=\sum_{\boldsymbol{p}} \psi_{\boldsymbol{p}}^{\dagger}\left(v(\boldsymbol{\sigma} \cdot \boldsymbol{p}) \tau_{z}-\mu+\eta \sigma_{z} s_{z}\right) \psi_{\boldsymbol{p}}
$$

182

the Pauli matrices $\sigma_{i}, \tau_{i}$ and $s_{i}$ act on sub-lattice, valley, and the ${ }^{214}$ effects. 183 effective spin- $1 / 2$, and a chemical potential $\mu$ describes doping be- ${ }_{215}$ Holes have larger effective masses $m_{H}$ and moreover, the dis184 yond the Dirac points. For $\tau=1$, pseudospin up (down) corresponds ${ }_{216}$ persion is non-parabolic - becoming flatter due to an anticrossing 185 to sublattice $A(B)$, while at the opposite valley $\tau=-1$, pseudospin ${ }_{217}$ between heavy hole and light hole states (see Appendix A). Band 186 up (down) corresponds to sublattice $B(A)$. One may perform a uni- ${ }_{218}$ folding this anticrossing to the Dirac point generates flatter Dirac 187 tary transformation so that the pseudospin has the same definition ${ }_{219}$ bands [53], and correspondingly more localised orbitals; this is con188 at $\tau=-1$ as it does at $\tau=1$, but intermediate calculations are 220 trolled by ratio $d / L$. Such a handle is not available in the analogous 189 made more simple in the basis of (4). At the end of Section IV, we 221 electron-based superlattice honeycomb systems [30, 48].

TABLE II: Transformation properties of operators $\sigma_{i}, \tau_{i}$ and $s_{i}$ under the symmetries of the system: $2 \pi / 3$ and $\pi$ rotations $C_{3 z}$, $C_{2 z}, C_{2 x}$ (for completeness we include $C_{2 y}$ ), and time reversal $\mathcal{T}$.

\begin{tabular}{|c|ccccc|}
\hline \hline & $C_{3 z}$ & $C_{2 z}$ & $C_{2 x}$ & $C_{2 y}$ & $\mathcal{T}$ \\
\hline$s_{z}$ & $s_{z}$ & $s_{z}$ & $-s_{z}$ & $-s_{z}$ & $-s_{z}$ \\
$\sigma_{z}$ & $\sigma_{z}$ & $\sigma_{z}$ & $-\sigma_{z}$ & $-\sigma_{z}$ & $-\sigma_{z}$ \\
$\tau_{z}$ & $\tau_{z}$ & $-\tau_{z}$ & $\tau_{z}$ & $-\tau_{z}$ & $-\tau_{z}$ \\
$\tau_{ \pm}$ & $\tau_{ \pm}$ & $\tau_{\mp}$ & $\tau_{ \pm}$ & $\tau_{\mp}$ & $\tau_{\mp}$ \\
$\sigma_{ \pm}$ & $e^{2 i \theta_{ \pm}} \sigma_{ \pm}$ & $\sigma_{ \pm}$ & $\sigma_{\mp}$ & $\sigma_{\mp}$ & $\sigma_{\mp}$ \\
\hline
\end{tabular}

190 shall change to the alternative basis as it makes aspects of our final 191 results clearer.

192 The symmetries of the system are $2 \pi / 3$ and $\pi$ rotations, and time 193 reversal. The resulting transformation properties of the operators ${ }_{94} \sigma_{i}, \tau_{i}$ and $s_{i}$ are given in Table II.

195 The time-reversal invariant mass term $\eta \sigma_{z} s_{z}$ arises from the spin196 orbit interaction and is absent in $n$-type artificial lattices. This term 197 gives rise to a $\mathbb{Z}_{2}$ topological insulating state. In the Appendix we 198 show that, in an effective tight-binding description of the artificial 199 lattice, this term arises due to a spin-dependent complex next near200 est neighbor hopping which is equivalent to two copies of the Hal201 dane model. While the effective Dirac theory is identical to that of 202 the Kane-Mele model, the hopping phases in the real space descrip203 tion are different, due to the fact that the mass term arises from a 204 spin-orbit interaction quadratic in momentum, rather than a linear ${ }_{20}$ Rashba spin-orbit interaction.

Performing exact diagonalisation of the Luttinger Hamiltonian 208 Dirac Hamiltonian (4). We plot the Dirac velocity $v$ and spin-orbit 209 mass gap $\eta$ as a function of $d / L$ in Fig. 2, in terms of the scale ${ }_{210} E_{0}=K_{0}^{2} /\left(2 m_{H}\right)$, with $K_{0}=\left|\boldsymbol{K}_{i}\right|$. There we see that the effective ${ }_{211}$ Dirac velocity $v$ and the spin-orbit mass gap $\eta$ depend strongly on (4) 212 the ratio $d / L$. The Dirac velocity can be significantly reduced by in${ }_{213}$ creasing $d / L$, flattening the Dirac bands and enhancing interaction

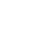


228 ing earlier results on these models by including spin $[1,30]$. The 229 form of these Hubbard interactions are constrained by the symmetry 230 transformations of Table II. Here we directly compute numerically

224 tion in the effective Dirac theory. By writing the Coulomb interac- ${ }_{232}$ Using the wavefunctions, $|\boldsymbol{k}, s, \sigma, \tau\rangle$, obtained from diagonalisation 225 tion in the basis of states near the $K$ and $K^{\prime}$ points, we find that ${ }_{23}$ of the InAs 2DHG subject to superlattice potential $W(\boldsymbol{r})$ (3), i.e. 226 the Coulomb repulsion contains a short range Hubbard part, which ${ }_{234} H_{2 D H G}+W(\boldsymbol{r})$, and expanding near the Dirac points, we explicitly ${ }_{227}$ depends on the pseudospin $\sigma$, valley $\tau$ and effective spin $s$, extend- ${ }_{235}$ compute the matrix elements of the Coulomb interaction,

$$
\begin{aligned}
\hat{V} & =\left\langle\boldsymbol{k}_{1}, s_{4}, \sigma_{4}, \tau_{4}\left|\otimes\left\langle\boldsymbol{k}_{3}, s_{3}, \sigma_{3}, \tau_{3}\left|\frac{e^{2}}{2 \varepsilon_{r}\left|\boldsymbol{r}-\boldsymbol{r}^{\prime}\right|}\right| \boldsymbol{k}_{2}, s_{2}, \sigma_{2}, \tau_{2}\right\rangle \otimes\right| \boldsymbol{k}_{1}, s_{1}, \sigma_{1}, \tau_{1}\right\rangle \equiv \frac{2 \pi e^{2}}{\epsilon_{r} q}+\hat{V}_{I}+\hat{V}_{I I}, \\
\hat{V}_{I} & =\left(v_{00} \sigma_{0} \otimes \sigma_{0}+v_{44} \tau_{z} s_{z} \otimes \tau_{z} s_{z}\right)+\left(v_{33} \tau_{z} \otimes \tau_{z}+v_{77} s_{z} \otimes s_{z}\right) \sigma_{z} \otimes \sigma_{z}+\left(v_{12}+v_{56} \tau_{z} s_{z} \otimes \tau_{z} s_{z}\right)\left(\sigma_{+} \otimes \sigma_{-}+\sigma_{-} \otimes \sigma_{+}\right) \\
& +v_{07}\left(\sigma_{0} \otimes \sigma_{z} s_{z}+\sigma_{z} s_{z} \otimes \sigma_{0}\right)+v_{47}\left(\tau_{z} s_{z} \otimes \sigma_{z} s_{z}+\sigma_{z} s_{z} \otimes \tau_{z} s_{z}\right), \\
\hat{V}_{I I} & =\left[u_{00} \sigma_{0} \otimes \sigma_{0}+u_{33} s_{z} \sigma_{z} \otimes s_{z} \sigma_{z}+u_{12}\left(\sigma_{+} \otimes \sigma_{-}+\sigma_{-} \otimes \sigma_{+}\right)+u_{03}\left(\sigma_{0} \otimes \sigma_{z} s_{z}+\sigma_{z} s_{z} \otimes \sigma_{0}\right)\right]\left(\tau_{+} \otimes \tau_{-}+\tau_{-} \otimes \tau_{+}\right),
\end{aligned}
$$

${ }_{236}$ Here $\boldsymbol{q}=\boldsymbol{k}_{1}-\boldsymbol{k}_{3}$, and subscripts $I$ and $I I$ denote in- 253 where $G\left(q_{0}, \boldsymbol{q}\right)$ is the single particle Green's function. In general, the ${ }_{237}$ travalley ( $\tau$-diagonal) and intervalley ( $\tau$-off-diagonal) interactions. ${ }_{254}$ vertices $J^{\mu}, J^{\nu}$ can be any matrix $\sigma^{i} \tau^{j} s^{k}$ which appears in the bare ${ }_{238}$ The vertices appearing in the bare interactions are $J_{I}^{\mu} \in{ }_{255}$ interactions of the form $V_{\mu \nu} J^{\mu} \otimes J^{\nu}$. In this paper we will restrict our $239\left\{\mathbb{1}, \sigma_{ \pm}, \tau_{z} \sigma_{z}, \tau_{z} s_{z}, \tau_{z} s_{z} \sigma_{ \pm}, \sigma_{z} s_{z}\right\}, J_{I I}^{\mu} \in\left\{\mathbb{1}, \sigma_{ \pm}, \sigma_{z} s_{z}\right\} \otimes \tau_{ \pm}$, which 256 attention to the case of static screening, so we neglect the frequency 240 defines the adjoint basis. Using these vertices, the interactions are 257 dependence of the polarisation operator and set $p_{0}=0$.

${ }^{241}$ parametrized $\hat{V}_{I}^{0}=v_{\mu \nu} J_{I}^{\mu} \otimes J_{I}^{\nu}$ and $\hat{V}_{I I}^{0}=u_{\mu \nu} J_{I I}^{\mu} \otimes J_{I I}^{\nu}$, which ${ }_{258}$ As shown in Section III, the vertices appearing in the bare in${ }_{242}$ defines the notation in Eq. (5). In Figure 3 we plot the dependence 259 teractions (5) are $J_{I}^{\mu} \in\left\{\mathbb{1}, \sigma_{ \pm}, \tau_{z} \sigma_{z}, \tau_{z} s_{z}, \tau_{z} s_{z} \sigma_{ \pm}, \sigma_{z} s_{z}\right\}, J_{I I}^{\mu} \in$ 243 of the coefficients $\left\{v_{\mu \nu}, u_{\mu \nu}\right\}$ on the spin-orbit parameter, $d / L$. $260\left\{\mathbb{1}, \sigma_{ \pm}, \sigma_{z} s_{z}\right\} \otimes \tau_{ \pm}$, which defines the adjoint basis. In this basis, ${ }_{261}$ the tensor form of the static polarisation operator becomes,

\section{SCREENING}

245

In this section we discuss how the bare Coulomb interactions (5) 246 are modified by screening. A standard approach for analysing the 247 feedback of many body effects on interactions is the Random Phase 248 Approximation [1, 30, 101-104], which involves resumming the in249 finite series of bubble diagrams which contribute corrections to the 250 bare Coulomb interaction.

where $\Pi^{\alpha \gamma}$ is the particle-hole polarisation operator, given by

$$
\begin{gathered}
i \Pi^{\alpha \gamma}\left(p_{0}, \boldsymbol{p}\right)=\operatorname{Tr} \int J^{\alpha} G\left(q_{0}+p_{0}, \boldsymbol{q}+\boldsymbol{p}\right) J^{\gamma} G\left(q_{0}, \boldsymbol{q}\right) \frac{d q_{0} d^{2} \boldsymbol{q}}{(2 \pi)^{3}}, \\
G\left(q_{0}, \boldsymbol{q}\right)=\frac{1}{q_{0}+\mu-v \tau_{z} \boldsymbol{q} \cdot \boldsymbol{\sigma}-\eta \sigma^{z} s^{z}+i 0 \operatorname{sgn}\left(q_{0}\right)}
\end{gathered}
$$

$$
\begin{aligned}
\hat{\Pi}_{I}\left(p_{0}=0, \boldsymbol{p}\right) & =\Pi^{\mu \nu}(0, \boldsymbol{p}) J_{I}^{\mu} J_{I}^{\nu}, \\
\hat{\Pi}_{I I}\left(p_{0}=0, \boldsymbol{p}\right) & =\Pi^{\mu \nu}(0, \boldsymbol{p}) J_{I I}^{\mu} J_{I I}^{\nu} .
\end{aligned}
$$

${ }_{262}$ The quantities $\Pi^{\mu \nu}(0, \boldsymbol{p})$ are evaluated in the Appendix B. We 263 find that only three independent polarisation operators emerge. To 264 gain some insight into their physical meaning, we shall discuss 265 their behavior in the long wavelength limit, $q \rightarrow 0$. First, we 266 find a term $\Pi_{0} \rightarrow-\mu N /(2 \pi)$, this term corresponds to the usual 267 density-density (Thomas-Fermi) screening, i.e. the vertices cou(6) ${ }^{268}$ pling to a negative polarisation operator are weakened by screen269 ing. Second, $\Pi_{z} \rightarrow \mu N /(2 \pi)$, which corresponds to a pseudospin 270 dipole-dipole antiscreening, first discussed in [30] - the positive sign 271 here causes an enhancement of the couplings $v_{33}\left(\tau_{z} \sigma_{z} \otimes \tau_{z} \sigma_{z}\right)$ and ${ }_{272} v_{77}\left(s_{z} \sigma_{z} \otimes s_{z} \sigma_{z}\right)$, i.e. those proportional to $\sigma_{z} \otimes \sigma_{z}$, which as we shall 273 later see promotes an intravalley $p+i \tau p$ higher topological super${ }_{274}$ conductivity. Similarly, an antiscreening occurs for intervalley terms 
(a)

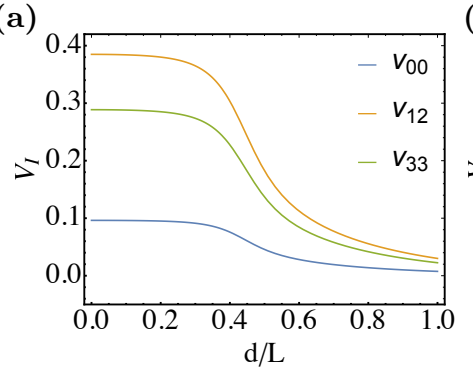

(b)

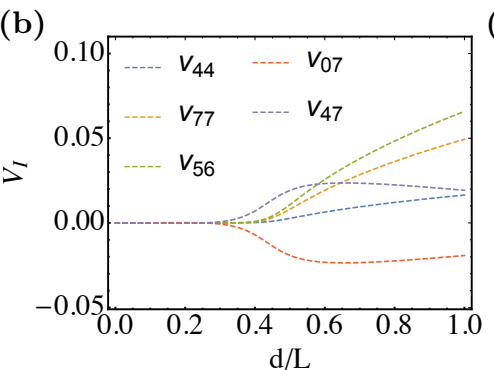

(c)

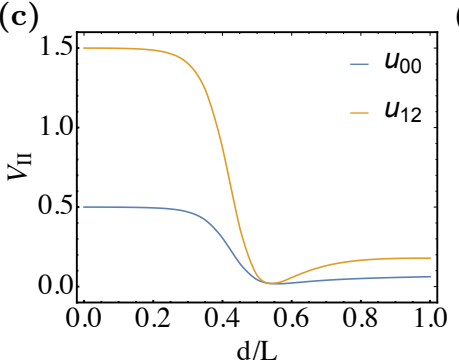

(d)

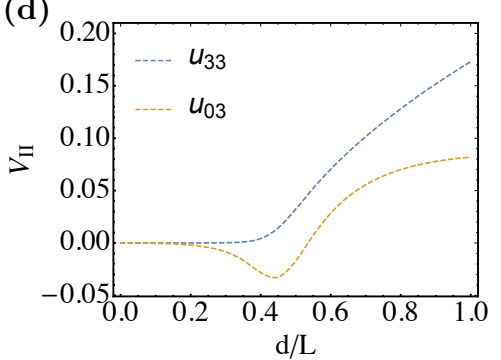

FIG. 3: (a) Spin-independent matrix elements of $V_{I}$. Solid lines: blue, orange, green $=v_{00}, v_{33}, v_{12}$. (b) Spin-dependent matrix elements of $V_{I}$. Dashed lines: blue, orange, green, red and purple $=v_{44}, v_{77}, v_{56}, v_{07}, v_{47}$. (c) Spin-independent matrix elements of $V_{I I}$. Solid lines: Blue, green $=u_{00}, u_{12}$. (d) Spin-dependent matrix elements of $V_{I I}$. Dashed lines: orange, red $=u_{33}, u_{03}$. In units of $2 \pi e^{2} /\left(\epsilon_{r} K_{0}\right)$

${ }_{275} u_{00} \sigma_{0} \tau_{ \pm} \otimes \sigma_{0} \tau_{\mp}, u_{33} s_{z} \tau_{ \pm} \otimes s_{z} \tau_{\mp}$, which acts to favour the higher topo- ${ }_{296}$ 276 logical intervalley $s_{\tau}$ state. We shall elaborate on this phenomenon 277 in the following subsection. Third, we have $\Pi_{\eta}=\rightarrow \eta N /(2 \pi)$, a di278 rect result of the spin-orbit coupling. Last, we have $\Pi_{ \pm} \propto e^{i \theta_{p}}$, this 279 Hall-like response, with momentum dependence, promotes interac280 tion matrix elements that were otherwise not present in the bare 281 interaction structure (5).

Inverting the matrix equation (6) is now straightforward and gives,
The interactions transform as

$$
\begin{aligned}
\hat{\tilde{V}}_{I}^{R} & =v_{\mu \nu}^{R}\left(P J_{I}^{\mu} P^{\dagger}\right)\left(P J_{I}^{\nu} P^{\dagger}\right) \\
\hat{\tilde{V}}_{I I}^{R} & =u_{\mu \nu}^{R}\left(P J_{I I}^{\mu} P^{\dagger}\right)\left(P J_{I I}^{\nu} P^{\dagger}\right) \\
P J_{I}^{\mu} P^{\dagger} & \in P\left\{\mathbb{1}, \tau_{z} s_{z}, \tau_{z} \sigma_{z}, \sigma_{z} s_{z}, \sigma_{ \pm}, \tau_{z} s_{z} \sigma_{ \pm}\right\} P^{\dagger} \\
& =\left\{\mathbb{1}, \tau_{z} s_{z}, \sigma_{z}, \tau_{z} \sigma_{z} s_{z}, \sigma_{ \pm}^{\tau}, \tau_{z} s_{z} \sigma_{ \pm}^{\tau}\right\} \\
P J_{I I}^{\mu} P^{\dagger} & \in P\left\{\mathbb{1}, \sigma_{z} s_{z}, \sigma_{+}, \sigma_{-}\right\} \otimes \tau_{ \pm} P^{\dagger} \\
& =\left\{\sigma_{x}, \pm i \sigma_{y} s_{z},\left(\sigma_{0} \pm \sigma_{z}\right) / 2,\left(\sigma_{0} \mp \sigma_{z}\right) / 2\right\} \otimes \tau_{ \pm} .
\end{aligned}
$$

${ }_{297}$ Here $\sigma_{ \pm}^{\tau} \equiv \sigma_{x} \pm i \tau_{z} \sigma_{y}$, and in $P J_{I I}^{\mu} P^{\dagger}$, the \pm indices in pseudospin 298 and valley are connected.

${ }_{283}$ with superscript $R$ to denote the RPA renormalised values. De${ }_{284}$ spite there being a closed form analytic expression, we do not pro285 vide the full expressions for matrix elements $v_{\mu \nu}, u_{\mu \nu}$ since they are 286 lengthy and unenlightening. The expression (10) defines the RPA${ }_{287}$ renormalised interaction structure which we use to search for super288 conducting and magnetic instabilities.

${ }_{289}$ Up until this point we have worked in a particular basis for the 290 single particle Hamiltonian (4), which allowed for straightforward 291 evaluation of the polarisation operators.However, from this point on 292 we work in a more physical basis, which will make our later discussion 293 of the superconducting gap structure more transparent. Performing 294 a unitary transformation, with $P=\frac{1}{2}\left(\tau_{0}+\tau_{z}\right)+\frac{1}{2}\left(\tau_{0}-\tau_{z}\right) \sigma_{x}$, we 295 obtain

$$
\widetilde{H}_{0}=P H_{0} P^{\dagger}=v p_{x} \sigma_{x} \tau_{z}+v p_{y} \sigma_{y}+\eta s_{z} \sigma_{z} \tau_{z} .
$$

299

\section{SUPERCONDUCTING INSTABILITIES}

300 In this section we analyse superconductivity resulting from the 301 renormalised pseudospin dependent couplings. At a finite doping 302 away from the Dirac point, the states at the Fermi surface are not ${ }_{303}$ pseudospin eigenstates, but band eigenstates. The interactions (10) 304 are therefore be rewritten in the basis of band indices, and further305 more since we are only interested in Fermi surface instabilities, we 306 project onto the upper band (i.e. only include states at the Fermi 307 surface). The BCS gap equation is then used to calculate $T_{c}$ for ${ }_{308}$ pairing between these states.

\section{A. Interactions in the Cooper channel}

310 To find the superconducting instability, we are interested only in 311 states near the Fermi surface, which participate in pairing. Hence, 312 we keep only states in the upper band of (9), the eigenstates of which 
313 are given by

$$
|\boldsymbol{k}, \tau\rangle=\frac{1}{\sqrt{2}} e^{i \boldsymbol{k} \cdot \boldsymbol{r}}\left(w_{\tau, s}^{a}(k)|a\rangle+w_{\tau, s}^{b}(k) e^{i \tau \theta_{\boldsymbol{k}}}|b\rangle\right),
$$

314 where $|a\rangle,|b\rangle$ are the $\sigma^{z}$ eigenstates, which are localised on the ${ }_{315} A$ and $B$ sites respectively, and the wavefunction components, ${ }_{316} w_{\tau, s}^{a}(k)=v k / \sqrt{2 \epsilon_{k}\left(\epsilon_{k}-s \tau \eta\right)}, w_{\tau, s}^{b}(k)=\left(\epsilon_{k}-s \tau \eta\right) w_{\tau, s}^{a}(k) /(\tau v k)$.

317 To obtain the interactions between Cooper pairs, we perform the 318 following process: $(i)$ project the RPA interaction tensor (10) onto 319 the upper band using (11), (ii) impose the scattering conditions of 320 the Cooper channel $\boldsymbol{k}_{1}=-\boldsymbol{k}_{3}, \boldsymbol{k}_{2}=-\boldsymbol{k}_{4}$, i.e. $\theta_{k_{3}}=\pi+\theta_{k_{1}}, \theta_{k_{4}}=$ ${ }_{321} \pi+\theta_{k_{2}}$, (iii) restrict all momenta to lie on the Fermi surface $\left|\boldsymbol{k}_{i}\right|=$ $322 k_{F}$. The interactions then only have angular dependence, and we ${ }_{323}$ decompose the resulting Cooper interaction into partial waves with 324 different angular momentum. The result is the coupling between 325 Cooper pairs with a given angular momentum.

326 Performing such a procedure, we arrive at the couplings in angular 327 momentum channels $\ell=0, \pm 1$ (higher-order channels are negligible 328 or zero),

$$
\begin{aligned}
\hat{\mathcal{V}}_{\ell=0} & =\tilde{g}_{0}+\tilde{g}_{1} \tau_{z} \otimes \tau_{z}+\tilde{g}_{2} s_{z} \otimes s_{z}+\tilde{g}_{3} s_{z} \tau_{z} \otimes s_{z} \tau_{z} \\
& +\left(\tilde{j}_{0}+\tilde{j}_{1} s_{z} \otimes s_{z}\right)\left(\tau_{x} \otimes \tau_{x}+\tau_{y} \otimes \tau_{y}\right) \\
\hat{\mathcal{V}}_{\ell= \pm 1} & =g_{0}+g_{1} \tau_{z} \otimes \tau_{z}+g_{2} s_{z} \otimes s_{z}+g_{3} s_{z} \tau_{z} \otimes s_{z} \tau_{z} \\
& +\ell\left(g_{4}+g_{5} s_{z} s_{z}\right)\left(\tau_{0} \tau_{z}+\tau_{z} \tau_{0}\right) \\
& +\left(j_{0}+j_{1} s_{z} \otimes s_{z}+\ell j_{2}\left(s_{0} \otimes s_{z}+s_{z} \otimes s_{0}\right)\right)\left(\tau_{x} \otimes \tau_{x}+\tau_{y} \otimes \tau_{y}\right)
\end{aligned}
$$

${ }_{329}$ The coefficients $g_{i}, j_{i}, \tilde{g}_{i}, \tilde{j}_{i}$ are functions of chemical potential $\mu$ 330 due to the screening effects, as well as the well width to lattice spac331 ing ratio $d / L$, which controls the strength of the spin-orbit depen332 dent couplings. The (un)tilded couplings correspond to the $(\ell= \pm 1)$ ${ }_{333} \ell=0$ partial wave channels. They also depend on the microscopic ${ }_{334}$ parameters of the 2DHG; we have evaluated these quantities numer335 ically for an InAs 2DHG. The matrix elements $g_{i}$ denote intravalley 336 scattering processes, while $j_{i}$ represent intervalley scattering.

\section{B. Gap equation}

The mean field Hamiltonian, which accounts for all pairing possi339 bilities, is

$$
\begin{aligned}
& \mathcal{H}_{M F}=\sum_{\boldsymbol{k}, s, \tau} \varepsilon_{\boldsymbol{k}} \widetilde{\psi}_{\boldsymbol{k} s \tau}^{\dagger} \widetilde{\psi}_{\boldsymbol{k} s \tau}+\frac{1}{2} \sum_{\boldsymbol{k}, s, \tau, s^{\prime}, \tau^{\prime}} \widetilde{\psi}_{\boldsymbol{k} s \tau}^{\dagger}\left(\Delta_{\boldsymbol{k}}\right)_{s \tau, s^{\prime} \tau^{\prime}} \widetilde{\psi}_{-\boldsymbol{k} s^{\prime} \tau^{\prime}}^{\dagger}+\text { h.c. } \\
& +\frac{1}{2}\left(\Delta_{\boldsymbol{k}}^{\dagger}\right)_{s_{1} \tau_{1}, s_{3} \tau_{3}}\left(\mathcal{V}^{-1}\right)_{\boldsymbol{k}, \boldsymbol{p} ; s_{1} \tau_{1} s_{2} \tau_{2} s_{3} \tau_{3} s_{4} \tau_{4}}\left(\Delta_{\boldsymbol{p}}\right)_{s_{4} \tau_{4}, s_{2} \tau_{2}}
\end{aligned}
$$

${ }_{340}$ where $\widetilde{\psi}_{\boldsymbol{k} s \tau}^{\dagger}$ is the hole creation operator for the upper band. We ${ }_{341}$ parametrize the gap in the standard form, collecting the spin, valley 342 and angular momentum structure into a tensor $d_{\ell}^{\mu \nu}$,

$$
\Delta_{k}=\sum_{\mu \nu, l} d_{\ell}^{\mu \nu} s_{\mu} \tau_{\nu} e^{-i \ell \theta_{k}}\left(\tau_{y} s_{y}\right)
$$

${ }_{343}$ The spin and valley structure follows from the usual singlet-triplet 344 decomposition (dropping the angular momentum index $\ell$ ) [105],

$$
\begin{aligned}
d^{\mu \nu} & =d_{s}^{\mu} \otimes d_{v}^{\nu}, & & \\
d_{s}^{x} & =\frac{1}{2}(|\uparrow \uparrow\rangle-|\downarrow \downarrow\rangle) & d_{s}^{y} & =\frac{1}{2 i}(|\uparrow \uparrow\rangle+|\downarrow \downarrow\rangle) \\
d_{s}^{z} & =-\frac{1}{2}(|\uparrow \downarrow\rangle+|\downarrow \uparrow\rangle) & d_{s}^{0} & =\frac{1}{2}(|\uparrow \downarrow\rangle-|\downarrow \uparrow\rangle), \\
d_{v}^{x} & =\frac{1}{2}(|++\rangle-|--\rangle) & d_{v}^{y} & =\frac{1}{2 i}(|++\rangle+|--\rangle) \\
d_{v}^{z} & =-\frac{1}{2}(|+-\rangle+|-+\rangle) & d_{v}^{0} & =\frac{1}{2}(|+-\rangle-|-+\rangle) .
\end{aligned}
$$

where subscript $s$ indicates spin and $v$ indicates valley. The BCS 346 gap equation is given by

$$
\begin{aligned}
d_{\ell}^{\mu \nu} & =-G_{\mu \nu ; \delta \gamma}^{\ell} d_{\ell}^{\delta \gamma} \int_{0}^{\varepsilon_{c}} \frac{N d \varepsilon}{2 \pi v^{2}} \frac{\varepsilon}{2 E\left(d_{\ell}^{\delta \gamma}\right)} \tanh \left(\frac{E\left(d_{\ell}^{\delta \gamma}\right)}{2 T}\right), \\
E\left(d_{\ell}^{\delta \gamma}\right) & =\sqrt{(\varepsilon-\mu)^{2}+\left|d_{\ell}^{\delta \gamma}\right|^{2}}
\end{aligned}
$$

347 where the matrix $G_{\mu \nu ; \delta \gamma}$ is given by

$$
G_{\mu \nu ; \delta \gamma}^{\ell} \equiv \frac{1}{4}\left(\hat{s}_{\mu} \hat{s}_{y}\right)_{a c}^{\dagger}\left(\hat{\tau}_{\nu} \hat{\tau}_{y}\right)_{a^{\prime} c^{\prime}}^{\dagger}\left(\hat{\mathcal{V}}_{\ell}\right)_{a b c d ; a^{\prime} b^{\prime} c^{\prime} d^{\prime}}\left(\hat{s}_{\delta} \hat{s}_{y}\right)_{b d}\left(\hat{\tau}_{\gamma} \hat{\tau}_{y}\right)_{b^{\prime} d^{\prime}}
$$

348 To determine the dominant instability $d^{\mu \nu}$, we find the gap func349 tion with highest $T_{c}$ via the eigenvalue problem (with eigenvalue $\left.350 \lambda_{\mu \nu}^{\ell}\right)$

$$
G_{\mu \nu ; \delta \gamma}^{\ell} d_{\ell}^{\delta \gamma}=\lambda_{\mu \nu}^{\ell} d_{\ell}^{\mu \nu}
$$

${ }_{351}$ Substitution of the eigenvectors $d^{\mu \nu}$ into the gap equation then 352 results in

$$
\begin{gathered}
1=-\nu_{0} \lambda_{\mu \nu}^{\ell} L\left(T_{c}, \mu, \epsilon_{c}\right), \\
L\left(T_{c}, \mu, \epsilon_{c}\right)=\int_{0}^{\varepsilon_{c}} \frac{d \varepsilon \varepsilon / \mu}{2|\varepsilon-\mu|} \tanh \left(\frac{|\varepsilon-\mu|}{2 T_{c}}\right), \quad \nu_{0}=\frac{N \mu}{2 \pi v^{2}},
\end{gathered}
$$

353 where $\nu_{0}$ is the density of states at the Fermi level, and $\varepsilon_{c}$ is an 354 ultraviolet cut-off. The logarithmic behavior of $L\left(T_{c}, \mu, \epsilon_{c}\right)$ gives rise 355 to the exponential dependence of $T_{c} \sim \varepsilon_{c} e^{-1 /\left(\nu_{0} \lambda_{\mu \nu}^{\ell}\right)}$ on the density ${ }_{356}$ of states $\nu_{0}$ and the eigenvalue $\lambda_{\mu \nu}$, which must be negative for the 357 the gap equation to have a solution, corresponding to an attractive 358 interaction. 
359 Using the explicit form of the interactions (12) and (13), we find 396 additional cosine terms which respect the honeycomb symmetry, as 360 the three dominant gap structures, with the following (negative) 397 discussed in [49]; (ii) corrections to the infinite square well potential

361 eigenvalues of $G(17)$,

$$
\begin{aligned}
d_{\ell= \pm 1}^{x \mp}: & \lambda_{x \pm}^{\mp 1}=g_{0}+g_{1}+g_{2}+g_{3}-g_{4}-g_{5}, \\
d_{\ell= \pm 1}^{z z}: & \lambda_{z z}^{ \pm 1}=g_{0}-g_{1}-g_{2}+g_{3}+j_{0}+j_{1}, \\
d_{\ell=0}^{z 0}: & \lambda_{z 0}^{0}=\tilde{g}_{0}-\tilde{g}_{1}-\tilde{g}_{2}+\tilde{g}_{2}-\tilde{j}_{0}-\tilde{j}_{1} .
\end{aligned}
$$
364 ence to these eigenvalues (20a)-(20c). We focus on terms that do not 365 contain $s_{z}$ in $(12)$ and (13), since these drive the transition, while $s_{z}$ 366 dependent terms act to fix the spin orientation of the corresponding 367 spin-triplet states.

368 For $\ell=0$, we identify the driving term for superconductivity as 369 $\tilde{j}_{0}\left(\tau^{x} \otimes \tau^{x}+\tau^{y} \otimes \tau^{y}\right)$, whereas for $\ell= \pm 1$, the driving term for 370 superconductivity is $g_{0}\left(\tau_{0} \otimes \tau_{0}\right)$. The coupling $\tilde{j}_{0}$ is positive, and 371 antiscreening increases its magnitude as the chemical potential in372 creases. Hence choosing a valley singlet structure generates a nega373 tive eigenvalue $-\tilde{j}_{0}$, analogous to how antiferromagnetism promotes 374 spin singlet pairing. Antiscreening in $g_{0}$ manifests as a sign change $-{ }^{415}$ 375 for large enough chemical potential, $g_{0}$ is overscreened and becomes 376 negative, as has been previously discussed in Refs $[1,30]$.

\section{Explicit solution and phase diagram}

378

In this section we construct the phase diagram consisting of the ${ }_{419}$ 379 three leading superconducting instabilities of (20a)-(20c), as well as 420 380 for competing charge and magnetic order, which will be described in ${ }_{421}$ 381 Section VI.

382 We specify the phase diagram as follows: (a) we choose to fix ${ }_{423}$ 383 the ratio $d / L=0.375$, which as we have stated earlier quantifies ${ }_{424}$ 384 the strength of spin-orbit coupling, with a physically achievable ${ }_{425}$ ${ }_{385} L=30 \mathrm{~nm}$. Other (similar) values of $d / L$ will act to affect the 386 phase boundaries, but not the phases themselves. (b) We desig387 nate a critical doping $\mu_{c}=0.025 v K_{0}$ and plot phase diagrams for ${ }_{388} \mu / \mu_{c}=\{0.75,1,1,25\}$. (c) We use the magnitude of the bare inter389 action matrix elements, $u_{\mu \nu}, v_{\mu \nu}$ - i.e. $v_{12}, v_{33}, u_{00}, u_{12}$ from $(5)-$ as ${ }_{427}$ 390 continuous tuning parameters. As shown in Fig. 4, we plot two sets ${ }_{428}$ 391 of diagrams spanned by $\left(\tilde{v}_{12} / v_{12}, \tilde{u}_{12} / u_{12}\right)$ and $\left(\tilde{v}_{33} / v_{33}, \tilde{u}_{00} / u_{00}\right) . \quad{ }_{429}$ 392 The motivation for choice $(c)$ is that one expects quantitative ${ }_{430}$ 393 changes to the values of bare interaction matrix elements (5), shown ${ }_{431}$ 394 in Fig. 3, for four reasons: $(i)$ inaccuracies of the microscopic mod395 eling, such as those due to neglecting higher harmonics in (3), i.e. 398 (2); $($ iii $)$ corrections of order $W_{0} / E_{0}$, which are not captured in the 399 three $K$-point approach; finally $(i v)$ since we only present results for 400 a InAs heterostructure, the variation in the calculated bare values 401 may be very approximately linked to teasing out the phase diagram 402 for other choices of semiconductor heterostructures. Hence, instead 403 of incorporating all such corrections numerically, we will allow the 404 bare interaction parameters to vary about the values presented in 405 Fig. 3. In this way we absorb uncertainty due to microscopic details 406 of the superlattice potential into the numerical values of the bare ${ }_{07}$ interaction parameters $v_{i j}, u_{i j}(5)$.

${ }_{408}$ The dominant bare interactions are found to be $v_{12}, v_{33}, u_{00}, u_{12}$, 409 as shown in Figure 3, and for the purposes of presentation, we choose 40 to vary these four parameters. Denoting the freely varying parame${ }_{411}$ ters as $\tilde{v}_{12}, \tilde{v}_{33}, \tilde{u}_{00}, \tilde{u}_{12}$, in Figure 4 , we plot two sets of spanned by ${ }_{412}\left(\tilde{v}_{12} / v_{12}, \tilde{u}_{12} / u_{12}\right)$ and $\left(\tilde{v}_{33} / v_{33}, \tilde{u}_{00} / u_{00}\right)$.

${ }_{413}$ As anticipated in (20a),(20b),(20c), three distinct gap structures 14 appear in the phase diagram, which we describe here:

- Intravalley $\boldsymbol{p}+\boldsymbol{i} \tau \boldsymbol{p}$ spin-triplet, valley-triplet,

$$
\Delta_{\boldsymbol{k}}=e^{i \tau_{z}\left(\phi-\theta_{\boldsymbol{k}}\right)}\left(d_{s}^{x} s_{x}+d_{s}^{y} s_{y}\right) \tau_{y}\left(\tau_{y} s_{y}\right)
$$
1

2

The spin triplet vector is pinned in-plane, and the valley polarisation is coupled to the orbital angular momentum, i.e. $\ell= \pm 1$ at valley $\tau=\mp 1$. This implies a chiral $p$-wave gap, with opposite chiralities in each valley, a state which respects time reversal symmetry. This phase exhibits a $U(1) \times U(1)$ symmetry breaking due to the presence of a relative phase $\phi$ between opposite valleys and a spin direction $\boldsymbol{d}_{s}=\left(d_{s}^{x}, d_{s}^{y}, 0\right)$. The superconducting state is analogous to that of Ref. [30] but with $\boldsymbol{d}$ pinned in-plane. This state exhibits higher-order topology, as will be demonstrated in Section VII.

- Intervalley $\boldsymbol{p}+\boldsymbol{i} \boldsymbol{p}$ spin-triplet, valley-triplet,

$$
\Delta_{\boldsymbol{k}}=e^{ \pm i \theta_{\boldsymbol{k}}} d_{s}^{z} s_{z} \tau_{z}\left(\tau_{y} s_{y}\right)
$$

Here the chiral angular momentum states $\ell= \pm 1$ are degenerate. An analysis of the Landau-Ginzburg free energy is required to understand if these degenerate states compete or coexist. A simple computation gives the Landau-Ginzburg free energy for the two order parameters $e^{ \pm i \theta_{k}} s_{z} \tau_{z} \equiv \phi_{ \pm}$,

$$
\mathcal{F}\left[\phi_{ \pm}\right]=-s\left(\phi_{+}^{2}+\phi_{-}^{2}\right)+\alpha\left(\phi_{+}^{4}+4 \phi_{+}^{2} \phi_{-}^{2}+\phi_{-}^{4}\right)
$$


(ai)

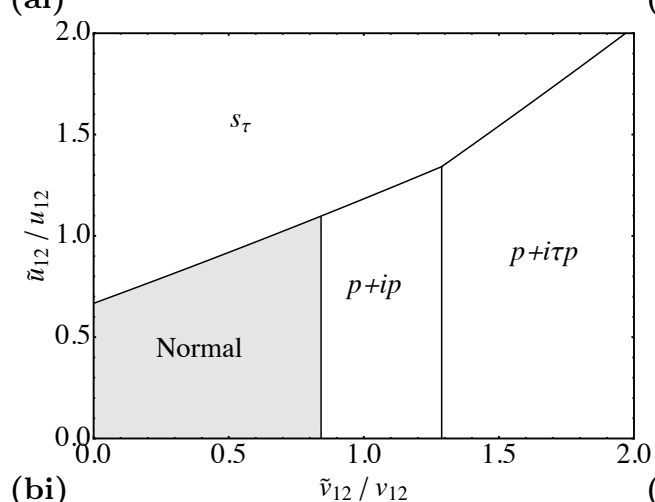

(bi)

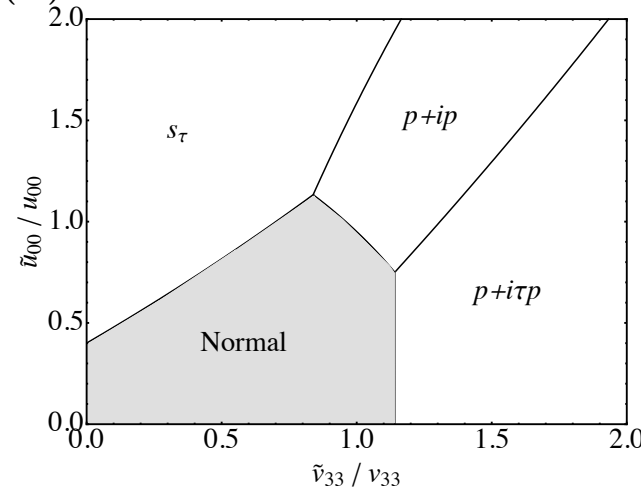

(aii)

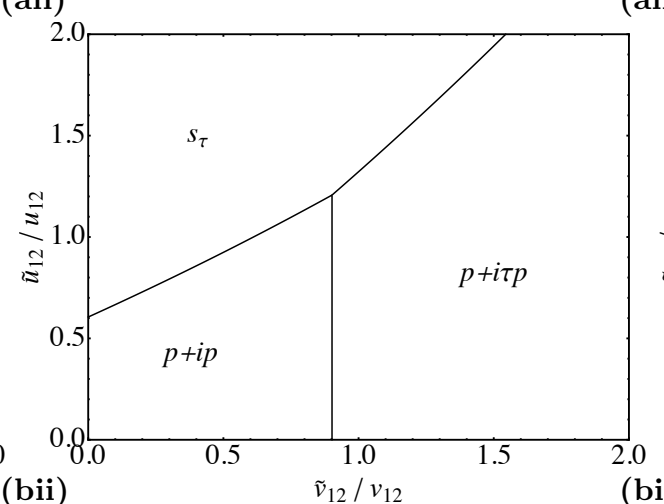

(aiii)

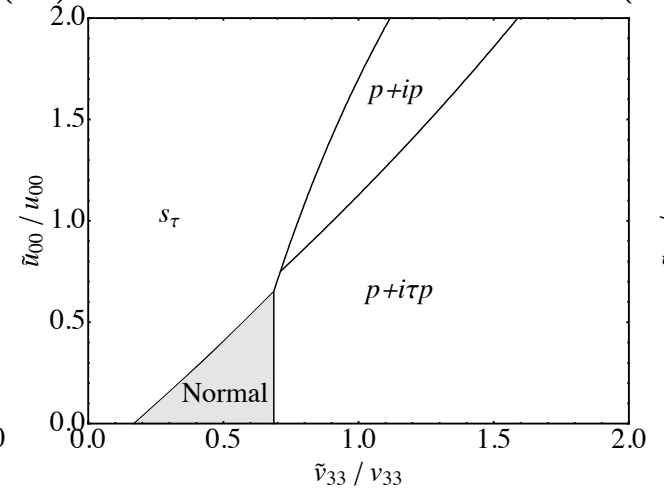

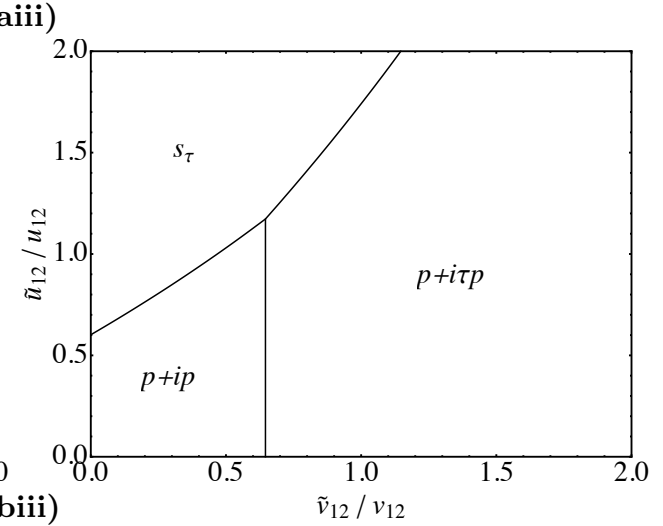

(bii)

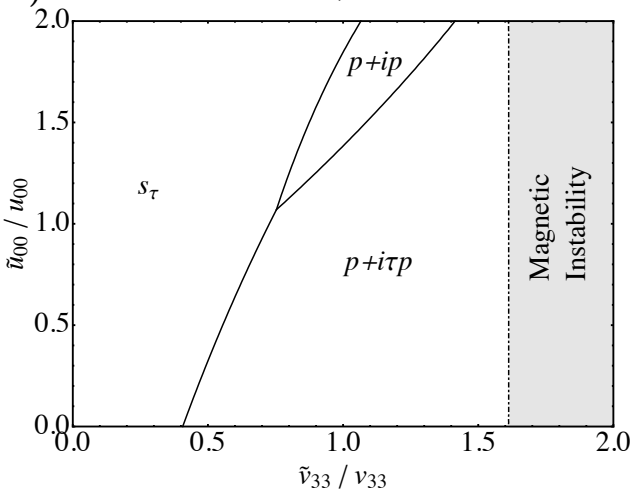

FIG. 4: Phase diagram. The three superconducting phases $d_{z 0}^{\ell=0}, d_{z z}^{\ell= \pm}, d_{\ell= \pm}^{\alpha \pm}$, which correspond to $s$-wave intervalley $\left(s_{\tau}\right), p+i p$-wave intervalley $(p+i p)$, and $p+i \tau p$ intravalley $(p+i \tau p)$, as well as the magnetic phase. (a) Fixing the bare values

$\left\{v_{00}, v_{33}, v_{44}, v_{77}, v_{56}, v_{07}, v_{47}, u_{00}, u_{33}, u_{03}\right\}$ to those computed and shown in Figure 3, while allowing for a variable $\tilde{v}_{12}$ and $\tilde{u}_{12}$, which are substituted into the interaction structure in place of $v_{12}$ and $u_{12}$. Here we show the variable values as ratio of the calculated values. (ai), (aii), (aiii) Show the same parameters but with increasing chemical potential $\mu / \mu_{0}=0.75,1,1.25$, respectively, with $\mu_{0} \equiv 0.025 v K_{0}$, with $d / L=0.375, L=30 \mathrm{~nm}$. (b) Same as (a), but allowing for a variable $\tilde{v}_{33}$ and $\tilde{u}_{00}$. (bi), (bii), (biii) Show the same parameters but with increasing chemical potential $\mu / \mu_{0}=0.75,1,1.25$.

The quartic term breaks the $S O(2)$ rotational symmetry in ${ }_{444}$ the isospin space $\left(\phi_{+}, \phi_{-}\right)$, and the order parameters $\phi_{ \pm}$act ${ }_{445}$ like an Ising degree of freedom; the system must spontaneously ${ }_{446}$ choose a chirality $(\ell= \pm 1)$, and therefore spontaneously break time reversal symmetry. This phase possesses a nontrivial firstorder topological invariant which manifests as chiral modes propagating along the edge, as we discuss in Section VII.

- Intervalley $\boldsymbol{s}_{\tau}$ spin-triplet, valley-singlet,

$$
\Delta_{\boldsymbol{k}}=d_{s}^{z} s_{z} \tau_{0}\left(\tau_{y} s_{y}\right)
$$

(24) ${ }_{453}$ Note that the phase boundaries between normal and supercon${ }_{454}$ ducting states are second-order, while the phase boundaries between The spin triplet vector is pinned out-of-plane along $\boldsymbol{z}$. As 455 distinct superconducting states are first-order, which in principle shown in [106], owing to the valley singlet structure, this spin ${ }_{456}$ leaves open the possibility of coexistence between these supercontriplet phase satisfies an "Anderson theorem", which provides 457 ducting phases. However, a straightforward Landau-Ginsburg analprotection against non-magnetic disorder, provided the disor- 458 ysis shows that all coexistence is energetically penalised.

der does not induces intervalley scattering. Quite unexpectedly, this phase hosts a second-order topological invariant, to be described in Section VII.

${ }_{447}$ As can be seen in Figure 4, for each superconducting state there is ${ }_{448}$ a critical $\mu_{c}$ such that for $\mu>\mu_{c}$ the system become superconduct${ }_{449} \mathrm{ing}$, which as discussed earlier reflects the fact that as the chemical 450 potential is increased, screening becomes more efficient, causing the ${ }_{451}$ pseudospin and/or valley dependent interactions to become attrac452 tive. le 
459 Finally, the rightmost portion of Figure 4 contains a region la- 476 diverge, a condition which results in equations analogous to 460 belled as a "magnetic instability". In this region, we find that mag- 477 the BCS gap equation. Denoting the particle-hole susceptibility ${ }_{461}$ netic insulating states can compete with superconductivity, as we ${ }_{478} \chi_{s_{1} \tau_{1}, s_{2} \tau_{2}}\left(p_{0}, \boldsymbol{p}\right)$, FM order corresponds to a divergence in $\chi_{1}=$ 462 will discuss in Section VI. $479 \chi_{s \tau,-s \tau}(0, \mathbf{0})$, SDW order to $\chi_{2}=\chi_{s \tau,-s-\tau}(0, \mathbf{0})$ and CDW to ${ }_{480} \chi_{3}=\chi_{s \tau, s-\tau}(0, \mathbf{0})$.

\section{COMPETING INSTABILITIES}

464 In this section we address the question of whether other instabili465 ties may compete with superconductivity. As is expected in general, ${ }_{466}$ superconductivity dominates in the limit of weak interactions when ${ }_{467}$ the Fermi surface is not nested. We find a portion of the phase di468 agram at stronger couplings in which magnetic order dominates, as 469 shown in Figure 4.

470 We consider three types of instabilities: ferromagnetism (FM), ${ }_{471}$ spin density wave (SDW), and charge density wave (CDW), where ${ }_{472}$ the SDW and CDW states are commensurate with the lattice with ${ }_{473}$ period $\boldsymbol{K}$. These phases are referred to as particle-hole instabilities, 474 and are captured by the order parameters, respectively

$$
\begin{aligned}
& \Phi_{1}=\sum_{\boldsymbol{k}} \psi_{\uparrow, \tau, \boldsymbol{k}}^{\dagger} \psi_{\downarrow, \tau, \boldsymbol{k}}, \\
& \Phi_{2}=\sum_{\boldsymbol{k}} \psi_{\uparrow, \tau, \boldsymbol{k}}^{\dagger} \psi_{\downarrow,-\tau, \boldsymbol{k}}, \\
& \Phi_{3}=\sum_{\boldsymbol{k}} \psi_{\uparrow, \tau, \boldsymbol{k}}^{\dagger} \psi_{\uparrow,-\tau, \boldsymbol{k}} .
\end{aligned}
$$

These orders take hold when their associated susceptibilities
${ }_{481}$ In the previous section, we wrote the interactions in the basis of ${ }_{482}$ states in the upper band (ie at the Fermi surface) with $\boldsymbol{k}_{1}=-\boldsymbol{k}_{3}$, ${ }_{483} \boldsymbol{k}_{2}=-\boldsymbol{k}_{4}$, corresponding to the Cooper scattering channel. For the ${ }_{484}$ particle-hole instabilities, the relevant scattering amplitudes are the 485 direct and exchange channels. We again restrict all momenta to lie ${ }_{486}$ on the Fermi surface $\left|\boldsymbol{k}_{i}\right|=k_{F}$. The scattering condition for the ${ }_{487}$ exchange channel is $\boldsymbol{k}_{1}=\boldsymbol{k}_{4}, \boldsymbol{k}_{2}=\boldsymbol{k}_{3}$, with scattering angle $\theta \equiv$ ${ }_{488} \theta_{k_{2}}-\theta_{k_{1}}$. The corresponding couplings $\mathcal{V}$ are obtained analogously 489 to the procedure in the Cooper channel (C3). We further define the ${ }_{490} \ell=0$ component as $\int d \theta \hat{V} /(2 \pi) \equiv \hat{\mathcal{V}}^{X}$. For the density channel, ${ }_{491} \boldsymbol{k}_{1}=\boldsymbol{k}_{2}, \boldsymbol{k}_{3}=\boldsymbol{k}_{4}$, with scattering angle $\theta \equiv \theta_{k_{3}}-\theta_{k_{1}}$, and we define ${ }_{492}$ the $\ell=0$ component as $\int d \theta \hat{V} /(2 \pi) \equiv \hat{\mathcal{V}}^{D}$. The procedure of the 493 previous section yields the tensor,

494 The ladder equations give the total susceptibilities in terms of the ${ }_{495}$ static susceptibilities and the direct/exchange scattering amplitudes,

$$
\begin{aligned}
\chi_{s_{1} \tau_{1}, s_{2} \tau_{2}}(0, \mathbf{0}) & =\chi_{s_{1} \tau_{1}, s_{2} \tau_{2}}^{0}(0, \mathbf{0})+\sum_{s_{a}, s_{b}, \tau_{a}, \tau_{b}} \int \frac{d^{3} q_{1} d^{3} q_{2}}{(2 \pi)^{6}} G_{s_{1} \tau_{1}}\left(q_{10}, \boldsymbol{q}_{\mathbf{1}}\right) G_{s_{2} \tau_{2}}\left(q_{10}, \boldsymbol{q}_{\mathbf{1}}\right) V_{s_{1} \tau_{1} s_{a} \tau_{a}, s_{2} \tau_{2} s_{b} \tau_{b}}\left(\theta_{2}-\theta_{1}\right) G_{s_{a} \tau_{b}}\left(q_{20}, \boldsymbol{q}_{\mathbf{2}}\right) G_{s_{b} \tau_{b}}\left(q_{20}, \boldsymbol{q}_{\mathbf{2}}\right)+\ldots \\
& =\chi_{s_{1} \tau_{1}, s_{2} \tau_{2}}^{0}(0, \mathbf{0})+\chi_{s_{1} \tau_{1}, s_{2} \tau_{2}}^{0}(0, \mathbf{0}) \sum_{s_{a}, s_{b}, \tau_{a}, \tau_{b}}\left(\int \frac{d \theta}{2 \pi} V_{s_{1} \tau_{1} s_{a} \tau_{a}, s_{2} \tau_{2} s_{b} \tau_{b}}\left(\theta_{2}-\theta_{1}\right)\right) \chi_{s_{a} \tau_{a}, s_{b} \tau_{b}}(0, \mathbf{0}) \\
& =\chi_{s_{1} \tau_{1}, s_{2} \tau_{2}}^{0}(0, \mathbf{0})+\chi_{s_{1} \tau_{1}, s_{2} \tau_{2}}^{0}(0, \mathbf{0}) \sum_{s_{a}, s_{b}, \tau_{a}, \tau_{b}} \mathcal{V}_{s_{1} \tau_{1} s_{a} \tau_{a}, s_{2} \tau_{2} s_{b} \tau_{b}}^{X / D} \chi_{s_{a} \tau_{a}, s_{b} \tau_{b}}(0, \mathbf{0})
\end{aligned}
$$

496 where the static susceptibilities are given by

$$
\chi_{s_{1} \tau_{1}, s_{2} \tau_{2}}^{0}(0, \mathbf{0}) \equiv-i \int \frac{d^{3} q}{(2 \pi)^{3}} G_{s_{1} \tau_{1}}\left(q_{0}, \boldsymbol{q}\right) G_{s_{2} \tau_{2}}\left(q_{0}, \boldsymbol{q}\right) .
$$

${ }_{497}$ For FM, SDW, and CDW order, we evaluate the relevant static 498 susceptibilities as $\chi_{s \tau,-s \tau}^{0}\left(q_{0}=0, \boldsymbol{q}=0\right)=\chi_{s \tau,-s-\tau}^{0}\left(q_{0}=0, \boldsymbol{q}=\right.$ $\left.{ }_{499} 0\right)=\chi_{s \tau, s-\tau}^{0}\left(q_{0}=0, \boldsymbol{q}=0\right)=\mu /(2 \pi)$. The resulting RPA equations ${ }_{501}$
500 for the susceptibilities reduce to

$$
\begin{aligned}
& \chi_{1}=\frac{\mu}{2 \pi}+\frac{\mu}{2 \pi}\left(\hat{\mathcal{V}}_{s+s+, \bar{s}+\bar{s}+}^{X}+\hat{\mathcal{V}}_{s+s-\bar{s}-\bar{s}+}^{X}\right) \chi_{1}, \\
& \chi_{2}=\frac{\mu}{2 \pi}+\frac{\mu}{2 \pi}\left(\hat{\mathcal{V}}_{s+s+, \bar{s}-\bar{s}-}^{X}\right) \chi_{2}, \\
& \chi_{3}=\frac{\mu}{2 \pi}+\frac{\mu}{2 \pi}\left(\hat{\mathcal{V}}_{s+s-, s-s+}^{D}\right) \chi_{3} .
\end{aligned}
$$

The condition for FM, SDW and CDW instabilities immediately 
502 follow, and can be written analytically in terms of the coupling con503 stants found in (26),

$$
\begin{aligned}
(\mathrm{FM}): 1 & =\frac{\mu}{2 \pi}\left(\hat{\mathcal{V}}_{s+s+, \bar{s}+\bar{s}+}^{X}+\hat{\mathcal{V}}_{s+s-, \bar{s}-\bar{s}+}^{X}\right) \\
& =\frac{\mu}{2 \pi}\left(g_{0}^{X}+g_{1}^{X}-g_{2}^{X}-g_{3}^{X}+j_{0}^{X}-j_{1}^{X}\right) \\
(\mathrm{SDW}): 1 & =\frac{\mu}{2 \pi} \hat{\mathcal{V}}_{s+s+, \bar{s}-\bar{s}-}^{X}=\frac{\mu}{2 \pi}\left(g_{0}^{X}-g_{1}^{X}-g_{2}^{X}+g_{3}\right) \\
(\mathrm{CDW}): 1 & =\frac{\mu}{2 \pi} \hat{\mathcal{V}}_{s+s-, s-s+}^{D}=\frac{\mu}{2 \pi} j_{0}^{D} .
\end{aligned}
$$

504 From Eq. (29), the system exhibits instabilities which compete 505 with superconductivity when the dimensionless coupling constants 506 are order unity - as distinct from the superconducting instability 507 which occurs for arbitrarily weak attractive coupling. Throughout 508 most of the phase diagram we considered, this condition is not met, 509 and so superconductivity is the sole instability of the system.

${ }_{510}$ Increasing the chemical potential, the antiscreening mechanism 511 causes the $g_{0}$ coupling to grow large. Since this coupling does not ${ }_{512}$ appear in the susceptibility for CDW order, antiscreening gives rise 513 to only FM and SDW ordering. We do find one small region of phase 514 diagram where $g_{0}$ grows large enough to give rise to FM and SDW 515 order. The other couplings, which are much smaller than $g_{0}$, act to ${ }_{516}$ favor SDW over FM order. In the phase diagram we simply label 517 this region as magnetic instability since we expect FM and SDW ${ }_{518}$ to be nearly degenerate. Moreover, this part of the phase diagram 519 should not be taken too literally, since the large coupling constant ${ }_{520}$ means corrections to mean field theory are likely significant.

\section{TOPOLOGICAL PROPERTIES OF THE SUPERCONDUCTING PHASES}

${ }_{523}$ In this section we will prove that all three superconducting phases ${ }_{524}$ are topological, and discuss their properties. For intervalley pairing, 525 we have $\Delta_{k} \propto \tau_{y}$ and $\Delta_{k} \propto e^{ \pm i \theta_{k}} \tau_{x}$ for the $s_{\tau}$ and $p+i p$ phases 526 respectively, while $\Delta_{\boldsymbol{k}} \propto e^{i \tau_{z}\left(\phi-\theta_{\boldsymbol{k}}\right)}$ for the intravalley $p+i \tau p$ phase. ${ }_{527}$ Since $\tau_{x}, \hat{\tau}_{z}$ are even under inversion $(\boldsymbol{r} \rightarrow-\boldsymbol{r})$ while $\tau_{y}$ is odd, we ${ }_{528}$ find that the gap is odd under inversion for both intervalley phases, ${ }_{529}$ while the intravalley $p+i \tau p$ phase is even for $\phi=n \pi$ and odd 530 for $\phi=\left(n+\frac{1}{2}\right) \pi$, with $n \in \mathbb{Z}$. A fundamental requirement for a 531 non trivial topology hosting Majorana edge or corner modes is that 532 the gap change sign under inversion ${ }^{1}$. This is fulfilled for both the

1 A close examination of the classification presented in Refs. [4, 8] reveals that when the system respects time-reversal symmetry, i.e. in Cartan class DIII,
${ }_{33}$ intervalley phases, as well as for the intravalley $p+i \tau p$ phase in the ${ }_{534}$ special case $\phi=\left(n+\frac{1}{2}\right) \pi$.

${ }_{535}$ The time-reversal symmetry breaking intervalley $p+i p$ phase 536 exhibits first-order topology; taking into account the $U(1)$ spin${ }_{537}$ rotation symmetry, we find that this system is in Cartan class A, ${ }_{538}$ which permits a Chern number in two dimensions [107, 108]. We ${ }_{539}$ find that this phase exhibits a pair of chiral Dirac modes propagat540 ing along the boundary, establishing it as a first-order topological ${ }_{541}$ superconductor.

${ }_{542}$ The intervalley $s_{\tau}$ phase is time-reversal symmetric, and account${ }_{54}$ ing for the $U(1)$ spin-rotation symmetry, is in class AIII, which 544 always implies trivial first-order topology in two dimensions. The ${ }_{545}$ time-reversal symmetric intravalley $p+i \tau p$ satisfies a $\mathbb{Z}_{2}$ symmetry 546 expressed by a combination of spin rotation and gauge transforma547 tion, such that the system is described by a BdG Hamiltonian in ${ }_{548}$ class D. For intervalley $s_{\tau}$ and intravalley $p+i \tau p$, a second-order 549 topological phase protected by the crystalline symmetries is possible. ${ }_{550}$ We will establish the second-order topology for intravalley $p+i \tau p$ 551 and intervalley $s_{\tau}$ pairing using symmetry-based indicators. Finally, 552 we will present exact diagonalisation results for the Bogoliubov-de ${ }_{553}$ Gennes Hamiltonian for all three superconducting phases. These nu554 merical results provide clear evidence for the suggested topology by 555 demonstrating the corresponding anomalous edge and corner states. 556 It is first necessary to express the mean field Hamiltonian (14) ${ }_{557}$ as a lattice model involving creation operators $c_{\boldsymbol{R}, s}^{\dagger}$ for Wannier or${ }_{558}$ bitals localised at the sites $\boldsymbol{R}$ of the artificial honeycomb lattice, ${ }_{559} \mathcal{H}_{\mathrm{MF}}=\mathcal{H}_{\text {nor. }}+\mathcal{H}_{\Delta}$. The normal state Hamiltonian $\mathcal{H}_{\text {nor. }}$ is equiva560 lent to two copies of the Haldane model, consisting of a sum of spin${ }_{561}$ independent nearest neightbour hoppings and next nearest neigh562 bour spin-dependent hopping terms,

$$
\mathcal{H}_{\text {nor. }}=-\sum_{\left\langle\boldsymbol{R}, \boldsymbol{R}^{\prime}\right\rangle ; s} t c_{\boldsymbol{R}, s}^{\dagger} c_{\boldsymbol{R}^{\prime}, s}-\sum_{\left\langle\left\langle\boldsymbol{R}, \boldsymbol{R}^{\prime}\right\rangle\right\rangle ; s} t^{\prime} e^{\frac{2 \pi i}{3} \sigma s} c_{\boldsymbol{R}, s}^{\dagger} c_{\boldsymbol{R}^{\prime}, s}
$$

563 where the parameters of the Dirac model (9) are related to the hop564 ping parameters via $v=\sqrt{3} a t / 2$ and $t^{\prime}=9 \eta / 2$.

and the gap is even under inversion, the topological classification with inversion symmetry is trivial. This implies that a first-order topological phase hosting a helical Majorana edge mode, as well as a second-order topological phase hosting Kramers pairs of Majorana corner state, is prohibited. When more symmetries are included it is still possible that further topological phases appear, however they must have distinct boundary signatures from the ones mentioned. 
The pairing term $H_{\Delta}$ is given by

$$
H_{\Delta}=\sum_{\boldsymbol{R}, \boldsymbol{R}^{\prime}} \Delta\left(\boldsymbol{R}, \boldsymbol{R}^{\prime}\right) c_{\boldsymbol{R}, \uparrow}^{\dagger} c_{\boldsymbol{R}, \downarrow}^{\dagger}
$$

566 for the intervalley $p+i p$ and $s_{\tau}$ phases with pairing between opposite 567 spins, and

$$
H_{\Delta}=\sum_{\boldsymbol{R}, \boldsymbol{R}^{\prime}} \Delta\left(\boldsymbol{R}, \boldsymbol{R}^{\prime}\right) \frac{1}{2}\left[e^{i \phi_{s}} c_{\boldsymbol{R}, \uparrow}^{\dagger} c_{\boldsymbol{R}^{\prime}, \uparrow}^{\dagger}+e^{-i \phi_{s}} c_{\boldsymbol{R}, \downarrow}^{\dagger} c_{\boldsymbol{R}^{\prime}, \downarrow}^{\dagger}\right]
$$

${ }_{568}$ for the intravalley $p+i \tau p$ phase with equal spin pairing, where ${ }_{569}\left(d_{x}, d_{y}, d_{z}\right)=\left(\sin \phi_{s}, \cos \phi_{s}, 0\right)$. The form of the pairing function ${ }_{570} \Delta\left(\boldsymbol{R}, \boldsymbol{R}^{\prime}\right)$ may be derived by projecting the momentum-space ex${ }_{571}$ pression for $H_{\Delta}$ in (14) onto the Wannier orbitals, and are derived 572 in the Appendix. For the intervalley paired phases, $\Delta\left(\boldsymbol{R}, \boldsymbol{R}^{\prime}\right)$ pos- 610 ${ }_{573}$ sesses the discrete translational symmetry of the lattice and changes 574 sign under inversion, $\Delta\left(-\boldsymbol{R},-\boldsymbol{R}^{\prime}\right)=-\Delta\left(\boldsymbol{R}, \boldsymbol{R}^{\prime}\right)$, while for the in- 6 575 travalley $p+i \tau p$ phase, the discrete translation symmetry of the 576 lattice is spontaneously broken and $\Delta\left(\boldsymbol{R}, \boldsymbol{R}^{\prime}\right)$ exhibits spatial mod577 ulations, oscillating as a function of $\boldsymbol{R}+\boldsymbol{R}^{\prime}$ and, except at special 578 values $\phi=n \pi / 2$, also spontaneously breaks inversion symmetry.

\section{A. Symmetry-based indicators for $p+i \tau p$ and $s_{\tau}$ phases}

${ }_{580}$ In this subsection, we prove that the superconducting states with ${ }_{581} p+i \tau p$ or $s_{\tau}$ pairing symmetry realise a second-order topological ${ }_{582}$ phase with Majorana Kramers pairs pinned to the corners by the ${ }_{583}$ crystalline point-group symmetries. We will first examine the sym584 metries of the system to determine under which conditions we may 585 expect a second-order topological phase. Next, we apply the the586 ory of symmetry-based indicators [5-7] to derive a simple, sufficient ${ }_{587}$ criterion for a transition into a second-order topological supercon${ }_{588}$ ducting state when an infinitesimal pairing which is odd under in589 version symmetry creates a full gap in the BdG spectrum. Finally, ${ }_{590}$ we show that this criterion is fulfilled for the $p+i \tau p$ and $s_{\tau}$ pairing 591 instabilities in our honeycomb lattice model.

${ }_{592}$ The symmetry-group of our hexagonal lattice is given by the di593 rect product of translations in the $x, y$ plane and the crystalline point 594 group $D_{6 h} \simeq C_{6 v} \otimes C_{i}$, where $C_{i}$ is generated by spatial inversion ${ }_{595} \mathcal{I}: x, y, z \rightarrow-x,-y,-z$ and $C_{6 v}$ is the point group of the hexagonal 596 lattice in the $x, y$ plane. Furthermore, the normal-state Hamiltonian ${ }_{597}$ satisfies time-reversal symmetry $\mathcal{T}$ and $U(1)$ spin rotation symmetry ${ }_{598} \mathcal{S}$ around the $s_{z}$ axis. A symmetric unit cell can be chosen to co599 incide with the hexagons in the hexagonal lattice, where the lattice 600 sites are located on the threefold rotation symmetric corners of the
601 hexagonal unit cell. Each site is occupied by one Kramers pair of 602 fermionic orbitals, which, without loss of generality for the following 603 discussion, can be chosen to be $s$-orbitals ${ }^{2}$. In the following, we ar${ }_{604}$ gue that inversion symmetry is sufficient to protect the second-order 605 topological phase and prove its appearance from the symmetry-based 606 indicator. Therefore, it is sufficient to consider the representations ${ }_{607}$ of time-reversal symmetry and inversion symmetry. In Bloch basis, 608 these representations in the normal state can be written as

$$
\begin{aligned}
u(\mathcal{T}) & =i s_{y} \sigma_{0} \\
u(\mathcal{I} ; \boldsymbol{k}) & =s_{0} \sigma_{x} e^{i\left(\boldsymbol{a}_{2}-\boldsymbol{a}_{1}\right) \boldsymbol{k}}
\end{aligned}
$$

with $s_{i}, \sigma_{i}$ the Pauli matrices in spin and sublattice space, re${ }_{610}$ spectively, and the Bravais lattice vectors $\boldsymbol{a}_{1}=\sqrt{3} a \hat{x}, \boldsymbol{a}_{2}=$ $611(\sqrt{3} a \hat{x}+3 a \hat{y}) / 2$, where $a$ is the interatomic distance. Here we chose 612 the center of the hexagons as the center of inversion.

${ }_{613}$ The $p+i \tau p$ and $s_{\tau}$ superconducting orders preserve time-reversal 614 symmetry. Out of the large symmetry-group containing the point ${ }_{615}$ group $D_{6 h}$ and $\mathbb{Z}_{2}$ or $U(1)$ spin rotation symmetry, respectively, it ${ }_{616}$ is sufficient to preserve only a single crystalline symmetry element ${ }_{617}$ such as inversion, perpendicular twofold rotation, or mirror symme${ }_{618}$ try in order to protect a second-order topological phase $[4,8]$. Here, 619 we focus on inversion symmetry, as it also allows us to write down a 620 symmetry-based indicator as a topological invariant. By restricting ${ }_{621}$ the topological classification to inversion and time-reversal symmetry 622 and neglecting the remaining symmetries, we resolve the topological ${ }_{623}$ phases in Cartan class DIII with inversion symmetry ${ }^{3}$. The remain${ }_{624}$ ing symmetry elements apart from time-reversal and inversion may ${ }_{625}$ enrich these topological phases, either prohibiting or giving rise to ${ }_{626}$ further topological phases. For example, the $U(1)$ spin rotation sym${ }_{627}$ metry prohibits the first-order topological superconductor in Cartan ${ }_{628}$ class DIII with helical Majorana edge states. The mirror and sixfold ${ }_{629}$ rotation symmetry enrich the second-order topological phase pro630 tected by inversion, as the mirror symmetry pins the corner states

\footnotetext{
2 The $s$-orbitals are even under inversion. Choosing different orbitals may change the representation of inversion symmetry that is carried through the calculation, but does not affect the conclusions.

${ }^{3}$ Notice that previously, we took the $U(1)$ spin-rotation symmetry or $\mathbb{Z}_{2}$ combined spin-gauge symmetry into account to conclude that each of the spinblocks is in Cartan class AIII or D, respectively. Here, we only utilize a minimal set of symmetries that is necessary to protect the second-order topological phase whose existence we want to prove, which does not require additional $U(1)$ or $\mathbb{Z}_{2}$ symmetry. Thus we may utilize the results for the less restrictive class DIII.
} 
${ }_{631}$ to mirror-symmetric corners and at the same time requires a gap- 673 at the inversion symmetric momenta $\mathbf{k}_{s}=\Gamma, M$. Here, we used that 632 less anomalous edge state on mirror symmetric edges $[3,4]$, while 674 sixfold rotation symmetry relates the three $M$ points in the hexag${ }_{633}$ the sixfold rotation symmetry requires that on a sixfold symmetric 675 onal Brillouin zone, such that $\mathfrak{N}_{+}^{M}=\mathfrak{N}_{+}^{M_{1}}=\mathfrak{N}_{+}^{M_{2}}=\mathfrak{N}_{+}^{M_{3}}$. For ${ }_{634}$ sample, gapless states should exist on all six corners.

676 the symmetry-based indicator, $z_{2}=1,3$ corresponds to a first-order

${ }_{635}$ The topological classification depends on whether the supercon- 677 topological superconductor with a helical Majorana edge state, and ${ }_{636}$ ducting order parameter is even or odd under inversion; this parity $678 z_{2}=2$ corresponds to the second-order topological superconduc${ }_{637}$ determines the representation of inversion symmetry and its com- 679 tor. In the weak pairing limit of an infinitesimal order parameter ${ }_{638}$ mutation relations with the particle-hole antisymmetry of the BdG $680 \Delta \rightarrow 0$, we can express the symmetry-based indicator in terms of ${ }_{639}$ Hamiltonian [5, 7]. In case the superconducting order parameter is 681 the symmetry-data of the normal-state Hamiltonian only:

640 even under inversion, the topological classification is trivial [4, 8]. ${ }_{641}$ In case it is odd under inversion, the classification of topological ${ }_{642}$ phases with anomalous boundary states is $\mathbb{Z}_{4}$, where odd elements ${ }_{643}$ "1", "3" indicate a first-order topological superconductor hosting a 682 where $\left.n_{ \pm}^{\mathbf{k}_{s}}\right|_{\text {occ }}$ ( $\left.n_{ \pm}^{\mathbf{k}_{s}}\right|_{\text {unocc }}$ ), are the occupied (unoccupied) Kramers ${ }_{644}$ helical Majorana edge mode, and the even element " 2 " is a second- 683 pairs of bands with inversion parity \pm 1 at the high-symmetry mo${ }_{645}$ order topological superconductor hosting Kramers pairs of Majorana 684 mentum $\mathbf{k}_{s}=\Gamma, M$. It is notable that this formula does not depend ${ }_{646}$ corner states on an inversion symmetric sample $[4,8]$.

685 on the properties of the low-energy theory at the $K, K^{\prime}$ points.

${ }_{647}$ The $p+i \tau p$-wave order parameter in Eq. (31) is spatially mod- ${ }_{686} s_{\tau}$ pairing. First, we evaluate the weak-pairing limit of the ${ }_{648}$ ulated $[1,30]$ such that it is even (odd) under inversion for $\phi=0{ }_{687}$ symmetry-based indicator for $s_{\tau}$-wave pairing. At the points $\Gamma, M$, ${ }_{649}(\pi / 2)$. For other values of $\phi$, the system does not respect inversion ${ }_{688}$ the energy of the bands is of the order of the nearest neighbour ${ }_{650}$ symmetry. Following the arguments above, this implies that we may 689 hopping $t$, which is our largest energy scale, $t \gg t^{\prime}, \mu, \Delta$. This al${ }_{651}$ find a second-order topological phase hosting Kramers pairs of Ma- 690 lows one to neglect spin-orbit coupling when computing the inversion ${ }_{652}$ jorana corner states only for $\phi=\pi / 2$. However, the corner states ${ }_{691}$ parities of the occupied and unoccupied bands. Without spin-orbit ${ }_{653}$ may persist for a range of $\phi$ around $\phi=\pi / 2$ until the surface gap ${ }_{692}$ coupling, the Bloch Hamiltonian for the nearest neighbour hopping ${ }_{654}$ closes $[1,3,4]$. The $s_{\tau}$-wave order parameter Eq. $(32)$ is odd under ${ }_{693}$ can be written as 655 inversion, thus allowing a second-order topological phase.

${ }_{656}$ Symmetry-based indicators are sufficient criteria for topological ${ }_{657}$ crystalline phases expressed in terms of symmetry-eigenvalues at a 658 few high-symmetry momenta only. A particular strength of this for659 malism is that in the weak-pairing limit of an infinitessimal pairing ${ }^{694}$ where we wrote the $2 \times 2$ matrix in sublattice space $\sigma$ explicitly. 660 strength $\Delta \rightarrow 0$, the symmetry-based indicator can be expressed 695 Together with the representation of inversion symmetry, Eq. 33, ${ }_{661}$ in terms of symmetry-data of the normal-state Hamiltonian only. ${ }_{696}$ we find by simultaneously diagonalising $h_{0}(\boldsymbol{k})$ and $u(\mathcal{I} ; \boldsymbol{k})$ for the ${ }_{662}$ The symmetry-based indicator takes the symmetry of the supercon- ${ }_{697}$ number of Kramers pairs resolved by their inversion parity $n_{+}^{\Gamma}$ occ $=$ ${ }_{663}$ ducting order parameter into account, as different symmetry-based $6980,\left.n_{-}^{\Gamma}\right|_{\text {unocc }}=0,\left.n_{+}^{M}\right|_{\text {occ }}=1,\left.n_{-}^{M}\right|_{\text {unocc }}=1$, such that $z_{2}^{\mathrm{WP}}=$ ${ }_{664}$ indicators are defined depending on the irreducible representation of 6992 . Taking into account that the $s_{\tau}$ pairing opens a full excitation 665 the order parameter. This allows one to formulate sufficient crite- 700 gap, the onset of this pairing instability is a second-order topological 666 ria for the topology of a superconducting phase depending on the 701 superconducting phase.

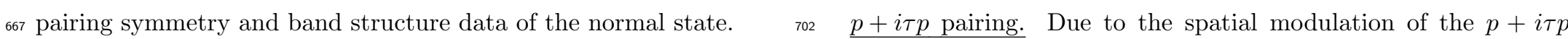
${ }_{668}$ The symmetry-based indicator for the second-order topolog- 703 superconducting order parameter, the Dirac cones at the $K$ and ${ }_{669}$ ical phase with inversion symmetry $\mathcal{I}$ and pairing symmetry $704 K^{\prime}$ points get folded onto the $\Gamma$ point. For finite hole doping, the $670 u(\mathcal{I}) \Delta\left(-k_{x},-k_{y}\right) u^{T}(\mathcal{I})=-\Delta\left(k_{x}, k_{y}\right)$ has been calculated as [5]

$$
z_{2}=\mathfrak{N}_{+}^{\Gamma}-\mathfrak{N}_{+}^{M} \bmod 4
$$

705 chemical potential lies inside the valence band. Taking the band 706 folding into account, we find $\left.n_{+}^{\Gamma}\right|_{\text {occ }}=0,\left.n_{-}^{\Gamma}\right|_{\text {unocc }}=2,\left.n_{+}^{M}\right|_{\text {occ }}=$ ${ }_{707} 2,\left.n_{-}^{M}\right|_{\text {unocc }}=2$ such that $z_{2}^{\mathrm{WP}}=2$. As the $p+i \tau p$ pairing instability ${ }_{671}$ where $\mathfrak{N}_{+}^{\mathbf{k}_{s}}$ is the number of Kramers pairs of eigenstates of the BdG 708 opens a full gap in the spectrum that is odd under inversion for ${ }_{672}$ Hamiltonian with negative energy and even inversion eigenvalue $+1709 \phi=\pi / 2$, it leads to a second-order topological phase for $\phi=\pi / 2$. 

712 employed a simplified lattice model which accounts only for pairing 713 between the closest sites for which the gap is nonvanishing. For the 714 intervalley $p+i p$ spin triplet phase

$$
\begin{gathered}
\mathcal{H}_{\Delta}=\sum_{\left\langle\boldsymbol{R}, \boldsymbol{R}^{\prime}\right\rangle} \Delta\left(\boldsymbol{R}, \boldsymbol{R}^{\prime}\right) c_{\boldsymbol{R}, \uparrow}^{\dagger} c_{\boldsymbol{R}^{\prime}, \downarrow}^{\dagger} \\
\Delta\left(\boldsymbol{R}, \boldsymbol{R}^{\prime}\right)= \begin{cases}\Delta^{\prime} e^{i\left(\theta-\frac{\pi}{2}\right)} & \boldsymbol{R}^{\prime} \in A \\
\Delta^{\prime} e^{i\left(\theta+\frac{\pi}{2}\right)} & \boldsymbol{R}^{\prime} \in B\end{cases}
\end{gathered}
$$

715 where $\theta$ is the hopping direction.

${ }_{716}$ For the intervalley $s_{\tau}$ spin triplet phase, we find that pairing van717 ishes exactly between nearest neighbours, thus we consider only pair718 ing between next nearest neighbours,

$$
\begin{gathered}
\mathcal{H}_{\Delta}=\sum_{\left\langle\left\langle\boldsymbol{R}, \boldsymbol{R}^{\prime}\right\rangle\right\rangle} \Delta\left(\boldsymbol{R}, \boldsymbol{R}^{\prime}\right) c_{\boldsymbol{R}, \uparrow}^{\dagger} c_{\boldsymbol{R}^{\prime}, \downarrow}^{\dagger} \\
\Delta\left(\boldsymbol{R}, \boldsymbol{R}^{\prime}\right)= \begin{cases}+\Delta^{\prime} & \theta=0, \pm \frac{2 \pi}{3} \\
-\Delta^{\prime} & \theta=\pi, \pm \frac{\pi}{3}\end{cases}
\end{gathered}
$$

${ }_{719}$ For the intravalley $p+i \tau p$ spin triplet phase, we consider pairing 720 between nearest neighbors,

$$
\begin{gathered}
\mathcal{H}_{\Delta}=\frac{1}{2} \sum_{\left\langle\boldsymbol{R}, \boldsymbol{R}^{\prime}\right\rangle} \Delta\left(\boldsymbol{R}, \boldsymbol{R}^{\prime}\right)\left(e^{i \phi_{s}} c_{\boldsymbol{R}, \uparrow}^{\dagger} c_{\boldsymbol{R}^{\prime}, \uparrow}^{\dagger}+e^{-i \phi_{s}} c_{\boldsymbol{R}, \downarrow}^{\dagger} c_{\boldsymbol{R}^{\prime}, \downarrow}^{\dagger}\right) \\
\Delta\left(\boldsymbol{R}, \boldsymbol{R}^{\prime}\right)= \begin{cases}+\Delta^{\prime} & \boldsymbol{R}^{\prime} \in A \\
-\Delta^{\prime} & \boldsymbol{R}^{\prime} \in B\end{cases}
\end{gathered}
$$

${ }_{721} \quad$ For the intervalley $s_{\tau}$ and $p+i p$ spin triplet phases, we may write 722 the Bogoliubov-de Gennes Hamiltonian in matrix form as

$$
\begin{aligned}
& \mathcal{H}_{\text {nor. }}+\mathcal{H}_{\Delta}= \\
& \frac{1}{2} \sum_{\boldsymbol{R}, \boldsymbol{R}^{\prime}}\left(\begin{array}{cc}
c_{\boldsymbol{R}, \uparrow}^{\dagger} & c_{\boldsymbol{R}, \downarrow}
\end{array}\right)\left(\begin{array}{cc}
H_{\uparrow \uparrow}\left(\boldsymbol{R}, \boldsymbol{R}^{\prime}\right) & \Delta_{\uparrow \downarrow}\left(\boldsymbol{R}, \boldsymbol{R}^{\prime}\right) \\
\Delta_{\uparrow \downarrow}^{\dagger}\left(\boldsymbol{R}, \boldsymbol{R}^{\prime}\right) & -H_{\downarrow \downarrow}^{*}\left(\boldsymbol{R}, \boldsymbol{R}^{\prime}\right)
\end{array}\right)\left(\begin{array}{c}
c_{\boldsymbol{R}^{\prime}, \uparrow} \\
c_{\boldsymbol{R}^{\prime}, \downarrow}^{\dagger}
\end{array}\right)
\end{aligned}
$$

725 for the intravalley $p+i \tau p$ spin-triplet phase as

$$
\begin{aligned}
& \mathcal{H}_{\text {nor. }}+\mathcal{H}_{\Delta}= \\
& \frac{1}{2} \sum_{\boldsymbol{R}, \boldsymbol{R}^{\prime}, s}\left(\begin{array}{ll}
c_{\boldsymbol{R}, s}^{\dagger} & c_{\boldsymbol{R}, s}
\end{array}\right)\left(\begin{array}{cc}
H_{s, s}\left(\boldsymbol{R}, \boldsymbol{R}^{\prime}\right) & \Delta_{s, s}\left(\boldsymbol{R}, \boldsymbol{R}^{\prime}\right) \\
\Delta_{s, s}^{\dagger}\left(\boldsymbol{R}, \boldsymbol{R}^{\prime}\right) & -H_{s, s}^{*}\left(\boldsymbol{R}, \boldsymbol{R}^{\prime}\right)
\end{array}\right)\left(\begin{array}{c}
c_{\boldsymbol{R}^{\prime}, s} \\
c_{\boldsymbol{R}^{\prime}, s}^{\dagger}
\end{array}\right)
\end{aligned}
$$

729 perform the exact diagonalisation in only one of the two spin blocks, 730 and infer the results in the other block by its relation required by

${ }_{732}$ We plot the spectrum of infinite superconducting ribbons in the ${ }_{733}$ intervalley $s_{\tau}$, intravalley $p+i \tau p$ and intervalley $p+i p$ spin triplet 734 phases in Figs. 5, 6, 8, as a function of momentum $k$ along the 735 ribbon respectively. We observe anomalous edge features in all three 736 cases. In most cases, the $1 \mathrm{D}$ dispersion of modes propagating along ${ }_{737}$ opposite edges is split, and the lines of different color and thickness 738 indicate opposite edge modes.

739 To demonstrate the second-order topology of the intervalley $s_{\tau}$ and 740 intravalley $p+i \tau p$ spin triplet phases, we show the wavefunction pro${ }_{741}$ file of the six lowest energy eigenstates forming the Majorana corner 742 modes on a hexagonal flake geometry, and corresponding spectrum 743 in Figs. 5 and 6. The exact diagonalisation of the BdG Hamiltoni744 ans was performing within a spin-block for both intervalley $s_{\tau}$ and 745 intravalley $p+i \tau p$ spin-triplet phases, c.f. Eqs. (40) and (41), so the 746 Majorana corner modes in both cases have a degenerate Kramers ${ }_{747}$ partner in the opposite spin block.

${ }_{748}$ For the intervalley $s_{\tau}$ spin triplet phase, in which pairing occurs 749 between opposite spins, we plot the spectrum of the non-redundant 750 BdG Hamiltonian, so that each energy eigenvalue corresponds to a 751 quasiparticle whose antiparticle is identical to its Kramers partner. 752 Decomposing each zero energy mode into two Majorana modes, we 753 find one Majorana Kramers pair at each corner which are protected 754 by time-reversal symmetry. Two gapless counterpropagating modes 755 are observed on each edge for the armchair geometry, but we find 756 no edge states for the zigzag geometry, as shown in Fig. 5. On the 757 flake geometry, Majorana corner states appear on corners between 758 zigzag edges. The Majorana corner modes are a signature of the in759 trinsic second-order topology of the crystalline bulk superconductor, 760 because these corner modes can not be removed without breaking 761 the symmetries or closing the bulk gap [4].

${ }_{762}$ For the intravalley $p+i \tau p$ pahse, in which pairing occurs for equal 763 spins, we plot the spectrum for the BdG Hamiltonian within a single 764 spin block, so that each energy eigenvalue corresponds to a quasi${ }_{765}$ particle with a Kramers partner in the opposite spin block. For a 766 hexagonal flake with an armchair boundary, at $\phi=\pi / 2$, we find ${ }_{767}$ one Majorana Kramers pair at each corner of the flake, protected 768 by time-reversal symmetry. These results confirm the existence of ${ }_{726}$ Here the two blocks with opposite $s_{z}$ eigenvalue are related by ${ }_{769}$ a second-order topology which we concluded in the previous section 727 time-reversal symmetry $\mathcal{T}=i s_{y} K$, while each block separately sat- 770 via the symmetry-based indicators. We observe gapless counterprop728 isfies particle-hole symmetry. The block-diagonal form allows us to 771 agating modes along each edge for the zigzag geometry, however the 
(a)

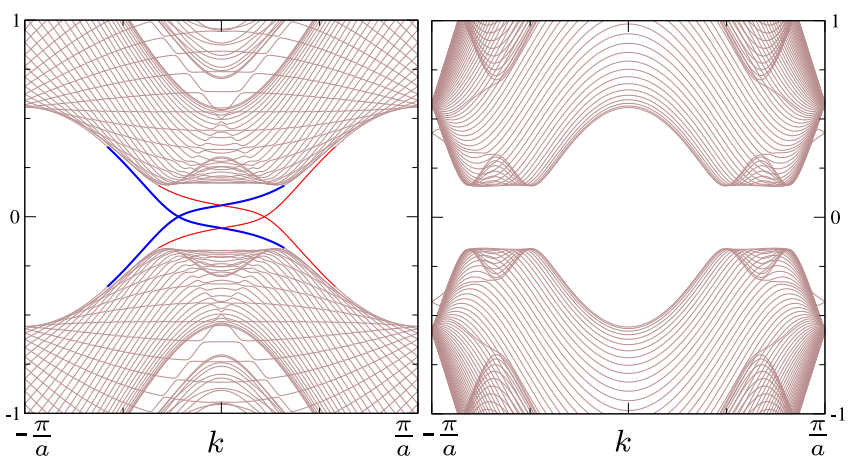

(c)

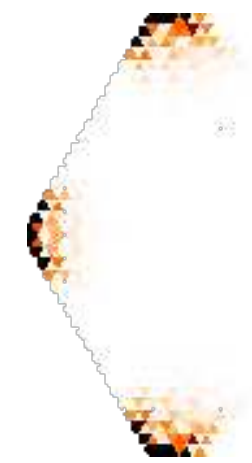

(d)

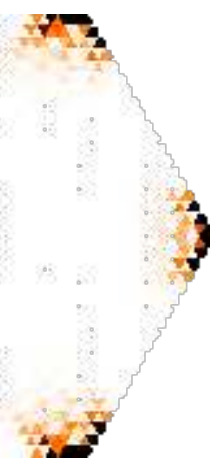

FIG. 5: Exact diagonalisation results for the second-order topological $s_{\tau}$ spin triplet phase. (a), (b) The 1D dispersion of infinite superconducting ribbons with (a) armchair and (b) zigzag terminations. Edge modes propagating along opposite edges are shown in different colors. (c) Wavefunction profile of the six zero energy eigenstates on a flake geometry. These are the subgap states marked in red in the corresponding spectrum displaying 60 eigenstates around zero in (d). Here we use the parameters $\eta=0.2 t, \mu=0.4 t, \Delta^{\prime}=0.033 t$ corresponding to a bulk superconducting gap $\Delta \approx 0.16 t$.

(a)

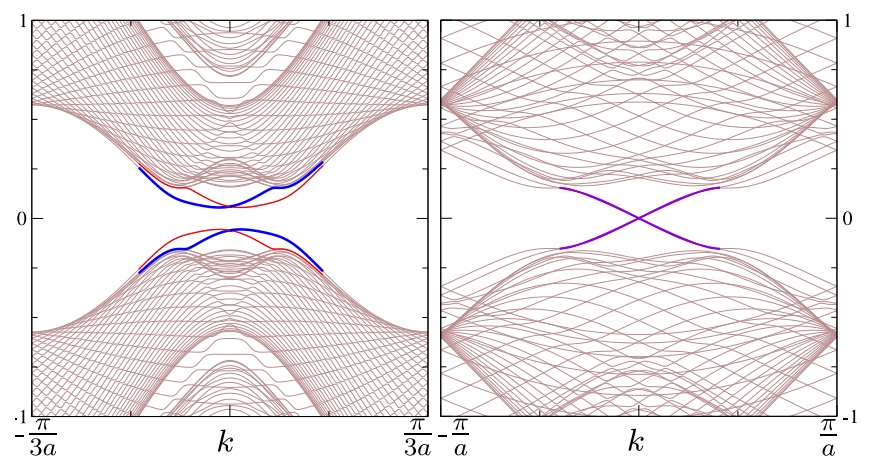

(c)

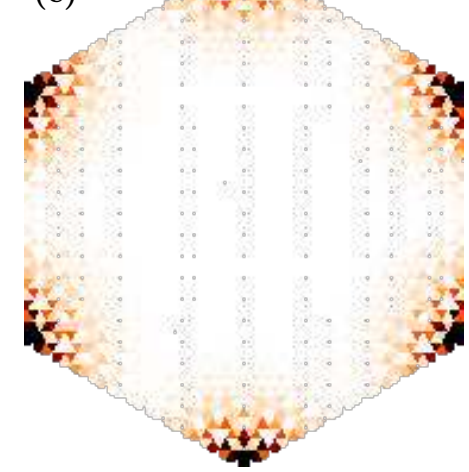

(d)

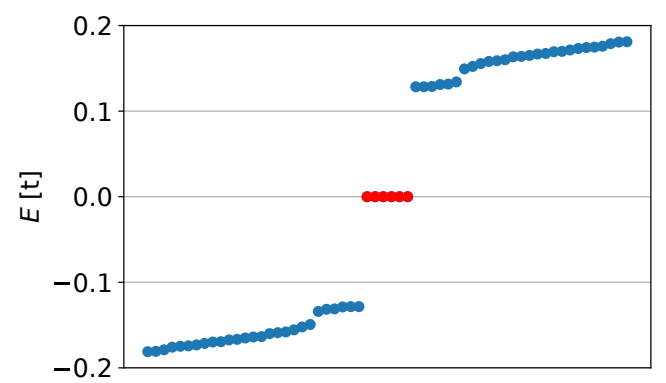

FIG. 6: Exact diagonalisation results for the second-order topological $p+i \tau p$ intravalley spin triplet phase. (a), (b), The 1D dispersion of infinite superconducing ribbons with (a) armchair and (b) zigzag terminations. Edge modes propagating along opposite edges are shown in different colors. (c) Wavefunction profile of the six lowest absolute energy eigenstates on a flake geometry. These are the subgap states marked in red in the corresponding spectrum displaying 60 eigenenergies around zero in (d). Here we use the parameters $\eta=0.2 t$, $\mu=0.4 t, \Delta^{\prime}=0.13 t$, and $\phi=\pi / 2$ corresponding to a bulk superconducting gap $\Delta \approx 0.16 t$.

772 edge behavior for an armchair ribbon is sensitive to the width of the 782 the ribbon width. In Fig. 7 we show the level spectrum at $k=0$ 773 ribbon as well as the value of the pair density wave order parameter 783 as a function of $\phi$ for an armchair ribbon of various widths, (a) $774 \phi$, as shown in Fig. 6. Both the armchair ribbon and flake have ${ }_{784} 35$, (b) 36, (c) 37 unit cells. In all cases, the modes propagating 775 a width of 35 unit cells. In this case, the plotted ribbon disper- 785 along the left and right edges have distinct $1 \mathrm{D}$ dispersions, except at 776 sion with armchair edges is gapped, and the flake exhibits Kramers 786 values $\phi=n \pi / 2$, with $n \in \mathbb{Z}$ for which the gap function $\Delta\left(\boldsymbol{R}, \boldsymbol{R}^{\prime}\right)$ 777 pairs of Majorana corner states on corners between armchair edges 787 is reflection symmetric about the center of the ribbon. We find that 778 at $\phi=\pi / 2$. For $\phi=\pi / 2$, the flake is inversion symmetric and the 788 the edge is always gapped at $\phi=\pi / 2$, when $\Delta\left(\boldsymbol{R}, \boldsymbol{R}^{\prime}\right)$ is odd under 779 zero energy corner modes are a signature of the intrinsic second-order 789 inversion. At $\phi=0$, the gap function is even under inversion and 780 topology of the bulk superconductivity $[1,4]$. 790 no higher-order topology is possible. The edge gap closes for a value 
(a) 0

$\pi / 2$

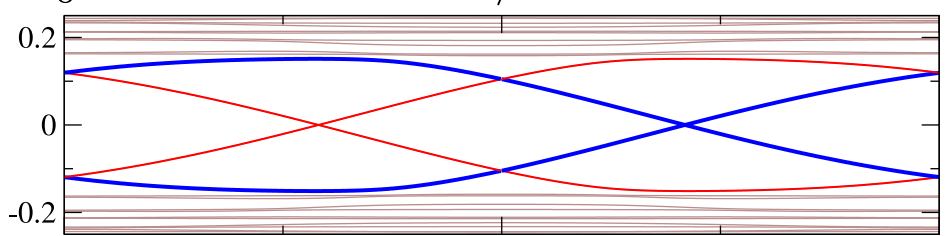

(b)

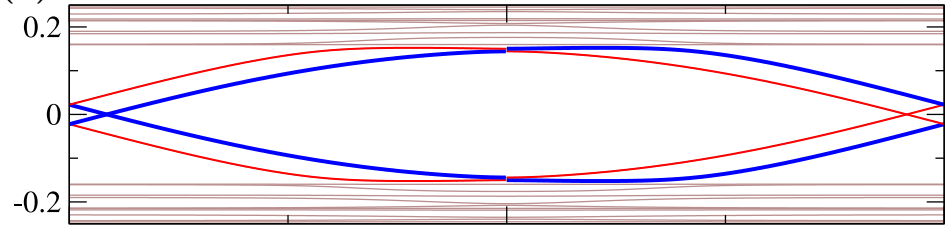

(c)

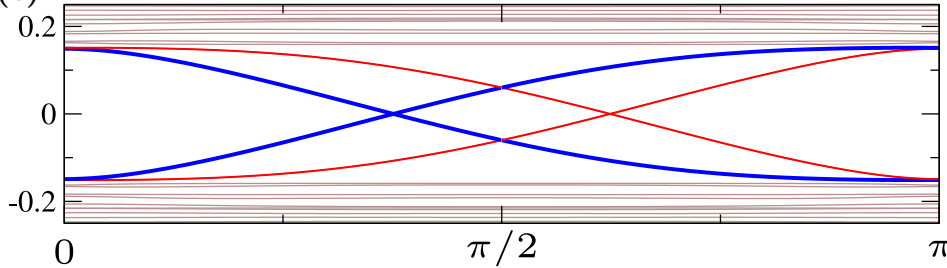

FIG. 7: Dependence of the spectrum (in units of $t$ ) on the pair density wave order parameter $\phi$ in the $p+i \tau p$ state, for ribbons of various width. The energy spectrum at $k=0$ of an infinite superconducting ribbon with armchair termination of width (a) 35, (b) 36 , (c) 37 unit cells as a function of $\phi$, for parameters $\eta=0.2 t, \mu=0.4 t$ and $\Delta^{\prime}=0.13 t$ corresponding to a bulk superconducting gap $\Delta \approx 0.16 t$.

(a)

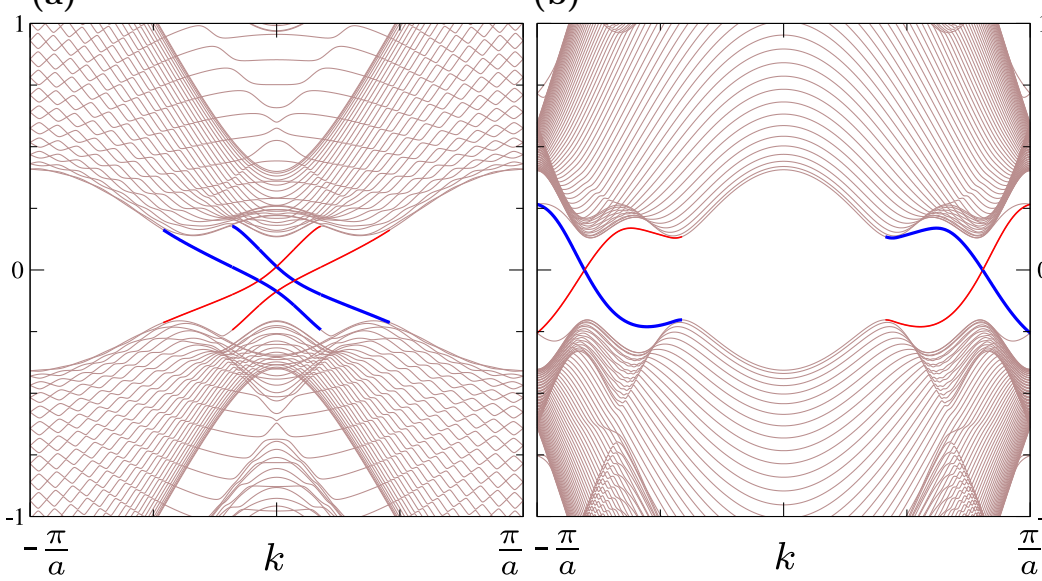

FIG. 8: The 1D dispersion of infinite superconducting ribbons with (a) armchair and (b) zigzag terminations in the $p+i p$ intervalley spin triplet phase, with parameters $\eta=0.2 t, \mu=0.4 t$, and $\Delta^{\prime}=0.2 t$ corresponding to a bulk superconducting gap $\Delta \approx 0.18 t$. Edge modes propagating along opposite edges are shown in different colors.

792 which the Majorana corner modes disappear.

${ }^{793}$ For the intervalley $p+i p$ spin triplet phase, two co-propagating 794 modes are observed on each edge for both the armchair and zigzag 795 geometries, as illustrated in Fig. 8. Keeping in mind that these 796 modes have been obtained from the BdG Hamiltonian in the form 797 of Eq. (40), the chiral edge modes are Dirac fermions, i.e. they are 798 not their own antiparticle.

799

\section{DISCUSSION}

${ }_{800}$ In this paper we considered the phase diagram of an interacting 801 artificial honeycomb superlattice, with Fermi pockets around the ${ }_{802}$ Dirac $K, K^{\prime}$ points, subject to intrinsic spin-orbit coupling, i.e. a 803 doped two-dimensional topological insulator.

${ }_{804}$ We have shown that first and second-order topological supercon${ }_{805}$ ductivity arises purely due to the Coulomb repulsion, an effect which 806 is enhanced in the limit of localised atomic orbitals.

${ }_{807}$ The mechanism has been elucidated for general lattice models with ${ }_{808} C_{6 v}$ point group symmetry, $S U(2)$ spin rotation and time-reversal 809 symmetry in [1], and is extended here in two ways: $(i)$ the influ810 ence of intrinsic spin-orbit coupling is incorporated, breaking spin ${ }_{811} S U(2) \rightarrow U(1)$ generating a $\mathbb{Z}_{2}$ topological bandgap, and (ii) micro${ }_{812}$ scopic modeling for a specific, experimentally promising, material is ${ }_{813}$ presented. However, while our field theory treatment is generic, we ${ }^{814}$ present results specifically for a model of an artificial honeycomb lat815 tice based on a nanopatterned hole-doped semiconductor quantum 816 well, having in mind the fact that in this situation there is a high ${ }_{817}$ degree of experimental control over the electron-electron interaction 818 as well as the band structure.

Our microscopic modeling shows that three distinct (first and 820 second-order) topological superconducting phases emerge for real${ }^{321}$ istic material parameters, and moreover, that these instabilities are 322 the leading weak coupling instabilities of the Fermi surface - with 823 magnetic and charge ordering only setting in at larger interaction $\pi^{-\frac{1}{24}}$ strengths. The superconducting phases are:

825 826 827 828 829 830 831
1. $p+i p$ intervalley, which admits a first-order topological invariant and therefore hosts gapless chiral edge modes; we have shown from numerical calculations that this phase hosts two co-propagating chiral Dirac fermionic edge modes.

2. $p+i \tau p$ intravalley, a spatially modulated pair density wave which hosts two Majorana edge modes of opposite chirality due to the opposite pairing in the two valleys. Hybridization 

846 probed in experiment through STM [22], or through measurements 847 of the Josephson critical current [23]. It has been proposed that 848 higher-order topological superconductors host Majorana states at 849 disinclinations and defects [25-29], a phenomenon currently unex850 plored experimentally, which could offer another signature of higher851 order topology.

852 Very recent progress in $n$-type semiconductors patterned with a 853 honeycomb superlattice [46-48] has clearly demonstrated Dirac band 854 structure features. Our findings show that $p$-type semiconductor 8 855 patterned with a honeycomb superlattice is an enticing avenue to- 882 856 wards topological superconducting phases. The $p$-type semiconduc- 8 857 tor allows for strong intrinsic spin-orbit coupling, which is otherwise 858 negligible in $n$-type. Stronger spin-orbit coupling reduces the effec- 885 — Natural Sciences.
[1] T. Li, M. Geier, J. Ingham and H. D. Scammell, "Higher-order topo- 898 logical superconductivity from repulsive interactions in kagome and 899 honeycomb systems", arXiv:2108.10897 [cond-mat.supr-con]. 900

[2] Y. Peng, Y. Bao, and F. von Oppen, "Boundary Green functions 901 of topological insulators and superconductors", Phys. Rev. B 95, 902 235143 (2017).

[3] J. Langbehn, Y. Peng, L. Trifunovic, F. von Oppen and P. W. 904 Brouwer, "Reflection symmetric second-order topological insulators 905 and superconductors", Phys. Rev. Lett. 119, 246401 (2017).

[4] M. Geier, L. Trifunovic, M. Hoskam and P. W. Brouwer, "Secc order topological insulators and superconductors with an order-two 908 crystalline symmetry", Phys. Rev. B 97, 205135 (2018).
[5] M. Geier, P. W. Brouwer and L. Trifunovic, "Symmetry-based indicators for topological Bogoliubov-de Gennes Hamiltonians", Phys. Rev. B 101, 245128 (2020).

[6] K. Shiozaki, "Variants of the symmetry-based indicator", arXiv:1907.13632 [cond-mat.mes-hall].

[7] S. Ono, H.-C. Po and H. Watanabe, "Refined symmetry indicators for topological superconductors in all space groups", Sci. Adv. 6, eaaz8367 (2020)

[8] L. Trifunovic and P. W. Brouwer, "Higher-order bulk-boundary correspondence for topological crystalline phases", Phys. Rev. X 9 , 011012 (2019). 
[9] L. Trifunovic and P. W. Brouwer, "Higher-order topological band 957 structures", Physica Status Solidi (B), 2020.

[10] E. Khalaf, "Higher-order topological insulators and superconductors 959 protected by inversion symmetry", Phys. Rev. B 97, 205136 (2018). 960

[11] S.-B. Zhang, W. B. Rui, A. Calzona, S.-J. Choi, A. P. Schnyder, 961 B. Trauzettel, "Topological and holonomic quantum computation 962 based on second-order topological superconductors," Phys. Rev. Re- 963 search 2, 043025 (2020).

[12] R.-X. Zhang et al., "higher-order Topology and Nodal Topological 965 Superconductivity in Fe(Se,Te) Heterostructures", Phys. Rev. Lett. 966 123, 167001 (2019).

[13] X. Zhu, "Second-order Topological Superconductors with Mixed 968 Pairing", Phys. Rev. Lett. 122, 236401 (2019).

[14] S. Franca, D. V. Efremov and I. C. Fulga, "Phase tunable second- 970 order topological superconductor", Phys. Rev. B 100, 075415971 (2019).

[15] Z. Wu, Z. Yan and W. Huang, "Higher-order topological supercon- 973 ductivity: possible realization in Fermi gases and $\mathrm{Sr}_{2} \mathrm{RuO}_{4}$ ", Phys. 974 Rev. B 99, 020508 (2019).

[16] B. Roy, "Higher-order topological superconductors in $\mathcal{P}$-, $\mathcal{T}$-odd 976 quadrupolar Dirac materials", Phys. Rev. B 101, 220506 (2020). 977

[17] J. Ahn and B.-J. Yang, "Higher-Order Topological Superconduc- 978 tivity of Spin-Polarized Fermions" Phys. Rev. Research 2, 012060979 (2020).

[18] R.-X. Zhang and S. Das Sarma, "Intrinsic Time-reversal-invariant 981 Topological Superconductivity in Thin Films of Iron-based Super- 982 conductors", Phys. Rev. Lett. 126, 137001 (2021).

[19] A. Chew et al., "Higher-Order Topological Superconductivity in 984 Twisted Bilayer Graphene", arXiv:2108.05373 [cond-mat.supr-con]. 985

[20] Y.-T. Hsu et al., "Inversion-protected higher-order topological su- 986 perconductivity in monolayer WTe 2 ", Phys. Rev. Lett. 125, 097001987 (2020).

[21] Y. Wang, M. Lin and T. L. Hughes, "Weak-pairing higher-order 989 topological superconductors," Phys. Rev. B 98, 165144 (2018). 990

[22] M. J. Gray et al., "Evidence for Helical Hinge Zero Modes in an 991 Fe-Based Superconductor", Nano Lett. 19, 4890 (2019).

[23] Y.-B. Choi et al., "Evidence of higher-order topology in multilayer ${ }_{993}$ $\mathrm{WTe}_{2}$ from Josephson coupling through anisotropic hinge states", 994 Nat. Mater. 19, 974-979 (2020).

[24] F. Schindler et al., "Higher-order topology in bismuth", Nat. Phys. 996 14, 918-924 (2018).

[25] J. C. Y. Teo and T. L. Hughes, "Existence of Majorana-Fermion 998 Bound States on Disclinations and the Classification of Topological 999 Crystalline Superconductors in Two Dimensions", Phys. Rev. Lett. 1000 111, 047006 (2013).

[26] W. A. Benalcazar, J. C. Y. Teo, and T. L. Hughes, "Classification of 1002 two-dimensional topological crystalline superconductors and Majo- 1003 rana bound states at disclinations", Phys. Rev. B 89, 224503 (2014). 1004
[27] X. Zhu, "Tunable Majorana corner states in a two-dimensional second-order topological superconductor induced by magnetic fields", Phys. Rev. B 97, 205134 (2018).

[28] M. Geier, I. C. Fulga and A. Lau, "Bulk-boundary-defect correspondence at disclinations in rotation-symmetric topological insulators and superconductors", SciPost Phys. 10, 092 (2021).

[29] B. Roy and V. Juričić, "Dislocation as a bulk probe of higher-order topological insulators", Phys. Rev. Research 3, 033107 (2021).

[30] T. Li, J. Ingham and H. D. Scammell, "Artificial Graphene: Unconventional Superconductivity in a Honeycomb Superlattice", Phys. Rev. Research 2, 043155 (2020).

[31] M. Polini et al., "Artificial honeycomb lattices for electrons, atoms and photons", Nat. Nano 8, 625-633 (2013).

[32] I. Bloch, J. Dalibard and W. Zwerger, "Many-body physics with ultracold gases", Rev. Mod. Phys. 80, 885 (2008).

[33] D.-W. Zhang et al., "Topological quantum matter with cold atoms", Adv. Phys. 67, 253-402 (2018)

[34] N. R. Cooper, J. Dalibard and I. B. Spielman, "Topological Bands for Ultracold Atoms", Rev. Mod. Phys. 91, 015005 (2019).

[35] A. Browaeys and T. Lahaye, "Many-body physics with individually controlled Rydberg atoms", Nat. Phys.16, 132-142 (2020).

[36] Y. Cao et al., "Unconventional superconductivity in magic-angle graphene superlattices", Nature 556, 43-50 (2018)

[37] Y. Cao et al., "Correlated Insulator Behaviour at Half-Filling in Magic Angle Graphene Superlattices", Nature 556, 80-84 (2018).

[38] H. Yoo et al., "Atomic and electronic reconstruction at the van der Waals interface in twisted bilayer graphene", Nat. Mater. 18, 448 453 (2019).

[39] A. Weston et al., "Atomic reconstruction in twisted bilayers of transition metal dichalcogenides", Nat. Nano 15, 592-597 (2020).

[40] A. K. Geim and I. V. Grigorieva, "Van der Waals heterostructures", Nature 499, 419-425 (2013).

[41] P. Ajayan, P. Kim and K. Banerjee, "Two-dimensional van der Waals materials", Physics Today 69, 9, 38 (2016).

[42] C. Forsythe et al., "Band structure engineering of 2D materials using patterned dielectric superlattices", Nat. Nano. 13, 566-571 (2018).

[43] C.-H. Park and S. G. Louie, "Making Massless Dirac Fermions from Patterned Two-Dimensional Electron Gases", Nano Lett. 9, 17931797 (2009).

[44] M. Gibertini et al., "Engineering artificial graphene in a twodimensional electron gas", Phys. Rev. B 79, 241406(R) (2009).

[45] A. Singha et al., "Two-dimensional Mott-Hubbard electrons in an artificial honeycomb lattice", Science 332, 1176 (2011).

[46] S. Weng et al., "Observation of Dirac bands in artificial graphene in small-period nanopatterned GaAs quantum wells", Nature Nano. 13, 29-33 (2018).

[47] L. Du et al., "Emerging many-body effects in semiconductor artificial graphene with low disorder", Nature Comm. 9, 3299 (2018). 
[48] L. Du et al., "Observation of flat bands in gated semiconductor 1053 artificial graphene", Phys. Rev. Lett. 126, 106402 (2021).

[49] O. A. Tkachenko, V. A. Tkachenko, I. S. Terekhov and O. P. 1055 Sushkov, "Effects of Coulomb screening and disorder on an arti- 1056 ficial graphene based on nanopatterned semiconductor", 2D Mater. 1057 2, 014010 (2015)

[50] T. Li and O. P. Sushkov, "Chern insulating state in laterally pat- 1059 terned semiconductor heterostructures", Phys. Rev. B 94, 1553111060 (2016).

[51] T. Li and O. P. Sushkov, "Two-dimensional topological semimetal 1062 state in a nanopatterned semiconductor system", Phys. Rev. B 96, 1063 085301 (2017).

[52] O. P. Sushkov and A. H. Castro Neto, "Topological Insulating States 1065 in Laterally Patterned Ordinary Semiconductors", Phys. Rev. Lett. 1066 110, 186601 (2013).

[53] H. D. Scammell and O. P. Sushkov, "Tuning the topological insulator 1068 states of artificial graphene", Phys. Rev. B. 99, 085419 (2019). 1069

[54] C. L. Kane and E. J. Mele, "Quantum Spin Hall Effect in Graphene", 1070 Phys. Rev. Lett. 95, 226801 (2005).

[55] L. Wang et al., "Correlated electronic phases in twisted bilayer tran- 1072 sition metal dichalcogenides", Nature Materials 19, 861 (2020). $\quad{ }_{1073}$

[56] F. Wu, T. Lovorn, E. Tutuc, I. Martin and A. H. MacDonald, "Topo- 1074 logical insulators in twisted transition metal dichalcogenide homo- 1075 bilayers", Phys. Rev. Lett. 122, 086402 (2019).

57] A. Devarakonda et al., "Clean 2D superconductivity in a bulk van 1077 der Waals superlattice", Science 370, 231-236 (2020).

[58] X. Yang et al., "Anisotropic superconductivity in topological crys- 1079 talline metal $\mathrm{Pb}_{1 / 3} \mathrm{TaS}_{2}$ with multiple Dirac fermions", Phys. Rev. 1080 B 104, 035157 (2021).

[59] M. Liao et al., "Superconductivity in few-layer stanene" Nat. Phys. 1082 14, 344-348 (2018).

[60] S. C. de la Barrera et al., "Tuning Ising superconductivity with 1084 layer and spin-orbit coupling in two-dimensional transition-metal 1085 dichalcogenides", Nat. Comm. 9, 1427 (2018).

[61] J. Lu et al., "Full superconducting dome of strong Ising protection 1087 in gated monolayer $\mathrm{WS}_{2}$ ", Proc. Natl. Acad. Sci. U.S.A. 115 (14) 1088 3551-3556 (2018).

[62] J. Lu et al., "Evidence for two-dimensional Ising superconductivity 1090 in gated $\mathrm{MoS}_{2}$ ", Science 350, 6266 1353-1357 (2015).

[63] Y. Yang et al., "Enhanced superconductivity upon weakening of 1092 charge density wave transport in $2 \mathrm{H}-\mathrm{TaS}_{2}$ in the two-dimensional 1093 limit", Phys. Rev. B 98, 035203 (2018).

[64] J. T. Ye et al., "Superconducting Dome in a Gate-Tuned Band In- 1095 sulator", Science 338, 1193-1196 (2012).

[65] S. Yonezawa, "Nematic Superconductivity in Doped $\mathrm{Bi}_{2} \mathrm{Se}_{3}$ Topo- 1097 logical Superconductors" Condens. Matter 4(1), 2 (2019).

[66] M. Kreiner et al., "Bulk Superconducting Phase with a Full Energy 1099 Gap in the Doped Topological Insulator $\mathrm{Cu}_{x} \mathrm{Bi}_{2} \mathrm{Se}_{3}$ ", Phys. Rev. ${ }_{1100}$
Lett. 106, 127004 (2011)

[67] S. Sasaki et al., "Odd-Parity Pairing and Topological Superconductivity in a Strongly Spin-Orbit Coupled Semiconductor", Phys. Rev. Lett. 109, 217004 (2012).

[68] Z. Liu et al., "Superconductivity with Topological Surface State in $\mathrm{Sr}_{x} \mathrm{Bi}_{2} \mathrm{Se}_{3} "$, J. Am. Chem. Soc. 137, 10512-105152015, (2015)..

[69] T. Sato et al., "Fermiology of Strongly Spin-Orbit Coupled Superconductor $\mathrm{Sn}_{1-x} \operatorname{In}_{x}$ Te and its Implication to Topological Superconductivity", Phys. Rev. Lett. 110, 206804 (2013).

[70] M. Novak et al., "Unusual nature of fully-gapped superconductivity in In-doped SnTe", Phys. Rev. B 88, 140502 (2013).

[71] L. Fu and E. Berg, "Odd-Parity Topological Superconductors: Theory and Application to $\mathrm{Cu}_{x} \mathrm{Bi}_{2} \mathrm{Se}_{3}$ ", Phys. Rev. Lett. 105, 097001 (2010).

[72] V. Fatemi et al., "Electrically tunable low-density superconductivity in a monolayer topological insulator", Science 3626417 926-929 (2018).

[73] E. Sajadi et al., "Gate-induced superconductivity in a monolayer topological insulator", Science 3626417 922-925 (2018).

[74] B. R. Ortiz et al., " $\mathrm{CsV}_{3} \mathrm{Sb}_{5}: \mathrm{A} \mathbb{Z}_{2}$ Topological Kagome Metal with a Superconducting Ground State", Phys. Rev. Lett. 125, 247002 (2020).

[75] C. C. Zhu et al., "Double-dome superconductivity under pressure in the V-based Kagome metals $\mathrm{AV}_{3} \mathrm{Sb}_{5}(\mathrm{~A}=\mathrm{Rb}$ and $\mathrm{K})$ ", https://arxiv.org/abs/2104.14487.

[76] K. Y. Chen et al., "Double Superconducting Dome and Triple Enhancement of $\mathrm{T}_{c}$ in the Kagome Superconductor $\mathrm{CsV}_{3} \mathrm{Sb}_{5}$ under High Pressure", Phys. Rev. Lett. 126, 247001 (2021).

[77] B. R. Ortiz et al., "Superconductivity in the $\mathbb{Z}_{2}$ kagome metal $\mathrm{KV}_{3} \mathrm{Sb}_{5}$ ", Phys. Rev. Materials 5, 034801 (2021).

[78] S. Ni et al., "Anisotropic superconducting properties of Kagome metal $\mathrm{CsV}_{3} \mathrm{Sb}_{5} "$, Chin. Phys. Lett. 38057403 (2021).

[79] H. Chen et al., "Roton pair density wave and unconventional strongcoupling superconductivity in a topological kagome metal", Phys. Rev. X 11, 031026 (2021).

[80] Z. Liang et al., "Three-dimensional charge density wave and robust zero-bias conductance peak inside the superconducting vortex core of a kagome superconductor $\mathrm{CsV}_{3} \mathrm{Sb}_{5}$ "Phys. Rev. X 11, 031026 (2021).

[81] J. Zhao, "Electronic correlations in the normal state of the kagome superconductor KV $\mathrm{Kb}_{5}$ ", Phys. Rev. B 103, L241117 (2021).

[82] M. Kang et al., "Twofold van Hove singularity and origin of charge order in topological kagome superconductor $\mathrm{CsV}_{3} \mathrm{Sb}_{5}$ ", arXiv:2105.01689 [cond-mat.str-el].

[83] Y.-X. Jiang et al., "Unconventional chiral charge order in kagome superconductor $\mathrm{KV}_{3} \mathrm{Sb}_{5}$ ", Nat. Mater. (2021).

[84] H. Li et al., "Rotation symmetry breaking in the normal state of a kagome superconductor $\mathrm{KV}_{3} \mathrm{Sb}_{5}$ ", arXiv:2104.08209 [condmat.supr-con]. 
[85] B. R. Ortiz et al., "New kagome prototype materials: discovery 1135 of $\mathrm{KV}_{3} \mathrm{Sb}_{5}, \mathrm{RbV}_{3} \mathrm{Sb}_{5}$, and $\mathrm{CsV}_{3} \mathrm{Sb}_{5}$ " Phys. Rev. Mat. 3, 0944071136 (2019).

[86] H. Zhao et al., "Cascade of correlated electron states in a kagome ${ }_{1138}$ superconductor $\mathrm{CsV}_{3} \mathrm{Sb}_{5}$ ” arXiv:2103.03118 [cond-mat.supr-con]. ${ }_{1139}$

[87] H. X. Li et al., "Observation of Unconventional Charge Density 1140 Wave without Acoustic Phonon Anomaly in Kagome Superconduc- 1141 tors $\mathrm{AV}_{3} \mathrm{Sb}_{5}$ (A=Rb,Cs)". arXiv:2103.09769 (2021).

[88] T. Qian et al., "Revealing the competition between charge-density 1143 wave and superconductivity in $\mathrm{CsV}_{3} \mathrm{Sb}_{5}$ through uniaxial strain" ${ }_{1144}$ arXiv:2107.04545 (2021)

89] M. H. Christensen et al., "Theory of the charge-density wave in 1146 $A \mathrm{~V}_{3} \mathrm{Sb}_{5}$ kagome metals" arXiv:2107.04546 [cond-mat.supr-con]. ${ }_{1147}$

[90] H. Tan et al., "Charge density waves and electronic properties of 1148 superconducting kagome metals", Phys. Rev. Lett. 127, 0464011149 (2021).

1150

[91] T. Park, M. Ye and L. Balents "Electronic instabilities of kagome 1151 metals: saddle points and Landau theory", Phys. Rev. B 104, 1152 [10 035142 (2021).

1153

[92] X. Wu et al., "Nature of unconventional pairing in the kagome su- 1154 perconductors $\mathrm{AV}_{3} \mathrm{Sb}_{5}$ ", arXiv:2104.05671 [cond-mat.supr-con]. 1155

[93] Y.-P. Lin and R. M. Nandkishore, "Kagome superconductors 1156 from Pomeranchuk fluctuations in charge density wave metals", 1157 arXiv:2107.09050 [cond-mat.str-el].

1158

94] K. Seo, B. A. Bernevig and J. Hu, "Pairing Symmetry in a Two- 1159 Orbital Exchange Coupling Model of Oxypnictides", Phys. Rev. 1160 Lett. 101, 206404 (2008).

[95] T. Hanaguri, S. Niitaka, K. Kuroki and H. Takagi, "Unconventional 1162 s-Wave Superconductivity in Fe(Se,Te)" Science 328 474-476 (2010). 1163

[96] P. J. Hirschfeld, M. M. Korshunov, and I. I. Mazin, "Gap symmetry 1164 and structure of Fe-based superconductors", Rep. Prog. Phys. 741165 124508 (2011).

[97] Y. Bang and G. R. Stewart, "Superconducting properties of the $\mathrm{s}_{ \pm}-$ wave state: Fe-based superconductors", J. Phys.: Condens. Matter
29123003 (2017).

[98] R.-X. Zhang, W. S. Cole and S. Das Sarma, "Helical Hinge Majorana Modes in Iron-Based Superconductors", Phys. Rev. Lett. 122, 187001 (2019)

[99] S. Qin, C. Fang, F.-C. Zhang and J. Hu, "Topological Superconductivity in an $s$-wave Superconductor and Its Implication to Iron-based Superconductors", arXiv:2106.04200 [cond-mat.supr-con].

[100] R. Winkler, Spin-Orbit Coupling Effects in Two-Dimensional Electron and Hole Systems (Springer-Verlag, Berlin, 2003).

101] V. N. Kotov, B. Uchoa and A. H. Castro Neto, " $1 / N$ expansion in correlated graphene", Phys. Rev. B 80, 165424 (2009).

102] X.F. Wang and T. Chakraborty, "Collective excitations of Dirac electrons in a graphene layer with spin-orbit interactions", Phys. Rev. B 75, 033408 (2007).

[103] A. Thakur, R. Sachdeva and A. Agarwal, "Dynamical polarizability, screening, and plasmons in one, two, and three dimensional massive Dirac systems", J. Phys.: Condens. Matter 29105701 (2016).

104] P.K. Pyatkovskiy "Dynamical polarization, screening, and plasmons in gapped graphene",J. Phys.: Condens. Matter 21, 025506 (2009).

$5]$ M. Sigrist, "Introduction to Unconventional Superconductivity", AIP Conference Proceedings 789, 165 (2005).

106] R. Samajdar and M. S. Scheurer, "Microscopic pairing mechanism, order parameter, and disorder sensitivity in moiré superlattices: Applications to twisted double-bilayer graphene", Phys. Rev. B 102, 064501 (2020)

107] A. Altland and M. Zirnbauer, "Nonstandard symmetry classes in mesoscopic normal-superconducting hybrid structures", Phys. Rev. B 55, 1142 (1997).

108] A. P. Schnyder, S. Ryu, A. Furusaki, and A. W. W. Ludwig, "Classification of topological insulators and superconductors in three spatial dimensions", Phys. Rev. B 78, 195125 (2008). 


\section{Appendices}

\section{Appendix A: Deriving the Effective Hamiltonian}

\section{Superlattice potential}

For a superlattice placed on top of the 2DHG heterostructure, the superlattice potential has a $z$ dependence,

$$
W(\boldsymbol{r}, z)=2 W_{0} \sum_{i} \cos \left(\boldsymbol{G}_{i} \cdot \boldsymbol{r}\right) e^{-\left(z+z_{0}\right) G_{0}},
$$

where $\boldsymbol{G}_{i}$ are the reciprocal lattice vectors connecting corners of the hexagonal Brillouin zone Figure 1 . Here, $z=0$ is the center of the ${ }_{1171}$ quantum well and $z_{0}$ is the distance from the superlattice to the center of the quantum well. This top-gate superlattice breaks inversion ${ }_{1172}$ symmetry, but one may argue that parity breaking effects are exponentially suppressed and may be ignored in the regime where $z_{0} G \ll 1$. ${ }_{1173}$ Alternatively, we can envisage placing a superlattice on both the top and bottom gates - preserving parity. This is captured by,

$$
W(\boldsymbol{r}, z)=2 W \sum_{i} \cos \left(\boldsymbol{G}_{i} \cdot \boldsymbol{r}\right) e^{-z_{0} G_{0}} \cosh \left(z G_{0}\right) .
$$

\section{Effective Dirac Hamiltonian}

We explicitly obtain the effective Dirac Hamiltonian via,

$$
\left(\mathcal{H}_{0}\right)_{s, \tau, \sigma ; s^{\prime}, \tau^{\prime}, \sigma^{\prime}}=\left\langle s, \tau, \sigma, \boldsymbol{p}\left|\mathcal{H}_{2 D H G+W}\right| s^{\prime}, \tau^{\prime}, \sigma^{\prime}, \boldsymbol{p}\right\rangle
$$

where the wavefunctions $|s, \tau, \sigma, \boldsymbol{p}\rangle$ are obtained as an expansion in $\boldsymbol{p}$ near the Dirac points $\boldsymbol{K}_{i}$. Explicitly, they are

$$
\langle\boldsymbol{r} \mid s, \tau, \sigma, \boldsymbol{p}\rangle=\sum_{j=1}^{3} \bar{A}_{j, s, \tau} C_{\sigma, j} e^{i \tau \boldsymbol{K}_{j} \cdot \boldsymbol{r}} e^{i \boldsymbol{p} \cdot \boldsymbol{r}}
$$

where

$$
\bar{A}_{j, s=\uparrow, \tau}=\left(\begin{array}{c}
a_{3 / 2}\left(K_{0}\right) \hat{o} \\
i a_{1 / 2}\left(K_{0}\right) \tau e^{i 2 \pi(j-1) / 3} \hat{e} \\
a_{-1 / 2}\left(K_{0}\right) e^{i 4 \pi(j-1) / 3} \hat{o} \\
-i a_{-3 / 2}\left(K_{0}\right) \tau e^{i 2 \pi(j-1)} \hat{e}
\end{array}\right), \bar{A}_{j, s=\downarrow, \tau}=\left(\begin{array}{c}
i a_{-3 / 2}\left(K_{0}\right) \tau e^{-2 \pi(j-1)} \hat{e} \\
a_{-1 / 2}\left(K_{0}\right) e^{-i 4 \pi(j-1) / 3} \hat{o} \\
-i a_{1 / 2}\left(K_{0}\right) \tau e^{-i 2 \pi(j-1) / 3} \hat{e} \\
a_{3 / 2}\left(K_{0}\right) \hat{o}
\end{array}\right), C_{\sigma, j}=\frac{1}{\sqrt{3}} e^{i 2 \pi(j-1) \sigma / 3}
$$

${ }_{1179}$ and $\bar{A}_{j, s=\downarrow, \tau}=\mathcal{U} \mathcal{K} \bar{A}_{j, s=\uparrow,-\tau}$. Here, $\mathcal{K}$ is complex conjugation and $\mathcal{U}=i e^{i \pi S_{y}}$ with $S_{x}$ in the spin-3/2 representation. The complex phase 1180 is given by the in-plane momenta $e^{i \phi}=\left(p_{x}+i p_{y}\right) / p$, with $p=|\boldsymbol{p}|$ and the coefficients $a_{S_{z}}(p)$ are found numerically via exact diagonalisation 1181 of the Luttinger Hamiltonian (1), shown in Fig. 9. Finally, we have introduced two orthogonal vectors $\hat{e}, \hat{o}$, which account for the even and 1182 odd parity (inversion in $z$-axis) of the wave function/spin components, $a_{S_{z}}(p, z)$.

${ }_{1183}$ For completeness we also specify the symmetry properties of the wavefunctions. The symmetries of a honeycomb system are $2 \pi / 3$ and $\pi$ 1184 rotations, reflections, and time reversal. At the high symmetry points $\boldsymbol{p}=\mathbf{0}$, using the explicit form of the wavefunctions (A4) and (A5) 

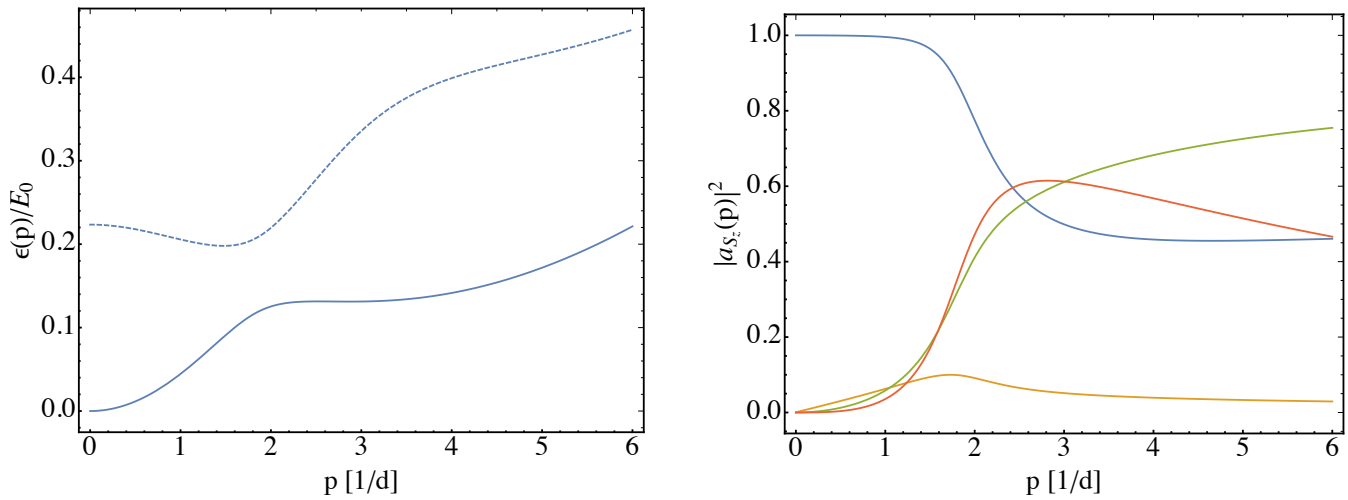

FIG. 9: (a) 2DHG spectrum, $\epsilon(p) / E_{0}$ for InAs. Solid blue line corresponds to the doubly degenerate spectrum that enters the computations of the effective Dirac Hamiltonian (4), the dashed line is the next highest subband, which is ignored in our approximations. (b) Probability densities $\left|a_{S_{z}}(p)\right|^{2}$ of each physical spin component $S_{z}$, presented in (A5).

1185 the transformations are found to be

$$
\begin{aligned}
D\left(C_{3 z}\right)|s, \tau, \sigma\rangle & =-e^{2 i \pi \sigma / 3}|s, \tau, \sigma\rangle \\
D\left(C_{2 z}\right)|s, \tau, \sigma\rangle & =-i s|s,-\tau, \sigma\rangle \\
D\left(C_{2 x}\right)|s, \tau, \sigma\rangle & =i|-s, \tau,-\sigma\rangle \\
D\left(C_{2 y}\right)|s, \tau, \sigma\rangle & =-s|-s,-\tau,-\sigma\rangle \\
D(\mathcal{T})|s, \tau, \sigma\rangle & =-s|-s,-\tau,-\sigma\rangle .
\end{aligned}
$$

The resulting transformation properties are summarized in Table II. 


\section{Preliminaries}

The polarisation operators are given by (setting velocity $v=1$ for ease of notation)

$$
i \Pi_{\mu \nu}\left(p_{0}, \boldsymbol{p}\right)=\int \frac{d q_{0} d^{2} q}{(2 \pi)^{3}} \frac{\operatorname{Tr}\left[J^{\mu} h^{\alpha} J^{\nu} h^{\beta}\right]}{\tilde{q}_{0}^{2}-\boldsymbol{q}^{2}-\eta^{2}} \frac{\tilde{q}_{\beta}(\tilde{q}+\tilde{p})_{\alpha}}{\left(\tilde{q}_{0}+p_{0}\right)^{2}-(\boldsymbol{q}+\boldsymbol{p})^{2}-\eta^{2}}
$$

with modified momenta $\tilde{q}_{\alpha} \in\left\{q_{0}+\mu, \boldsymbol{q}, \eta\right\}, \tilde{p}_{\alpha} \in\left\{p_{0}+\mu, \boldsymbol{q}, 0\right\}$, and vertices: $h^{\alpha} \in\left\{\mathbb{1}, \sigma_{x}, \tau_{z} \sigma_{y}, s_{z} \tau_{z} \sigma_{z}\right\}$, pertaining to the Hamiltonian, 1191 and $J_{I}^{\mu} \in\left\{\mathbb{1}, \sigma_{ \pm}, \tau_{z} \sigma_{z}, \tau_{z} s_{z}, \tau_{z} s_{z} \sigma_{ \pm}, \sigma_{z} s_{z}\right\}, J_{I I}^{\mu} \in\left\{\mathbb{1}, \sigma_{ \pm}, \sigma_{z} s_{z}\right\} \otimes \tau_{ \pm}$, pertaining to the interactions.

${ }_{1192}$ By evaluating the frequency integral by residues, the expression reduces to

$$
\begin{aligned}
i \Pi_{\mu \nu}(p) & =\int \frac{d^{2} q}{(2 \pi)^{3}} \frac{\operatorname{Tr}\left[J^{\mu} h^{\alpha} J^{\nu} h^{\beta}\right]}{q_{0}^{2}-\boldsymbol{q}^{2}-\eta^{2}} \frac{q_{\beta}(q+p)_{\alpha}}{\left(q_{0}+p_{0}\right)^{2}-(\boldsymbol{q}+\boldsymbol{p})^{2}-\eta^{2}}\left[1-\left(\Theta\left(\mu-\varepsilon_{q}\right)+\Theta\left(\mu-\varepsilon_{\boldsymbol{q}+\boldsymbol{p}}\right)\right)\right] \\
& \equiv i \Pi_{\mu \nu}^{(0)}(p)+i \delta \Pi_{\mu \nu}(p)
\end{aligned}
$$

where $\Pi_{\mu \nu}^{(0)}(p)$ is defined as the polarization operator at zero chemical potential $\mu=0$, i.e. the interband polarization operator [30]. The

remaining contribution $\delta \Pi_{\mu \nu}(p)$ is referred to as the intraband polarization operator.

$$
i \Pi_{\mu \nu}^{(0)}(p)=\int_{0}^{1} d y \int \frac{d^{3} l}{(2 \pi)^{3}} \frac{\operatorname{Tr}\left[J^{\mu} h^{\alpha} J^{\nu} h^{\beta}\right](l-y p)_{\beta}(l+(1-y) p)_{\alpha}}{\left[l^{2}-\Delta\left(p_{0}, \boldsymbol{p}, y\right)\right]^{2}}
$$

1196 with $\Delta\left(p_{0}, \boldsymbol{p}, y\right)=\eta^{2}-y(1-y)\left(p_{0}^{2}-\boldsymbol{p}^{2}\right)$. The expression (B2) is evaluated analytically, at zero frequency $p_{0}=0$, and for all $\mu, \nu$; the ${ }_{1197}$ results are printed in Appendix B 2. The second contribution $\delta \Pi_{\mu \nu}(p)$, which depends on chemical potential $\mu$, can be evaluated through 1198 the rearrangements,

$$
\begin{aligned}
\delta \Pi_{\mu \nu}(p) & =\int \frac{d^{2} q}{(2 \pi)^{2}} \frac{\operatorname{Tr}\left[J^{\mu} h^{\alpha} J^{\nu} h^{\beta}\right] q_{\beta}(q+p)_{\alpha} \Theta\left(\mu-\varepsilon_{q}\right)}{2 \varepsilon_{q}\left[\left(\varepsilon_{q}+p_{0}\right)^{2}-(\boldsymbol{q}+\boldsymbol{p})^{2}-\eta^{2}\right]}+\frac{\operatorname{Tr}\left[J^{\mu} h^{\alpha} J^{\nu} h^{\beta}\right] q_{\beta}(q+p)_{\alpha} \Theta\left(\mu-\varepsilon_{\boldsymbol{q}+\boldsymbol{p}}\right)}{2 \varepsilon_{\boldsymbol{q}+\boldsymbol{p}}\left[\left(\varepsilon_{\boldsymbol{q}+\boldsymbol{p}}-p_{0}\right)^{2}-q^{2}-\eta^{2}\right]} \\
& =-\sum_{\sigma= \pm} \sigma \int \frac{d q d \theta}{(2 \pi)^{2}} \frac{\operatorname{Tr}\left[J^{\mu} h^{\alpha} J^{\nu} h^{\beta}\right] q_{\beta}(q+\sigma p)_{\alpha}}{\cos \theta+a_{\sigma}} \frac{\Theta\left(\mu-\varepsilon_{q}\right)}{4 \varepsilon_{q} p}
\end{aligned}
$$

where,

$$
a_{\sigma}=\frac{q^{2}+p^{2}+\eta^{2}-\left(\varepsilon_{q}+\sigma p_{0} e^{i 0}\right)^{2}}{2 q p \sigma}, a_{\sigma}^{0} \equiv a_{\sigma}\left(p_{0}=0\right)=\sigma \frac{p}{2 q}
$$

We specialise to the static limit $p_{0}=0$, which means we only need to keep the principle value of $1 /\left(a_{\sigma}+\cos \theta\right)$

\section{Results}

We decomposed the polarization operator into interband and intraband contributions,

$$
\Pi_{\mu s \tau}\left(p_{0}, \boldsymbol{p}\right)=\Pi_{\mu s \tau}^{0}\left(p_{0}, \boldsymbol{p}\right)+\delta \Pi_{\mu s \tau}\left(p_{0}, \boldsymbol{p}\right) .
$$

indices $\mu=0, x, y, z, s=0, s_{z}, \tau=0, \tau_{z}$. We denote the relatively few distinct non-zero polarization operators as

$$
\begin{aligned}
\Pi_{ \pm}(\boldsymbol{p}) & =\Pi_{z 00, x 00}(0, \boldsymbol{p}) \pm i \Pi_{z 00, y 00}(0, \boldsymbol{p})=\Pi_{z s \tau, x s \tau}(0, \boldsymbol{p}) \pm i \Pi_{z s \tau, y s \tau}(0, \boldsymbol{p}), \\
\Pi_{0}(\boldsymbol{p}) & =\Pi_{0 s \tau, 0 s \tau}(0, \boldsymbol{p}), \\
\Pi_{z} & =\Pi_{z s \tau, z s \tau}(0, \boldsymbol{p}), \\
\Pi_{\eta} & =\Pi_{z s_{z} 0 ; 0 s_{z} \tau}(\boldsymbol{p})=\Pi_{z 0 \tau_{z} ; 000}(\boldsymbol{p})
\end{aligned}
$$


${ }_{1204}$ Here we factor out $N=8$ coming from the trace (spin $\times$ pseudospin $\times$ valley). Subscripts $x, y, z$ correspond to pseudospin, while $s, \tau$ ${ }_{1205}$ correspond to spin $s_{z}$ and valley $\tau_{z}$. We then calculate the following expressions,

$$
\begin{aligned}
i \Pi_{0 s ; 0 s}^{(0)}(\boldsymbol{p}) & =N \int_{0}^{1} d y \int \frac{d^{d} l}{(2 \pi)^{d}} \frac{l_{0}^{2}+l_{x}^{2}+l_{y}^{2}-y(1-y) \boldsymbol{p}^{2}+\eta^{2}}{\left[l^{2}-\Delta\left(p_{0}, \boldsymbol{p}, y\right)\right]^{2}} \\
& =N \int_{0}^{1} d y\left[\frac{1}{2} \frac{i \Gamma(1-d / 2)}{(4 \pi)^{d / 2} \Gamma(2)} \Delta^{d / 2-1}+\left(\eta^{2}-y(1-y) \boldsymbol{p}^{2}\right) \frac{i \Gamma(2-d / 2)}{(4 \pi)^{d / 2} \Gamma(2)} \Delta^{d / 2-2}\right] \\
& =N \frac{i}{8 \pi} \int_{0}^{1} d y\left[-\left(\eta^{2}+y(1-y) \boldsymbol{p}^{2}\right)^{1 / 2}+\left(\eta^{2}-y(1-y) \boldsymbol{p}^{2}\right)\left(\eta^{2}+y(1-y) \boldsymbol{p}^{2}\right)^{-1 / 2}\right] \\
& =N \frac{i}{8 \pi} \int_{0}^{1} d y\left[-2 y(1-y) \boldsymbol{p}^{2}\left(\eta^{2}+y(1-y) \boldsymbol{p}^{2}\right)^{-1 / 2}\right] \\
& =N \frac{i}{8 \pi}\left[-\eta+\frac{1}{2 p}\left(4 \eta^{2}-p^{2}\right) \arcsin \left[\frac{p}{\sqrt{4 \eta^{2}+p^{2}}}\right]\right]
\end{aligned}
$$

$$
\begin{aligned}
i \Pi_{z s ; z s}^{(0)}(\boldsymbol{p}) & =N \frac{i}{8 \pi}\left[2 \eta+\frac{1}{p}\left(4 \eta^{2}+p^{2}\right) \arcsin \left[\frac{p}{\sqrt{4 \eta^{2}+p^{2}}}\right]\right] \\
i \Pi_{x s ; x s}^{(0)}(\boldsymbol{p}) & =N \frac{i}{8 \pi} \frac{p_{y}^{2}}{p^{2}}\left[\eta-\frac{1}{2 p}\left(4 \eta^{2}-p^{2}\right) \arcsin \left[\frac{p}{\sqrt{4 \eta^{2}+p^{2}}}\right]\right], \\
i \Pi_{y s ; y s}^{(0)}(\boldsymbol{p})= & N \frac{i}{8 \pi} \frac{p_{x}^{2}}{p^{2}}\left[\eta-\frac{1}{2 p}\left(4 \eta^{2}-p^{2}\right) \arcsin \left[\frac{p}{\sqrt{4 \eta^{2}+p^{2}}}\right]\right], \\
i \Pi_{x s ; y s}^{(0)}(\boldsymbol{p})= & i \Pi_{y s ; x s}^{(0)}(\boldsymbol{p})=N \frac{i}{8 \pi} \frac{p_{x} p_{y}}{p^{2}}\left[-\eta+\frac{1}{2 p}\left(4 \eta^{2}-p^{2}\right) \arcsin \left[\frac{p}{\sqrt{4 \eta^{2}+p^{2}}}\right]\right], \\
i \Pi_{x 00 ; 0 s_{z} \tau_{z}}^{(0)}(\boldsymbol{p})= & -i \Pi_{0 s_{z} \tau_{z} ; x 00}^{(0)}(\boldsymbol{p})=i \Pi_{x s \tau ; 000}^{(0)}(\boldsymbol{p})=-i \Pi_{000 ; x s \tau}^{(0)}(\boldsymbol{p}) \\
= & -\frac{i N}{8 \pi} \frac{i p_{y}}{p}\left[2 \eta \arcsin \left[\frac{p}{\sqrt{4 \eta^{2}+p^{2}}}\right]\right] \\
i \Pi_{y 00 ; 0 s_{z} \tau_{z}}^{(0)}(\boldsymbol{p})= & -i \Pi_{0 s_{z} \tau_{z} ; y \bar{s}}^{(0)}(\boldsymbol{p})=i \Pi_{y s \tau ; 000}^{(0)}(\boldsymbol{p})=-i \Pi_{000 ; y s \tau}^{(0)}(\boldsymbol{p}) \\
& =\frac{i N}{8 \pi} \frac{i p_{x}}{p}\left[2 \eta \arcsin \left[\frac{p}{\sqrt{4 \eta^{2}+p^{2}}}\right]\right]
\end{aligned}
$$


We now consider the contribution explicitly dependent upon the chemical potential. Again with $p_{0}=0$, one finds

$$
\begin{aligned}
& \delta \Pi_{0 s ; 0 s}(\boldsymbol{p})=\frac{N}{8 \pi}\left[-2 \mu+\eta-\frac{1}{2 p}\left(4 \eta^{2}-p^{2}\right) \arcsin \left(\frac{p}{\sqrt{4 \eta^{2}+p^{2}}}\right)\right] \\
& \delta \Pi_{x ; x}(\boldsymbol{p})=\frac{N}{8 \pi} \frac{p_{y}^{2}}{p^{2}}\left[-\eta+\frac{1}{2 p}\left(4 \eta^{2}-p^{2}\right) \arcsin \left(\frac{p}{\sqrt{4 \eta^{2}+p^{2}}}\right)\right] \\
& \delta \Pi_{y ; y}(\boldsymbol{p})=\frac{N}{8 \pi} \frac{p_{x}^{2}}{p^{2}}\left[-\eta+\frac{1}{2 p}\left(4 \eta^{2}-p^{2}\right) \arcsin \left(\frac{p}{\sqrt{4 \eta^{2}+p^{2}}}\right)\right] \\
& \delta \Pi_{z ; z}(\boldsymbol{p})=\frac{N}{8 \pi}\left[2 \mu-2 \eta-\frac{1}{p}\left(4 \eta^{2}+p^{2}\right) \arcsin \left(\frac{p}{\sqrt{4 \eta^{2}+p^{2}}}\right)\right] \\
& \delta \Pi_{x ; y}(\boldsymbol{p})=\frac{N}{8 \pi} \frac{p_{x} p_{y}}{p^{2}}\left[\eta-\frac{1}{2 p}\left(4 \eta^{2}-p^{2}\right) \arcsin \left(\frac{p}{\sqrt{4 \eta^{2}+p^{2}}}\right)\right] \\
& \delta \Pi_{x ; z}(\boldsymbol{p})=-\frac{N}{8 \pi} i p_{y} \\
& \delta \Pi_{y ; z}(\boldsymbol{p})=\frac{N}{8 \pi} i p_{x} \\
& \delta \Pi_{x 00 ; 0 \tau}(\boldsymbol{p})=\frac{N}{8 \pi} \frac{i 2 \eta p_{y}}{p} \arcsin \left(\frac{\sqrt{p^{2}}}{\sqrt{4 \eta^{2}+p^{2}}}\right) \\
& \delta \Pi_{x s \tau ; 000}(\boldsymbol{p})=\frac{N}{8 \pi} \frac{i 2 \eta p_{y}}{p} \arcsin \left(\frac{\sqrt{p^{2}}}{\sqrt{4 \eta^{2}+p^{2}}}\right) \\
& \delta \Pi_{y 00 ; 0 s \tau}(\boldsymbol{p})=-\frac{N}{8 \pi} \frac{i 2 \eta p_{x}}{p} \arcsin \left(\frac{\sqrt{p^{2}}}{\sqrt{4 \eta^{2}+p^{2}}}\right) \\
& \delta \Pi_{y s \tau ; 000}(\boldsymbol{p})=-\frac{N}{8 \pi} \frac{i 2 \eta p_{x}}{p} \arcsin \left(\frac{\sqrt{p^{2}}}{\sqrt{4 \eta^{2}+p^{2}}}\right) \\
& \delta \Pi_{z s_{z} 0 ; 0 s \tau}(\boldsymbol{p})=\delta \Pi_{z 0 \tau_{z} ; 000}(\boldsymbol{p})=\frac{N}{8 \pi} 2 \eta
\end{aligned}
$$

Now (with $s=0, s_{z}$ ), we relate to channel $I$ (here ordering of $\tau \bar{\tau}$ does not affect the result):

$$
\begin{aligned}
\Pi_{0 ; 0}^{\tau \bar{\tau}}(\boldsymbol{p}) & =\frac{1}{2} \Pi_{z ; z}(\boldsymbol{p}) \\
\Pi_{x ; x}^{\tau \bar{\tau}}(\boldsymbol{p}) & =\frac{1}{2} \Pi_{y ; y}(\boldsymbol{p}) \\
\Pi_{y ; y}^{\tau \bar{\tau}}(\boldsymbol{p}) & =\frac{1}{2} \Pi_{x ; x}(\boldsymbol{p}) \\
\Pi_{z ; z}^{\tau \bar{\tau}}(\boldsymbol{p}) & =\frac{1}{2} \Pi_{0 ; 0}(\boldsymbol{p}) \\
\Pi_{x ; y}^{\tau \bar{\tau}}(\boldsymbol{p}) & =-\frac{1}{2} \Pi_{x ; y}(\boldsymbol{p}) \\
\Pi_{x ; 0}^{\tau \bar{\tau}}(\boldsymbol{p}) & =0 \\
\Pi_{y ; 0}^{\tau \bar{\tau}}(\boldsymbol{p}) & =0 \\
\Pi_{z ; 0}^{\tau \bar{\tau}}(\boldsymbol{p}) & =\frac{1}{2} \Pi_{z s ; 0}(\boldsymbol{p})=N \frac{\eta}{8 \pi} \\
\Pi_{x ; z}^{\tau \bar{\tau}}(\boldsymbol{p}) & =0 \\
\Pi_{y ; z}^{\tau \bar{\tau}}(\boldsymbol{p}) & =0 .
\end{aligned}
$$


${ }_{1210}$ To obtain the interactions in band basis, we define the creation operator $\widetilde{\psi}_{k}^{\dagger}$ which creates a fermion in the upper band, while $\psi_{k}^{\dagger}$ creates 1211 a fermion of definite pseudospin. Changing to the band basis, we use

$$
\begin{aligned}
\psi_{k} & =\mathcal{U}_{k, \tau, s} \widetilde{\psi}_{k} \\
\mathcal{U}_{k, \tau, s} & =\left(\begin{array}{cc}
w_{\tau, s}^{a}(k) & w_{\tau, s}^{a}(k) \\
w_{\tau, s}^{b}(k) e^{i \tau \theta_{k}} & w_{\tau, s}^{b}(k) e^{i \tau \theta_{k}}
\end{array}\right)
\end{aligned}
$$

1212 with the wavefunction components given by, $w_{\tau, s}^{a}(k) \equiv v k / \sqrt{2 \epsilon_{k}\left(\epsilon_{k}-\alpha \tau \eta\right)}, \quad w_{\tau, s}^{b}(k) \equiv\left(\tau \epsilon_{k}-\alpha \eta\right) /(v k) w_{\alpha, k, \tau}^{a}$, with functions ${ }_{1213} w_{\tau, s}^{a}(k), w_{\tau, s}^{b}(k)$ similarly defined for the lower band eigenstates, but not needed.

1214 We then keep only the upper band, e.g. we use the projections

$$
\begin{aligned}
&\left.\mathcal{U}_{[1]}^{\dagger} \sigma_{x} \mathcal{U}_{[2]}\right|_{++}=\left(w_{[1]}^{a} w_{[2]}^{b} e^{i \tau_{2} \theta_{k_{2}}}+w_{[2]}^{a} w_{[1]}^{b} e^{-i \tau_{1} \theta_{k_{1}}}\right), \\
&\left.\mathcal{U}_{[1]}^{\dagger} \sigma_{y} \mathcal{U}_{[2]}\right|_{++}=i\left(-w_{[1]}^{a} w_{[2]}^{b} e^{i \tau_{2} \theta_{k_{2}}}+w_{[2]}^{a} w_{[1]}^{b} e^{-i \tau_{1} \theta_{k_{1}}}\right), \\
&\left.\mathcal{U}_{[1]}^{\dagger} \sigma_{z} \mathcal{U}_{[2]}\right|_{++}=\left(w_{[1]}^{a} w_{[2]}^{a}-w_{[1]}^{b} w_{[2]}^{b} e^{-i \tau_{1} \theta_{k_{1}}+i \tau_{2} \theta_{k_{2}}}\right), \\
&\left.\mathcal{U}_{[1]}^{\dagger} \sigma_{0} \mathcal{U}_{[2]}\right|_{++}=\left(w_{[1]}^{a} w_{[2]}^{a}+w_{[1]}^{b} w_{[2]}^{b} e^{-i \tau_{1} \theta_{k_{1}}+i \tau_{2} \theta_{k_{2}}}\right) .
\end{aligned}
$$

${ }_{1215}$ We compress notation such that indices are $[1]=\left\{k_{1}, \tau_{1}, s_{1}\right\}$. The notation "|++" indicates that we consider just the upper-band 1216 contribution. The phase factors owe to the single particle Berry phase and play a central role in the pairing mechanism.

${ }_{1217}$ In the Cooper channel, $\boldsymbol{k}_{1}=-\boldsymbol{k}_{3}, \boldsymbol{k}_{2}=-\boldsymbol{k}_{4}$, such that $\theta_{k_{3}}=\pi+\theta_{k_{1}}, \theta_{k_{4}}=\pi+\theta_{k_{2}}$. The matrix elements of the screened Coulomb 1218 interaction in the upper band, separated into intravalley and intervalley Cooper channels, are obtained as,

$$
\begin{aligned}
\mathcal{V}_{\text {intra }} & =\left.\sum_{\tau_{i}, s_{i}, \boldsymbol{k}_{i}}\left(\mathcal{U}_{[1]}^{\dagger} \otimes \mathcal{U}_{[3]}^{\dagger} \hat{V}_{I} \mathcal{U}_{[2]} \otimes \mathcal{U}_{[4]}\right)\right|_{++} \delta_{\tau_{1}, \tau_{2}, \tau_{3}, \tau_{4}} \delta_{s_{1}, s_{2}} \delta_{s_{3}, s_{4}} \delta_{\boldsymbol{k}_{1},-\boldsymbol{k}_{3}} \delta_{\boldsymbol{k}_{2},-\boldsymbol{k}_{4}}, \\
\mathcal{V}_{\text {inter }} & =\sum_{\tau_{i}, s_{i}, \boldsymbol{k}_{i}}\left\{\left.\left(\mathcal{U}_{[1]}^{\dagger} \otimes \mathcal{U}_{[3]}^{\dagger} \hat{V}_{I} \mathcal{U}_{[2]} \otimes \mathcal{U}_{[4]}\right)\right|_{++} \delta_{\tau_{1}, \tau_{2},-\tau_{3},-\tau_{4}} \delta_{s_{1}, s_{2}} \delta_{s_{3}, s_{4}} \delta_{\boldsymbol{k}_{1},-\boldsymbol{k}_{3}} \delta_{\boldsymbol{k}_{2},-\boldsymbol{k}_{4}}\right. \\
& \left.+\left.\left(\mathcal{U}_{[1]}^{\dagger} \otimes \mathcal{U}_{[3]}^{\dagger} \hat{V}_{I I} \mathcal{U}_{[2]} \otimes \mathcal{U}_{[4]}\right)\right|_{++} \delta_{\tau_{1},-\tau_{2},-\tau_{3}, \tau_{4}} \delta_{s_{1}, s_{3}} \delta_{s_{2}, s_{4}} \delta_{\boldsymbol{k}_{1},-\boldsymbol{k}_{3}} \delta_{\boldsymbol{k}_{2},-\boldsymbol{k}_{4}}\right\} .
\end{aligned}
$$

${ }_{1219}$ We compactly write this as a spin and valley tensor (pseudpspin has been removed since we work in band basis and keep just the upper 1220 band), using the scattering angle $\theta \equiv \theta_{\boldsymbol{k}_{2}}-\theta_{\boldsymbol{k}_{1}}, \hat{\mathcal{V}}(\theta)=g_{a b c d}(\theta) s_{a} s_{b} \tau_{c} \tau_{d}+j_{a b \alpha \beta}(\theta) s_{a} s_{b} \tau_{\alpha} \tau_{\beta}$, where $a, b, c, d \in\{0, z\}$ and $\alpha, \beta \in \pm$. We 1221 explicitly display the angular dependence of the interaction matrix elements. Considering the angular momentum channel, $l$,

$$
\begin{aligned}
\hat{\mathcal{V}}_{l} & =\int \frac{d \theta}{2 \pi} e^{i l \theta}\left[g_{a b c d} s_{a} s_{b} \tau_{c} \tau_{d}+j_{a b \alpha \beta} s_{a} s_{b} \tau_{\alpha} \tau_{\beta}\right] \\
& =g_{a b c d}^{l} s_{a} s_{b} \tau_{c} \tau_{d}+j_{a b \alpha \beta}^{l} s_{a} s_{b} \tau_{\alpha} \tau_{\beta}
\end{aligned}
$$

${ }_{1222}$ We find that $l= \pm 1$ ( $p$-wave) and $l=0$ ( $s$-wave) are the dominant channels. The resulting tensor is given in equations (12) and (13) in 1223 the main text. 
1225 In order to derive the effective lattice model, we introduce a basis of Wannier orbitals $|\boldsymbol{R}, \alpha\rangle$ localised at the sites $\boldsymbol{R}$ of the honeycomb 1226 lattice, with $\alpha$ being a spin index defined by the action of threefold rotations $\left(C_{3 z}\right)$

$$
u\left(C_{3 z}\right)|\boldsymbol{R}, \alpha\rangle=e^{\frac{2 \pi i}{3} \alpha}|\Lambda \boldsymbol{R}, \alpha\rangle,
$$

${ }_{1227}$ where $\alpha=\left\{ \pm \frac{3}{2}, \pm \frac{1}{2}\right\}$. We consider only the four lowest-energy orbitals on each site.

1228 The lattice Hamiltonian has the form

$$
H=\sum T_{\alpha, \alpha^{\prime}}\left(\boldsymbol{R}, \boldsymbol{R}^{\prime}\right) c_{\boldsymbol{R}, \alpha}^{\dagger} c_{\boldsymbol{R}^{\prime}, \alpha^{\prime}}
$$

1229 where

$$
T_{\alpha, \alpha^{\prime}}\left(\boldsymbol{R}, \boldsymbol{R}^{\prime}\right)=\left\langle\boldsymbol{R}, \alpha\left|H_{2 D H G}\right| \boldsymbol{R}^{\prime}, \alpha^{\prime}\right\rangle
$$

1230 with $H_{2 D H G}$ defined in (1). There is a splitting between the on-site energies $T_{\alpha \alpha}(\boldsymbol{R}, \boldsymbol{R})=\varepsilon_{\alpha}=\varepsilon_{|\alpha|}$ for the $\alpha= \pm \frac{3}{2}$ and $\alpha= \pm \frac{1}{2}$ states, and 1231 we consider an effective model involving only the $\alpha= \pm \frac{3}{2}$ states, which are lowest in energy, and denote $\alpha=\frac{3}{2} s$ where $s$ is the spin index 1232 used throughout the main text, and $c_{\boldsymbol{R}, \alpha} \rightarrow c_{\boldsymbol{R}, s}$.

${ }_{1233}$ The topological mass term originates from nearest neighbour hopping terms which involve a spin transition $\alpha^{\prime}-\alpha= \pm 2$. By symmetry 1234 we find, for hopping from a site $\boldsymbol{R}$ to a nearest neighbour $\boldsymbol{R}+\boldsymbol{d}$,

$$
T_{\mp \frac{1}{2}, \pm \frac{3}{2}}(\boldsymbol{R}+\boldsymbol{d}, \boldsymbol{R})=\lambda d_{ \pm}^{2}
$$

1235 An effective spin-conserving next nearest neighbour hopping term arises due to two consecutive hoppings with initial, intermediate and final 1236 sites $\boldsymbol{R}, \boldsymbol{R}+\boldsymbol{d}$, and $\boldsymbol{R}+\boldsymbol{d}+\boldsymbol{d}^{\prime}$ respectively

$$
T_{s s}^{\mathrm{eff}}\left(\boldsymbol{R}+\boldsymbol{d}+\boldsymbol{d}^{\prime}, \boldsymbol{R}\right)=\frac{\lambda^{2}}{\varepsilon_{\frac{3}{2}}-\varepsilon_{\frac{1}{2}}}|\boldsymbol{d}|^{2} e^{2 i s \sigma\left(\theta^{\prime}-\theta\right)}
$$

${ }_{1237}$ where $\theta, \theta^{\prime}$ are the hopping directions in the first and second steps respectively and $\sigma=+1,-1$ when $\boldsymbol{R} \in A, B$ respectively. ${ }_{1238}$ Choosing lattice vectors $\boldsymbol{a}_{1}=(a, 0), \boldsymbol{a}_{2}=(a / 2, a \sqrt{3} / 2)$, and denoting the three nearest neighbour bonds $\boldsymbol{d}_{i}=\boldsymbol{R}-\boldsymbol{R}^{\prime}$ with $\boldsymbol{R}^{\prime}$ in the ${ }_{1239}$ A sublattice and $\boldsymbol{R}$ a neighbouring site, and the six next nearest neighbour bonds $\widetilde{\boldsymbol{d}}_{n}$ which are vectors of length $a$ directed along angles ${ }_{1240} \theta_{n}=\frac{n \pi}{3}$ for $n=\{0,1,2,3,4,5\}$, we obtain an effective Hamiltonian involving only the $|\alpha|=\frac{3}{2}$ states (after absorbing the on-site potential 1241 into the chemical potential)

$$
H=-t \sum_{\left\langle\boldsymbol{R}+\boldsymbol{d}_{i}, \boldsymbol{R}\right\rangle} c_{\boldsymbol{R}+\boldsymbol{d}_{i}, s}^{\dagger} c_{\boldsymbol{R}, s}-t^{\prime} \sum_{\left\langle\left\langle\boldsymbol{R}+\widetilde{\boldsymbol{d}}_{n}, \boldsymbol{R}\right\rangle\right\rangle} e^{i s \sigma \varphi_{n}} c_{\boldsymbol{R}+\widetilde{\boldsymbol{d}}_{n}, s}^{\dagger} c_{\boldsymbol{R}, s}
$$

1242 where $\varphi_{n}=\frac{2 \pi}{3}$ for $n=0,2,4$ and $\varphi_{n}=-\frac{2 \pi}{3}$ for $n=1,3,5$.

${ }_{1243}$ We make contact between the two forms of the normal state Hamiltonian by expanding the Hamiltonian near the $K$ points, and reproduce 1244 the effective Dirac Hamiltonian

$$
\mathcal{H}(\tau \boldsymbol{K}+\boldsymbol{k}) \approx v\left(\tau k_{x} \sigma_{x}+k_{y} \sigma_{y}\right)+\eta \tau \sigma_{z} s_{z}
$$

1245 where we find the relation between the parameters in the Dirac theory and in the real space model

$$
v=\frac{\sqrt{3} a t}{2}, \quad \eta=\frac{9}{2} t^{\prime}
$$


${ }_{1246}$ Near the $K$ points we have the upper band eigenstates

$$
\widetilde{\psi}_{\boldsymbol{k} \tau s}^{\dagger}=\sum_{\boldsymbol{R}} \varphi_{\boldsymbol{k} \tau s}(\boldsymbol{R}) c_{\boldsymbol{R} s}^{\dagger}
$$

1247 with symmetry properties $\varphi_{\boldsymbol{k} \tau s}(-\boldsymbol{R})=\varphi_{-\boldsymbol{k} \bar{\tau} s}(\boldsymbol{R})$ and $\varphi_{-\boldsymbol{k} \bar{\tau} \downarrow}(\boldsymbol{R})=\varphi_{\boldsymbol{k} \tau \uparrow}^{*}(\boldsymbol{R})$. Explicitly,

$$
\varphi_{\boldsymbol{k} \tau s}(\boldsymbol{R})=\frac{1}{\sqrt{2}} e^{i(\tau \boldsymbol{K}+\boldsymbol{k}) \cdot \boldsymbol{R}}\left(w_{\tau, s}^{a}(k) a(\boldsymbol{R})+e^{i \tau \theta_{\boldsymbol{k}}} w_{\tau, s}^{a}(k) b(\boldsymbol{R})\right)
$$

1248 with $a(\boldsymbol{R})=\{1,0\}, b(\boldsymbol{R})=\{0,1\}$, for $\boldsymbol{R} \in A, B$ respectively, and the functions $w_{\tau, s}^{a}(k)$ and $w_{\tau, s}^{b}(k)$ are defined in Section C. We shall use 1249 these wavefunctions to obtain a real space form for the the superconducting gap functions we have written in momentum space in Section $1250 \mathrm{~V}$, 
The mean field BdG Hamiltonian is

$$
H=\sum_{\boldsymbol{k}, \tau, s} \varepsilon_{\boldsymbol{k}} \widetilde{\psi}_{\boldsymbol{k} \tau s}^{\dagger} \widetilde{\psi}_{\boldsymbol{k} \tau s}+\frac{1}{2} \sum_{\boldsymbol{k},-\boldsymbol{k}, \tau, \tau^{\prime}, s, s^{\prime}} \widetilde{\psi}_{\boldsymbol{k} \tau s}^{\dagger}\left(\Delta_{\boldsymbol{k}}\right)_{\tau s, \tau^{\prime} s^{\prime}} \widetilde{\psi}_{-\boldsymbol{k} \tau^{\prime}, s^{\prime}}^{\dagger}+\text { h.c. }
$$

${ }_{1253}$ where we have used $\psi_{\boldsymbol{k} \tau s}$ to refer to the upper band creation operator, as in the previous subsection. The three superconducting phases we 1254 study are given by

$$
\Delta_{\boldsymbol{k}}=\Delta_{k} \times\left\{\begin{array}{l}
d_{s}^{z} s_{z} \tau_{0}\left(\tau_{y} s_{y}\right) \\
e^{ \pm i \theta_{\boldsymbol{k}}} d_{s}^{z} s_{z} \tau_{z}\left(\tau_{y} s_{y}\right) \\
e^{i \tau_{z}\left(\phi-\theta_{\boldsymbol{k}}\right)}\left(d_{s}^{x} s_{x}+d_{s}^{y} s_{y}\right) \tau_{y}\left(\tau_{y} s_{y}\right)
\end{array}\right.
$$

1255 for the $s_{\tau}, p+i p$ and $p+i \tau p$ phases respectively. We have added a factor $\Delta_{k}$ absent in the main text. This is a smooth function peaked at 1256 the Fermi momentum, encapsulating the fact that pairing should only occur near the Fermi surface, and should be retained in deriving the ${ }_{1257}$ correct real space gap function. Writing

$$
\Delta_{\boldsymbol{k} ; \tau \tau^{\prime} ; s s^{\prime}}=\Delta_{\tau \tau^{\prime}}(\boldsymbol{k})\left(d^{\mu} s_{\mu} i \hat{s}_{y}\right)_{s s^{\prime}}
$$

1258 to separate out the spin structure, we can use the upper band wavefunctions to go to the coordinate representation in terms of the full real 1259 space creation operator $c_{\boldsymbol{r} s}^{\dagger}$,

$$
H=\frac{1}{2} \sum\left(d^{\mu} s_{\mu} i \hat{s}_{y}\right)_{s s^{\prime}} \varphi_{\boldsymbol{k} \tau s}(\boldsymbol{R}) \Delta_{\tau \tau^{\prime}}(\boldsymbol{k}) \varphi_{-\boldsymbol{k} \tau^{\prime} s^{\prime}}\left(\boldsymbol{R}^{\prime}\right) c_{\boldsymbol{R} s}^{\dagger} c_{\boldsymbol{R}^{\prime} s^{\prime}}^{\dagger}=\frac{1}{2} \sum \Delta_{s s^{\prime}}\left(\boldsymbol{R}, \boldsymbol{R}^{\prime}\right) c_{\boldsymbol{R} s}^{\dagger} c_{\boldsymbol{R}^{\prime} s^{\prime}}^{\dagger}
$$

1260 Note that under inversion, $\boldsymbol{R} \rightarrow-\boldsymbol{R}, \boldsymbol{R}^{\prime} \rightarrow-\boldsymbol{R}^{\prime}$ we have

$$
\begin{aligned}
\Delta_{s s^{\prime}}\left(-\boldsymbol{R},-\boldsymbol{R}^{\prime}\right) & =\sum_{\boldsymbol{k}}\left(d^{\mu} \hat{s}_{\mu} i \hat{s}_{y}\right)_{s s^{\prime}} \varphi_{-\boldsymbol{k} \bar{\tau} s}(\boldsymbol{R}) \Delta_{\tau \tau^{\prime}}(\boldsymbol{k}) \varphi_{\boldsymbol{k} \bar{\tau}^{\prime} s^{\prime}}\left(\boldsymbol{R}^{\prime}\right) \\
& =\sum_{\boldsymbol{k}}\left(d^{\mu} \hat{s}_{\mu} i \hat{s}_{y}\right)_{s s^{\prime}} \varphi_{\boldsymbol{k} \tau s}(\boldsymbol{R}) \Delta_{\bar{\tau} \bar{\tau}^{\prime}}(-\boldsymbol{k}) \varphi_{-\boldsymbol{k} \tau^{\prime} s}(\boldsymbol{R})
\end{aligned}
$$

1261 and the valley structures are explicitly given by

$$
\Delta_{\tau \tau^{\prime}}(\boldsymbol{k})=\Delta_{k} \times\left\{\begin{array}{l}
\left(i \hat{\tau}_{y}\right)_{\tau \tau^{\prime}} \\
e^{ \pm i \theta_{\boldsymbol{k}}}\left(\hat{\tau}_{z} i \hat{\tau}_{y}\right)_{\tau \tau^{\prime}} \\
e^{i \hat{\tau}_{z}\left(\phi-\theta_{\boldsymbol{k}}\right)}
\end{array}\right.
$$

We can now explicitly evaluate the functions $\Delta\left(\boldsymbol{R}, \boldsymbol{R}^{\prime}\right)$. We begin with the intervalley phases, which take the form

$$
H_{\Delta}=\sum_{\boldsymbol{k} ; s} \Delta_{k} e^{i \ell \theta_{\boldsymbol{k}}} \widetilde{\psi}_{\boldsymbol{k}+s}^{\dagger} \widetilde{\psi}_{-\boldsymbol{k}-\bar{s}}^{\dagger}
$$

${ }_{1263}$ where $\ell=0$ for the $s_{ \pm}$phase and $\ell= \pm 1$ for the $p \pm i p$ phases. Expanding $\widetilde{\psi}_{\boldsymbol{k} \tau s}^{\dagger}$ in the position basis (D9) we find

$$
H_{\Delta}=\sum \Delta_{k} e^{i \ell \theta_{\boldsymbol{k}}} \varphi_{\boldsymbol{k}+s}(\boldsymbol{R}) \varphi_{-\boldsymbol{k}-\bar{s}}\left(\boldsymbol{R}^{\prime}\right) c_{\boldsymbol{R} s}^{\dagger} c_{\boldsymbol{R}^{\prime} \bar{s}}^{\dagger}=\sum \Delta\left(\boldsymbol{R}, \boldsymbol{R}^{\prime}\right) c_{\boldsymbol{R}_{\uparrow}}^{\dagger} c_{\boldsymbol{R}^{\prime} \downarrow}^{\dagger}
$$

1264 where we may write

$$
\Delta\left(\boldsymbol{R}, \boldsymbol{R}^{\prime}\right)=\sum_{\boldsymbol{k}} \Delta_{k} e^{i \ell \theta_{\boldsymbol{k}}}\left[\varphi_{\boldsymbol{k}+\uparrow}(\boldsymbol{R}) \varphi_{\boldsymbol{k}+\uparrow}^{*}\left(\boldsymbol{R}^{\prime}\right)-\varphi_{\boldsymbol{k}+\uparrow}(-\boldsymbol{R}) \varphi_{\boldsymbol{k}+\uparrow}^{*}\left(-\boldsymbol{R}^{\prime}\right)\right]
$$


${ }_{1265}$ In order to perform the summation over $k$ we introduce the functions $f_{m}^{\sigma \sigma^{\prime}}(l)$ defined by

$$
f_{m}^{\sigma \sigma^{\prime}}(l)=\int \Delta_{k} w_{++}^{\sigma}(k) w_{++}^{\sigma^{\prime}}(k) J_{m}(k l) \frac{k d k}{2 \pi}
$$

1266 with $m=0,1,2, \ldots$, and the relation

$$
\sum w_{++}^{\sigma}(k) w_{++}^{\sigma^{\prime}}(k) \Delta_{k} e^{i\left(\boldsymbol{k} \cdot\left(\boldsymbol{R}-\boldsymbol{R}^{\prime}\right)+\ell \theta_{\boldsymbol{k}}\right)}=i^{|\ell|} e^{i \ell \theta} f_{|\ell|}^{\sigma \sigma^{\prime}}\left(\left|\boldsymbol{R}-\boldsymbol{R}^{\prime}\right|\right),
$$

${ }_{1267}$ where $\theta=\theta_{\boldsymbol{R}}-\theta_{\boldsymbol{R}^{\prime}}$.

${ }_{1268}$ The function $f_{0}^{\sigma \sigma^{\prime}}(l)$ is peaked at $l=0$ and oscillates over length scales $\sim k_{F}^{-1}$ with decaying amplitude, while for $m>0$ the functions ${ }_{1269} f_{m}^{\sigma \sigma^{\prime}}(l)$ vanish at $l=0$, increase to a global maximum at $l \approx k_{F}^{-1}$ and then decays for larger values of $l$.

${ }_{1270}$ In terms of the functions $f_{m}^{\sigma \sigma}(l)$ the gap $\Delta\left(\boldsymbol{R}, \boldsymbol{R}^{\prime}\right)$ is given by

$$
\begin{aligned}
& \Delta\left(\boldsymbol{R}, \boldsymbol{R}^{\prime}\right)
\end{aligned}
$$

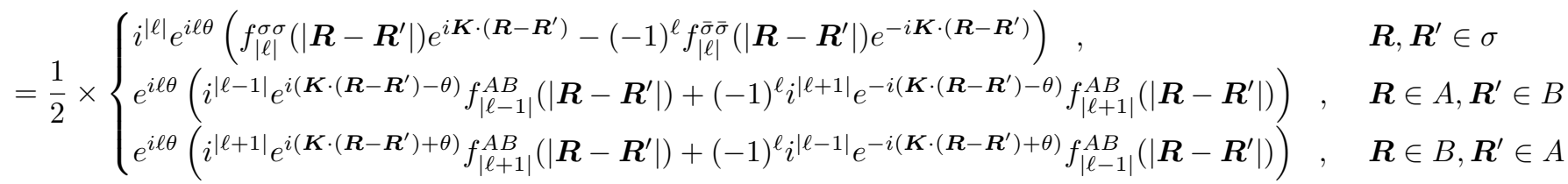

${ }_{1271}$ Note that for $\boldsymbol{R}, \boldsymbol{R}^{\prime} \in \sigma$ we have

$$
\Delta\left(\boldsymbol{R}, \boldsymbol{R}^{\prime}\right)=-\frac{1}{2} i^{|\ell|} e^{i \ell \theta}\left(f_{|\ell|}^{\bar{\sigma} \bar{\sigma}}\left(\left|\boldsymbol{R}-\boldsymbol{R}^{\prime}\right|\right) e^{i \boldsymbol{K} \cdot\left(\boldsymbol{R}-\boldsymbol{R}^{\prime}\right)}-(-1)^{\ell} f_{|\ell|}^{\sigma \sigma}\left(\left|\boldsymbol{R}-\boldsymbol{R}^{\prime}\right|\right) e^{-i \boldsymbol{K} \cdot\left(\boldsymbol{R}-\boldsymbol{R}^{\prime}\right)}\right)
$$

1272 while for $\boldsymbol{R}^{\prime} \in A, \boldsymbol{R} \in B$ we have

$$
\Delta\left(\boldsymbol{R}, \boldsymbol{R}^{\prime}\right)=\frac{1}{2}\left(i^{|\ell+1|} e^{i\left(\boldsymbol{K} \cdot\left(\boldsymbol{R}-\boldsymbol{R}^{\prime}\right)+(\ell+1) \theta_{\left|\boldsymbol{R}-\boldsymbol{R}^{\prime}\right|}\right)} f_{|\ell+1|}^{A B}\left(\left|\boldsymbol{R}-\boldsymbol{R}^{\prime}\right|\right)+(-1)^{\ell} i^{|\ell-1|} e^{i\left(-\boldsymbol{K} \cdot\left(\boldsymbol{R}-\boldsymbol{R}^{\prime}\right)+(\ell-1) \theta\right)} f_{|\ell-1|}^{A B}\left(\left|\boldsymbol{R}-\boldsymbol{R}^{\prime}\right|\right)\right)
$$

${ }_{1273}$ For nearest neighbours, $\boldsymbol{R}-\boldsymbol{R}^{\prime}=\boldsymbol{d}_{i}$, recall that we have $\boldsymbol{K} \cdot \boldsymbol{d}_{i}=\{0,-2 \pi / 3,2 \pi / 3\}$ and $\theta_{\boldsymbol{R}}=\theta_{i}=\{\pi / 2, \pi / 2+2 \pi / 3, \pi / 2+4 \pi / 3\}$, giving ${ }_{1274}$ us $\boldsymbol{K} \cdot \boldsymbol{R}+\theta_{\boldsymbol{R}}=\frac{\pi}{2}$. Thus for $\boldsymbol{R}^{\prime} \in A$ we have

$$
\Delta\left(\boldsymbol{R}^{\prime}+\boldsymbol{d}_{i}, \boldsymbol{R}^{\prime}\right)=\frac{1}{2} e^{i \ell \theta_{i}}\left(i^{|\ell+1|+1} f_{|\ell+1|}^{A B}\left(\frac{a}{\sqrt{3}}\right)+(-1)^{\ell} i^{|\ell-1|-1} f_{|\ell-1|}^{A B}\left(\frac{a}{\sqrt{3}}\right)\right)
$$

${ }_{1275}$ For next nearest neighbours, $\boldsymbol{R}-\boldsymbol{R}^{\prime}=\widetilde{\boldsymbol{d}}_{n}^{\prime}$ we have

$$
e^{i \boldsymbol{K} \cdot\left(\boldsymbol{R}-\boldsymbol{R}^{\prime}\right)}= \begin{cases}e^{-\frac{2 \pi i}{3}}, & i=1,3,5 \\ e^{\frac{2 \pi i}{3}}, & i=2,4,6\end{cases}
$$

1276 which gives us, for $\boldsymbol{R}-\boldsymbol{R}^{\prime}=\widetilde{\boldsymbol{d}}_{n}$

$$
\Delta\left(\boldsymbol{R}, \boldsymbol{R}^{\prime}\right)= \begin{cases}\frac{1}{2} i^{|\ell|} e^{i \ell \theta_{i}^{\prime}}\left(f_{|\ell|}^{\sigma \sigma}(a) e^{-\frac{2 \pi i}{3}}-(-1)^{\ell} f_{|\ell|}^{\bar{\sigma} \bar{\sigma}}(a) e^{\frac{2 \pi i}{3}}\right), \quad i=1,3,5 \\ \frac{1}{2} i^{|\ell|} e^{i \ell \theta_{i}^{\prime}}\left(f_{|\ell|}^{\sigma \sigma}(a) e^{\frac{2 \pi i}{3}}-(-1)^{\ell} f_{|\ell|}^{\bar{\sigma} \bar{\sigma}}(a) e^{-\frac{2 \pi i}{3}}\right), \quad i=2,4,6\end{cases}
$$

${ }_{1277}$ with $\theta_{i}^{\prime}$ being the angle between $\boldsymbol{d}_{i}^{\prime}$ and the $x$ axis.

1278 a. $s_{\tau}$ 
1280 and therefore restrict the pairing to next nearest neighbours only. The gap depends on the function $f_{0}^{\sigma \sigma}(a)$. Writing $f_{0}^{A A}(a)=\alpha+\beta$, ${ }_{1281} f_{0}^{B B}(a)=\alpha-\beta$ we have from (E16)

$$
\begin{aligned}
\Delta\left(\boldsymbol{R}, \boldsymbol{R}^{\prime}\right)= & \begin{cases}\frac{1}{2}\left((\alpha+\beta) e^{-\frac{2 \pi i}{3}}-(\alpha-\beta) e^{\frac{2 \pi i}{3}}\right), & i=1,3,5 \\
\frac{1}{2}\left((\alpha+\beta) e^{\frac{2 \pi i}{3}}-(\alpha-\beta) e^{-\frac{2 \pi i}{3}}\right), & i=2,4,6\end{cases} \\
& = \begin{cases}-\frac{1}{2}(\beta+i \sqrt{3} \alpha), & i=1,3,5 \\
-\frac{1}{2}(\beta-i \sqrt{3} \alpha), & i=2,4,6\end{cases}
\end{aligned}
$$

${ }_{1282}$ In the limit of spin-orbit interaction we have $\beta \rightarrow 0$. For the numerical diagonalisation we choose a gap in which $\beta=0$ (since the spin-orbit 1283 interaction is weak) and $\alpha=i \Delta^{\prime}$, so that $\Delta\left(\boldsymbol{R}, \boldsymbol{R}^{\prime}\right)$ is purely real.

${ }_{1285}$ For exact diagonalisation we take only the nearest neighbour pairing terms. For $\boldsymbol{R}^{\prime} \in A, \boldsymbol{R}=\boldsymbol{R}^{\prime}+\boldsymbol{d}_{i}$, the gap is given by setting $\ell=+1$ 1286 in (E15),

$$
\begin{gathered}
\Delta\left(\boldsymbol{R}, \boldsymbol{R}^{\prime}\right)=\frac{1}{2} e^{i \theta}\left[-i f_{2}^{A B}(R)+i f_{0}^{A B}(R)\right] \\
=\Delta^{\prime} e^{i \theta}
\end{gathered}
$$

${ }_{1287}$ where $\theta=\theta_{\boldsymbol{R}}-\theta_{\boldsymbol{R}^{\prime}}$.

${ }_{1289}$ We now consider the $p+i \tau p$ phase. Since the $\boldsymbol{d}_{s}$ vector is pinned in-plane for this phase, pairing is between the same spin species, i.e. 1290 the gap is proportional to $s_{z}$. This way, BdG Hamiltonian can be decomposed into spin blocks, the Majoranas corner states associated to 1291 each of which are related by time-reversal symmetry.

${ }_{1292}$ The derivation then proceeds through more or less the same manipulations as above (c.f. Appendix A4 of [1]). It is possible to decompose ${ }_{1293}$ the pairing term into two identical spin blocks,

$$
H_{\Delta}=\frac{1}{2} \sum_{k, \tau, s} \Delta_{k} e^{i \tau \phi} e^{-i \tau \theta_{\boldsymbol{k}}} \widetilde{\psi}_{\boldsymbol{k}, \tau, s}^{\dagger} \widetilde{\psi}_{-\boldsymbol{k}, \tau, s}^{\dagger}=\frac{1}{2} \sum_{s} \Delta\left(\boldsymbol{R}, \boldsymbol{R}^{\prime}\right) c_{\boldsymbol{R}, s}^{\dagger} c_{\boldsymbol{R}^{\prime}, s}^{\dagger} .
$$

Expanding $\widetilde{\psi}_{\boldsymbol{k}, \tau, s}$ in the position basis (D9) we find

$$
\begin{gathered}
\Delta\left(\boldsymbol{R}, \boldsymbol{R}^{\prime}\right)=\sum_{\boldsymbol{k}} \Delta_{k}\left\{\frac{1}{2} e^{i\left\{\boldsymbol{K} \cdot\left(\boldsymbol{R}+\boldsymbol{R}^{\prime}\right)+\boldsymbol{k} \cdot\left(\boldsymbol{R}-\boldsymbol{R}^{\prime}\right)+\phi-\theta_{\boldsymbol{k}}\right\}}\left[\left(w_{+, s}^{a}(k) a(\boldsymbol{R})+e^{i \theta_{\boldsymbol{k}}} w_{+, s}^{b}(k) b(\boldsymbol{R})\right)\left(w_{+, s}^{a}(k) a\left(\boldsymbol{R}^{\prime}\right)-e^{i \theta_{\boldsymbol{k}}} w_{+, s}^{b}(k) b\left(\boldsymbol{R}^{\prime}\right)\right)\right]\right. \\
\left.+\frac{1}{2} e^{i\left\{-\boldsymbol{K} \cdot\left(\boldsymbol{R}+\boldsymbol{R}^{\prime}\right)+\boldsymbol{k} \cdot\left(\boldsymbol{R}-\boldsymbol{R}^{\prime}\right)-\phi+\theta_{\boldsymbol{k}}\right\}}\left[\left(w_{-, s}^{a}(k) a(\boldsymbol{R})+e^{-i \theta_{\boldsymbol{k}}} w_{-, s}^{b}(k) b(\boldsymbol{R})\right)\left(w_{-, s}^{a}(k) a\left(\boldsymbol{R}^{\prime}\right)-e^{-i \theta_{\boldsymbol{k}}} w_{-, s}^{b}(k) b\left(\boldsymbol{R}^{\prime}\right)\right)\right]\right\} .
\end{gathered}
$$

${ }_{1295}$ Performing the summation over $\boldsymbol{k}$ yields functions $f_{m}^{\sigma \sigma^{\prime}}\left(\left|\boldsymbol{R}-\boldsymbol{R}^{\prime}\right|\right)$ which all vanish at small separations $\boldsymbol{R}-\boldsymbol{R}^{\prime} \ll k_{F}^{-1}$ except for $m=0$. 1296 For purposes of exact diagonalisation, we keep only terms involving nearest neighbours, which correspond to those that cancel the winding ${ }_{1297}$ factor $e^{i \theta_{k}}$. This gives

$$
\begin{gathered}
\Delta\left(\boldsymbol{R}, \boldsymbol{R}^{\prime}\right)=\sum_{\boldsymbol{k}} \Delta_{k}\left\{\frac{1}{2} e^{i\left\{\boldsymbol{K} \cdot\left(\boldsymbol{R}+\boldsymbol{R}^{\prime}\right)+\boldsymbol{k} \cdot\left(\boldsymbol{R}-\boldsymbol{R}^{\prime}\right)+\phi-\theta_{\boldsymbol{k}}\right\}} w_{+, s}^{a} w_{+, s}^{b} e^{i \theta_{\boldsymbol{k}}}\left(-a(\boldsymbol{R}) b\left(\boldsymbol{R}^{\prime}\right)+b(\boldsymbol{R}) a\left(\boldsymbol{R}^{\prime}\right)\right)\right. \\
\left.+\frac{1}{2} e^{i\left\{-\boldsymbol{K} \cdot\left(\boldsymbol{R}+\boldsymbol{R}^{\prime}\right)+\boldsymbol{k} \cdot\left(\boldsymbol{R}-\boldsymbol{R}^{\prime}\right)-\phi+\theta_{\boldsymbol{k}}\right\}} w_{-, s}^{a} w_{-, s}^{b} e^{-i \theta_{\boldsymbol{k}}}\left(-a(\boldsymbol{R}) b\left(\boldsymbol{R}^{\prime}\right)+b(\boldsymbol{r}) a\left(\boldsymbol{R}^{\prime}\right)\right)\right\}
\end{gathered}
$$


${ }_{1298}$ We note that $w_{-, s}^{a} w_{-, s}^{b}=-w_{+, s}^{a} w_{+, s}^{b}$, and is independent of spin index $s$. Performing the summation over $\boldsymbol{k}$

$$
\int w_{+, s}^{a} w_{+, s}^{b} \Delta_{k} e^{i \boldsymbol{k} \cdot\left(\boldsymbol{R}-\boldsymbol{R}^{\prime}\right)} \frac{d^{2} \boldsymbol{k}}{(2 \pi)^{2}}=\int \frac{v k}{\varepsilon_{\boldsymbol{k}}} \Delta_{k} J_{0}\left(k\left|\boldsymbol{R}-\boldsymbol{R}^{\prime}\right|\right) \frac{k d k}{2 \pi}=f_{0}^{A B}\left(\left|\boldsymbol{R}-\boldsymbol{R}^{\prime}\right|\right)
$$

1299 we find

$$
\begin{aligned}
\Delta\left(\boldsymbol{R}, \boldsymbol{R}^{\prime}\right)= & \frac{1}{2} f_{0}^{A B}\left(\left|\boldsymbol{R}-\boldsymbol{R}^{\prime}\right|\right)\left[e^{i\left\{\boldsymbol{K} \cdot\left(\boldsymbol{R}+\boldsymbol{R}^{\prime}\right)+\phi\right\}}-e^{i\left\{-\boldsymbol{K} \cdot\left(\boldsymbol{R}+\boldsymbol{R}^{\prime}\right)-\phi\right\}}\right]\left[-a(\boldsymbol{R}) b\left(\boldsymbol{R}^{\prime}\right)+b(\boldsymbol{R}) a\left(\boldsymbol{R}^{\prime}\right)\right] \\
& =i f_{0}^{A B}\left(\left|\boldsymbol{R}-\boldsymbol{R}^{\prime}\right|\right)\left[\sin \left(\boldsymbol{K} \cdot\left(\boldsymbol{R}+\boldsymbol{R}^{\prime}\right)+\phi\right)\right]\left[-a(\boldsymbol{R}) b\left(\boldsymbol{R}^{\prime}\right)+b(\boldsymbol{R}) a\left(\boldsymbol{R}^{\prime}\right)\right]
\end{aligned}
$$

1300 and therefore, with $\boldsymbol{R} \in A, \boldsymbol{R}^{\prime} \in B$,

$$
H_{\Delta}=\sum_{\left\langle\boldsymbol{R}, \boldsymbol{R}^{\prime}\right\rangle} \Delta^{\prime}\left[\sin \left(\boldsymbol{K} \cdot\left(\boldsymbol{R}+\boldsymbol{R}^{\prime}\right)+\phi\right) c_{\boldsymbol{R}^{\dagger}}^{c_{\boldsymbol{R}^{\prime}}^{\dagger}}+\text { h.c. }\right]
$$

1301 where $\Delta^{\prime}=i f_{0}^{A B}\left(\left|\boldsymbol{R}-\boldsymbol{R}^{\prime}\right|\right)$. 